\title{
VI. LES TRADUCTIONS FRANÇAISES DES LOIS FONDAMENTALES DU SAINT-EMPIRE
}

\section{Les traductions françaises des traités de Westphalie (1648)}

Le Saint Empire romain germanique n'avait pas de Constitution écrite unique. Parmi les sources les plus importantes qui ont régi son droit d'État, on peut cependant nommer un certain nombre de lois fondamentales ${ }^{1}$, telles que la Bulle d'or de 1356, la paix publique de l'Empire de 1495 , les capitulations impériales jurées par les empereurs et les rois des Romains depuis 1519, la paix de religion d'Augsbourg de 1555 et, surtout, la paix de Westphalie de 1648, qui passe pour sa loi fondamentale la plus importante jusqu'en $1806^{2}$. Les questions politiques et constitutionnelles faisaient surtout, mais non exclusivement, l'objet de l'article VIII de l'»Instrumentum Pacis Osnabrugense ${ }^{3}$. Quand, à la mi-novembre, la »Gazette de France « publia la nouvelle de la signature des traités de Westphalie ${ }^{4}$ (qu'on appelait alors, au singulier, le »traité d'Allemagne «), aucun mot ne fut prononcé sur la cession de l'Alsace ni sur le fait que les affaires d'Allemagne et d'Italie avaient été réglées d'une manière assez avantageuse pour la France. C'est à ce moment-là la guerre contre l'Espagne qui l'emporte par rapport à tous les autres tourments sur l'échiquier international ${ }^{5}$, et le souci de voir les Frondeurs encouragés par la persistance de ce défi extérieur. D'où l'insistance sur le paragraphe 3 du traité de Münster, qui neutralisait le cercle de Bourgogne et interdisait aux parties contractantes d'assister les ennemis de l'une comme de l'autre. C'est la seule clause particulière des traités à laquelle fait allusion ce numéro de la »Gazette de France«,

\footnotetext{
1 Au sujet des lois fondamentales de la monarchie française, il est toujours utile de consulter LEMAIRE, Les lois fondamentales.

2 Il n'y avait pas de catalogue fixe des lois fondamentales du Saint Empire romain germanique; un très grand nombre de juristes mettaient aussi le traité de Passau (1552) au nombre des lois constitutionnelles. C'est pourquoi nous évoquerons aussi ses versions françaises.

${ }^{3}$ Art. VIII du traité d'Osnabrück (Instrumentum Pacis Osnabrugense, IPO) $=\$ \S 62-66$ du traité de Münster (Instrumentum pacis Monasteriense, IPM); texte latin: OsCHMANN, APW III B $1 / 1$, p. 19-21 et 130-131.

${ }^{4}$ Gazette [de France], ici Recueil des nouvelles ordinaires et extraordinaires, relations et recits des choses avenues, tant en ce royaume qu'ailleurs, pendant l'année mil six cent quarante-huit, Paris, Du Bureau d'Adresse, aux Galleries du Louvre, devant la rüe Saint Thomas, $1649, \mathrm{n}^{\circ} 171$ du 13 novembre 1648 (les dates de publication sont toujours indiquées selon l'édition parisienne), intitulé: La publication de la paix generale d'entre l'Empire et la France, dans les villes de Munster $\&$ d'Osnabruck, en suite de sa signature, $\&$ ce qui s'y est passé [...], p. 1517-1528. Nous utilisons le terme »Gazette de France « bien qu'il date de 1762 ; en 1648, on disait "Gazette « mais cette dénomination est devenue ambiguë.

5 Au sujet des négociations franco-espagnoles en Westphalie, considérées comme primordiales par la délégation française, mais interrompues sans résultats définitifs en 1648, voir notamment Tischer, Französische Diplomatie, p. 321-410; ROHRSCHNEIDER, Der gescheiterte Frieden; SÉRÉ, La paix des Pyrénées, p. 119-166.
} 
qui devait pourtant publier par la suite des abrégés assez précis des deux traités 6 . Les années 1648-1650 sont marquées par la parution d'un nombre important de résumés des principales stipulations de paix, qui traduisent la volonté du pouvoir politique de les exploiter à des fins de propagande. La publication de traités de paix n'échappe pas à l'évolution générale que subit l'édition française au cours du XVIIe siècle. En France, la Fronde est l'époque où l'imprimé se transforme largement en instrument de propagande anti- ou pro-gouvernementale ${ }^{7}$. Les exploits d'une politique étrangère heureuse, qui avaient été consacrés par les traités de Westphalie, constituaient alors un sujet fécond dont la propagande royale et gouvernementale cherchait à tirer profit en favorisant la publication de toute une série d'écrits littéraires et non littéraires qui devaient influencer l'opinion publique dans le sens du gouvernement. Il faut pourtant nuancer le tableau dessiné ci-dessus et ne pas chercher partout une politique systématique et réfléchie de propagande: le pouvoir n'a souvent fait que réagir face à la demande d'un éditeur de lui accorder la permission de faire imprimer tel ou tel livre, tel ou tel document officiel, qu'on jugeait favorable à la politique du gouvernement et dont on favorisait par conséquent la publication. Il en va ainsi pour un certain nombre d'éditions des traités de Westphalie. Les premières d'entre elles, qui parurent entre 1648 et 1650, témoignent pourtant, par certains aspects, d'une politique gouvernementale plus active dans ce domaine.

\subsection{Les premières traductions de 1648 à 1650}

Certes, l'Ancien Régime ne disposait pas d'un journal officiel stricto sensu tel que nous le connaissons aujourd'hui. Cependant, certaines éditions de sommaires des traités de Münster et d'Osnabrück montrent des particularités qui font qu'ils ressemblent, soit physiquement, soit par leur lieu d'impression ou par leur éditeur, à une publication sinon officielle, du moins officieuse. Cela vaut aussi pour leur publication dans la »Gazette de France ${ }^{8}$. Celle-ci représente, parmi les périodiques

${ }^{6}$ Le sujet de l'interdiction de l'assistance de l'Espagne par l'empereur sera d'ailleurs repris dans le commentaire que la »Gazette« ajouta à la publication du résumé du traité de Münster (voir ci-dessous).

7 Cf. notamment Christian Jouhaud, Mazarinades: la fronde des mots, Paris 1985; Hubert CARRIER, Les Mazarinades. [Avant-titre:] La presse de la Fronde (1648-1653), 2 vol., Genève 1989-1991 (École pratique des hautes études, IVe section: Sciences historiques et philologiques. VI. Histoire et civilisation du livre, 19-20); Robert DAMIEN, Bibliothèque et Etat. Naissance d'une raison politique dans la France du XVIIe siècle, Paris 1995; Minors, Censure et culture. Labarre souligne, à côté de la Fronde, deux autres périodes de pointe de la publication d'occasionnels et de feuilles volantes à fin de propagande: le temps de la Ligue et la minorité de Louis XIII, Albert LaBarre, Histoire du livre, Paris ${ }^{2} 1974$, p. 97.

8 Notons que, au début du XXe siècle, Granges de Surgères n'hésitait pas à appeler la »Gazette« un »Journal Officiel « de l'Ancien Régime, Marquis DE GRANGES DE SURGĖRES, Répertoire historique et biographique de la Gazette de France depuis l'origine jusqu'à la Révolution. 1631-1790, 4 vol., Paris 1902-1906, ici t. I, p. VII. Il est aussi à noter que Chartier va jusqu'à appeler la »Gazette« un »périodique officiel« bien qu'il admette que la Fronde ait 
français de l'Ancien Régime, un cas particulier9. Sa fondation remonte à Théophraste Renaudot, médecin originaire de Loudun qui s'était installé à Paris en $1612^{10}$ et qui y avait fondé, en 1630 , un »Bureau d'adresse et de rencontre «11; entraîné par son succès, Renaudot entreprit ensuite la publication d'une feuille d'annonces, et, ainsi entré dans le giron de l'édition, se mit à faire paraître, dès 1631, la »Gazette de France «, peut-être le premier périodique français ${ }^{12}$, un hebdomadaire avec de nombreux suppléments »extraordinaires«, qui informait le public des principaux événements politiques en France et surtout à l'étranger. Fondé par un »médecin et conseiller du roi «, à l'instigation de Richelieu, qui rédigea des articles entiers pour ce journal, et pourvu d'un privilège royal exorbitant ${ }^{13}$, cet

marqué »la fin du monopole« de Renaudot, Roger CHARTIER, chapitre »Pamphlets et gazettes«, dans: ID., Henri-Jean MARTIN (dir.), Histoire de l'édition française, t. I: Le livre conquérant. Du Moyen Âge au milieu du XVIIe siècle, [Paris] 1989, p. 501-526, ici p. 516-517.

${ }^{9}$ Au sujet de la »Gazette«, à l'époque considérée, cf. surtout Stéphane HAFFEMEYER, L'information dans la France du XVIIle siècle. La Gazette de Renaudot de 1647 à 1663, Paris 2002 (Bibliothèque d'histoire moderne et contemporaine, 6), notamment p. 591-597 et 600602 sur les articles que la "Gazette« a consacrés au congrès de la paix de Westphalie. Voir aussi Gilles FEYEL, article »Gazette [de France]«, dans: Jean SGARD (dir.), Dictionnaire des journaux. 1600-1789, 2 vol., Paris, Oxford 1991 (Dictionnaire de la presse 1600-1789, 1), t. I, p. 443-449. Dans le contexte de la problématique étudiée, il paraît toujours utile de se reporter, en outre, à Marie-Noële Grand-MESNIL, Mazarin, la Fronde et la presse. 1647-1649, Paris 1967 (Kiosque, 31); Roland Mousnier, Paris capitale au temps de Richelieu et de Mazarin, Paris 1978, en particulier p. 142-143; BRAUN, Die »Gazette de France«.

10 À propos de la biographie de Renaudot, voir notamment Gilles FEYEL, article »Renaudot, Théophraste «, dans: Jean SGARD (dir.), Dictionnaire des journalistes. 1600-1789, 2 vol., Oxford 1999 (Dictionnaire de la presse 1600-1789, 2), t. II, no 677, p. 838-848; Gérard JUBERT (éd.), Père des journalistes et médecin des pauvres. Théophraste Renaudot (1586-1653). Corpus de textes, Paris 2005; parmi les publications plus anciennes, on consultera de préférence: Arsène-Marie CHAUCHAT, La curieuse et grande figure de Théophraste Renaudot sieur de Boissemé, fondateur du journalisme en France, Paris 1937 (Histoire de la presse en dix portraits, 1); Howard M. Solomon, Public Welfare, Science, and Propaganda in Seventeenth Century France. The Innovations of Théophraste Renaudot, Princeton/N. J. 1972; G[érard] JUBERT, La Légende dorée de Théophraste Renaudot, dans: Bulletin de la Société des antiquaires de l'Ouest, 4e série, 16 (1981), p. 141-162; Cahiers de l'Institut français de presse 1 (1987): Théophraste Renaudot. L'homme, le médecin, le journaliste. 1586-1986. Colloque tenu le 29 novembre 1986 sous la présidence de Jean Imbert.

11 Soit un office de publicité ou bien, d'après une expression forgée par Trenard, »une sorte d'agence de petites annonces avec répertoire d'offres et de demandes les plus diverses «, cf. Louis Trenard, La presse française des origines à 1788, dans: Claude Bellanger et al. (dir.), Histoire générale de la presse française, t. I, Paris 1969, p. 27-402, ici p. 84.

12 La question de savoir si la »Gazette« est le premier périodique français, a été l'objet d'une vive controverse: cf. Folke DAHL, Fanny PETIBON, Marguerite Boulet, Les débuts de la presse française. Nouveaux aperçus, Göteborg, Paris 1951 (Acta Bibliothecae Gotoburgensis, 4); et la réfutation des thèses formulées dans ce livre par Gilles FEYEL, article »Nouvelles ordinaires de divers endroits «, dans: SGARD (dir.), Dictionnaire des journaux, t. II, p. 967970, ici p. 969-970. Voir aussi Louis TRENARD, La presse française, p. 80-83.

13 Mousnier, Paris capitale, p. 142; Gilles FeYEL, Richelieu et'la Gazette, dans: Richelieu et le monde de l'esprit, [édité par:] Chancellerie des universités de Paris et Académie française. [Catalogue de l'exposition en] Sorbonne, novembre 1985, Paris 1985, p. 207-216; Gilles FEYEL, Richelieu et la Gazette. Aux origines de la presse de propagande, dans: Roland 
instrument de propagande suggérait, selon Roland Mousnier, par son "choix et une présentation habiles des nouvelles [...] aux lecteurs les conclusions du gouvernement « ${ }^{14}$. Toutefois, Renaudot ne s'avère pas uniquement un exécutant des ordres gouvernementaux; l'emprise du pouvoir sur sa publication n'est que ponctuelle. Il arrive même que l'éditeur contrarie ses desseins ${ }^{15}$. En revanche, il servit à plusieurs reprises à soutenir la politique française durant le congrès de Westphalie.

Il semble bien que ce soit dans la »Gazette de France«, dont les »Extraordinaires « servaient souvent à la publication de documents officiels ${ }^{16}$, que les traités de Westphalie paraissent pour la première fois en langue française. Le numéro du 13 novembre $16488^{17}$, qui en fait connaître la signature, signale que le texte des traités sera prochainement connu à Paris. C'est quatre jours plus tard que la »Gazette« donne un abrégé en français du traité de Münster, divisé en quatre-vingt-quatre articles $^{18}$, qui est suivi, fin novembre et début décembre, par un sommaire du traité d'Osnabrück publié en deux parties ${ }^{19}$. L'antériorité de la publication dans la $\gg \mathrm{Ga}$ zette « par rapport aux autres éditions qui datent de 1648, et qui reproduisent en gros la même traduction, est sinon certaine, du moins probable ${ }^{20}$ : tout d'abord par

Mousnier (dir.), Richelieu et la culture. Actes du colloque international en Sorbonne. [Avant-titre:] 1585-1985. Quatrième centenaire de la naissance de Richelieu, Paris 1987, p. 103-123.

14 MOUSNIER, Paris capitale, p. 143. La correspondance entre le gouvernement et les ambassadeurs français en Westphalie permet d'ailleurs de constater que l'on se servait de la »Gazette « comme moyen de propagande, en y faisant publier des articles quand cela paraissait opportun, par exemple à l'automne 1647, après la levée du siège de Lérida, cf. BRAUN, Die »Gazette de France«, p. 283-284, n. 5.

15 En effet, quand un article de Renaudot était contraire aux intentions du gouvernement, celui-ci le rappelait à l'ordre, cf. le chapitre précédent.

16 Notamment des traités de paix internationaux. Ce n'est donc pas par hasard que la »Gazette«, $\mathrm{n}^{\circ} 128$ de l'année 1649 (29 octobre), p. 973-984, publie la convention entre l'empereur, l'Empire et la Suède, Nuremberg, 28 août 1649 . Cf. aussi TRENARD, La presse française, p. 90-92 sur la présentation de la »Gazette«, et notamment sur ces "Suppléments« et ses »Extraordinaires «; sur ces derniers, il observe: »les Extraordinaires ressemblent aux occasionnels: ils contiennent des documents officiels, des récits détaillés, des relations de sièges « (p. 92).

17 Gazette de France, $\mathrm{n}^{\circ} 171$ de l'année 1648.

18 Gazette de France, $n^{\circ} 174$ du 17 novembre 1648, intitulé: Sommaire des articles de la paix generale entre l'Empire \& la France, p. 1541-1560.

19 Ibid., no 177 du 25 novembre 1648, intitulé: Abregé du traité \& des articles de paix arrestez à Osnabruk entre les plénipotentiaires de l'Empéreur \& de la Reine \& couronne de Süede, en présence des estats de l'Empire, p. 1585-1604; et no 181 du 2 décembre 1648, intitulé: Svite de l'abregé dv traité et des articles de paix, d'entre l'Empire \& la Süéde, p. 1629-1644. ${ }^{20}$ Sur les éditions isolées des traités de paix de l'Ancien Régime conservées à la Bibliothèque nationale de France, cf. Madeleine RÉMY-LIMOUSIN, Traités internationaux de l'Ancien Régime. Éditions isolées et recueils conservés à la Bibliothèque nationale de France. Catalogue, Paris 1997. Il résulte de ce catalogue que dans notre première publication sur les traductions françaises des traités de Westphalie, parue avant la publication de ce catalogue, nous avions déjà repéré toutes les éditions isolées des traités de Westphalie et les recueils des traités de paix concernés, cf. Braun, Traductions (1996). Toutefois, nous avons depuis décou- 
le bref délai entre l'annonce de la signature de la paix et sa publication dans ce périodique; deuxièmement parce que la plupart des autres éditions se fondent sur une »copie imprimée à Paris« et ne peuvent donc pas être les premières à faire connaître le contenu des traités, et qu'elles reprennent la préface ou le commentaire que la »Gazette« avait ajoutés au texte. Il y a toutefois une édition du traité de Münster qui échappe à cette logique puisqu'elle ne donne que le texte seul du traité, sans faire référence à un imprimé antérieur ${ }^{21}$. Mais par le fait même qu'elle publie le traité sans aucune présentation ni introduction, elle suppose qu'il soit déjà assez bien connu dans Paris, et par conséquent elle semble postérieure au numéro de la »Gazette «22.

Quoi qu'il en soit, tous les sommaires parus dans les deux années suivant la conclusion de la paix offrent, abstraction faite de quelques fautes typographiques et changements mineurs, une traduction identique; ceci est vrai pour les sommaires du traité d'Osnabrück aussi bien que pour ceux du traité de Münster. Et il s'agit déjà partiellement, au moins pour les articles les plus importants, d'une véritable traduction plutôt que d'un résumé23. On conserve même les débuts des paragraphes du traité d'Osnabrück cités dans celui de Münster, qui sont laissés en latin. Ainsi, une édition du sommaire du traité de Münster comporte une vingtaine de pages in-4o; celui du traité d'Osnabrück occupe environ trente-cinq pages d'une édition du même format bien que les articles contenus de même dans le

vert trois autres éditions, qui ne figurent pas dans ce catalogue, n'appartenant pas à la Bibliothèque nationale de France ou figurant dans des ouvrages sur le droit d'Allemagne qui n'y sont pas pris en compte.

${ }^{21}$ Il s'agit du »Sommaire des articles de la paix generale entre l'Empire et la France«, publié à Paris en 1648 par les »Imprimeurs et Libraires ordinaires du Roy«; Sommaire des articles de la paix generale entre l'Empire et la France, Paris 1648, cf. RémY-Limousin, Traités internationaux, no 196. "Sommaire« réimprimé à Paris, en 1649, cf. RÉMY-Limousin, Traités internationaux, $\mathrm{n}^{\circ} 197$.

22 Il est à noter qu'à Paris, on connaissait aussi les éditions isolées publiées à l'étranger et/ou en langue étrangère en 1648 et 1649; en effet, la Bibliothèque nationale conserve, outre les éditions françaises, des éditions latines, néerlandaises et allemandes; cf. RÉMY-LıMOusıN, Traités internationaux, $\mathrm{n}^{\mathrm{os}} 184,185,186,187,188,189,200,202,203,204,206$. On continuait d'imprimer la paix de Westphalie en latin à Paris (pour le traité de Münster, Sébastien Cramoisy 1656), ibid., $\mathrm{n}^{\circ}$ 201. Cependant, un recueil de lois et de traités (avec un frontispice s.l.n.d.) que Rémy-Limousin dit avoir été publié en France après 1658 est en fait la fin du deuxième tome de l'édition de HEISs, Histoire de l'Empire (1684), partie paginée en chiffres romains (voir ci-dessous); RÉmY-Limousin, Traités internationaux, $n^{\circ} 191$; ibid., $n^{\text {os }} 192$, 193 et 194: les derniers tomes de HeIss, Histoire de l'Empire (éditions de 1685 et de 1694, voir ci-dessous) sont présentés comme des publications indépendantes. Par ailleurs, on notera l'absence d'éditions isolées du traité final passé par l'empereur avec la Suède le 24 octobre 1648.

${ }^{23}$ De ce point de vue, il convient de nuancer le jugement de Vast sur le "Sommaire des articles«; en fait, cet auteur n'y voit "qu'un arrangement abrégé sur certains points et non une traduction littérale du traité «, Henri VAST (éd.), Les grands traités du règne de Louis XIV, [t. I:] Traité de Munster, Ligue du Rhin, Traité des Pyrénées (1648-1659), Paris 1893 (Collection de textes pour servir à l'étude et à l'enseignement de l'histoire), p. 11, n. 1. En effet, tout dépend de ce qu'on attend d'un tel texte: certes, il n'offre pas (et ne veut même pas offrir, selon son titre) une traduction complète, pourtant aucun point essentiel ne lui échappe. 
traité de Münster n'y figurent pas: pour eux, on renvoie à l'édition de ce dernier traité. L'édition de la paix d'Osnabrück suit la répartition originale du texte latin en dix-sept articles ${ }^{24}$, tandis que le traité de Münster, initialement sans numérotation d'articles ${ }^{25}$, est divisé en quatre-vingt-quatre paragraphes ${ }^{26}$. La $\gg$ Gazette« accompagne ce dernier d'une préface ${ }^{27}$ et d'un bref commentaire à la fin du texte du traité de paix ${ }^{28}$. La paix d'Osnabrück est publiée sans préface, mais avec quelques remarques qui servent de transition entre la première partie du traité (du préambule à l'article $\mathrm{V}$, publiée dans la »Gazette« du 25 novembre) et la deuxième partie (articles VI à XVII, publiée dans la »Gazette« du 2 décembre); de plus, on y

${ }^{24}$ Comme l'article XVII du traité d'Osnabrück correspond pour la plus grande partie littéralement aux derniers paragraphes du traité de Münster, l'imprimé de ce premier finit par l'article XVI.

${ }^{25}$ Pour le traité de Münster, on peut parler de »paragraphes « aussi bien que d'»articles«. Par contre, pour le traité d'Osnabriuck, il faut bien faire la distinction entre ces deux termes puisqu'il est divisé en articles, et subdivisé en paragraphes ou alinéas.

26 Il est important de noter cette disposition, car cette subdivision, inconnue en Allemagne, s'imposera dans une grande partie des éditions françaises publiées avant 1789.

27 Cette préface démontre que l'écrit fut bien considéré à l'époque comme une arme efficace dans le combat politique; cf. Gazette ${ }^{\text {os }} 174,1648$, p. 1541-1542.

28 À cet endroit, nous retrouvons encore les mêmes préoccupations et le même soulagement (au sujet de l'Espagne) que nous avons déjà fait ressortir d'un autre numéro de la »Gazette«, ibid., p. 1560. Pourtant, l'affaire du secours éventuel de l'empereur pour le Roi Catholique n'était pas aussi claire que le prétend la »Gazette «, et les Français en étaient conscients. Dans la correspondance politique se trouve une »Censure envoyée de Paris sur le traité de la paix de l'Empire, avec le mémoire de monsieur Servien du $27^{\mathrm{e}}$ octobre 1648 « où l'on critique vivement la teneur de cet article: »En second lieu, l'article du secours de l'Empereur est conceu de telle façon qu'il ne tiendra qu'aux Espagnols que demain ils ne donnent juste préteste [sic] aux Allemans de les assister: car il est dit seulement que l'Empereur et les princes de l'Empire ne se mesleront point des différends qui sont à présent entre les couronnes de France et d'Espagne; mais que pour les différens qui surviendront à l'advenir, chacun pourra assister le parti qu'il voudra, de sorte que le Roy d'Espagne n'aura qu'à déclarer de nouveau la guerre au Roy [...], et voilà aussy tost l'Empereur en droict par le traicté de secourir l'Espagnol « (meilleure copie de cette »Censure «: AE, CP All. 110'fol. 533-534', ici fol. 533'534 , le titre d'après une note fol. 534', qui est de la main de Lionne; autre copie, intitulée "Escrit contre le traité de l'Empire « et datée »octobre 1648«: AE, CP All. 122 fol. 585-585', note fol. 585' de la main d'Alard [secrétaire de Servien]: »Escript envoyé de Paris à monsieur Godefroy, contre le traicté de l'Empire «). Selon Vast, cette lettre non signée serait du comte d'Avaux, cf. VAST, Les grands traités, p. 7. Servien l'attribuait, sans donner le nom complet de l'auteur, à un certain »M.r D.« (AE, CP All. 122 fol. 590), ce qui permet bien de lire » $\mathrm{M}^{\mathrm{r}} \mathrm{D}[$ 'Avaux]"; en effet, c'est par cette abréviation que Servien désignait généralement son (ancien) collègue. Cf. la lettre de Servien à Lionne, 25 octobre 1648, copie: AE, CP All. 122 fol. 590-591, en particulier fol. 590-590': "L'on a déjà envoyé depuis le bonhomme Godefroy une censure contre le traitté de l'Empire qui va faire voir ce que je vous mandois l'autre jour que ceux qui crioient le plus de ce que la paix ne se faisoit point seront les premiers à en blasmer les conditions quand elle sera faitte. Il y a un article qui vous fera voir qu'elle vient de chez M. ${ }^{r}$ D. n'y ayant personne que luy en France qui aimast mieux donner aux ministres de Suède qu'à ceux du Roy la gloire de ce qui a esté obtenu dans ce traitté à notre avantage «. TISCHER confirme cette attribution: il s'agirait d'une lettre adressée par d'Avaux à Godefroy; EAD., Französische Diplomatie, p. 114-115, en particulier p. 114, n. 77 . 
a ajouté les deux articles suspensifs présentés le 6 août 1648 par les plénipotentiaires suédois, qui avaient déclaré que le traité d'Osnabrück n'entrerait en vigueur que le jour de la conclusion du traité entre l'empereur et la France, et sous condition que la Hesse-Cassel fût déchargée du dédommagement de la milice suédoise $^{29}$. Il est important de comprendre quelle technique de traduction est à la base de ces »sommaires« ou »abrégés « 30 ; car, au lieu de résumer tous les articles, le traducteur - dont l'identité précise nous échappe - traduit littéralement les clauses qu'il considère comme les plus importantes, en ne mentionnant pas du tout celles qui sont jugées secondaires. Ce procédé se manifeste tout particulièrement dans son résumé du traité de Münster. En revanche, on peut dire que, quelques exceptions négligeables mises à part, aucun article entier ne manque dans ces éditions, et c'est notamment la paix d'Osnabrück qui, pour les articles non contenus dans celle de Münster, a été traduite d'une façon assez complète. Quant au traité de Münster, ce sont avant tout les paragraphes concernant les aspects techniques de son exécution qui sont résumés assez brièvement; de même, on n'évoque pas les dispositions particulières qui concernent l'exécution des articles réglant le dédommagement de la maison de Hesse-Cassel qui, eux-aussi, ont un caractère assez technique, et qui étaient probablement peu intéressants pour un lecteur français $^{31}$. La division en paragraphes semble parfois fort discutable. Cependant, si l'on se pose la question de savoir si le traducteur a bien compris ce dont il parlait, on peut bien y répondre de manière positive, même si cette traduction n'est pas parfaite ${ }^{32}$. Cependant, sur les points essentiels, le résumé du traité de Münster offrait aux lecteurs contemporains des idées assez justes bien que le traducteur n'ait probablement eu que fort peu de temps pour accomplir sa tâche ${ }^{33}$.

${ }^{29}$ Ces textes sont publiés en latin par MeIERN, Acta Pacis Westphalicae Publica, t. VI, p. 124 et 173. À propos des négociations épineuses sur le problème de la satisfactio militiae qui traînaient jusqu'à la veille de la signature du traité d'Osnabrück, cf. le résumé de OsCHMANN, Der Nürnberger Exekutionstag, p. 85-94.

${ }^{30} \mathrm{Ce}$ sont les deux termes employés à l'époque. Le mot »résumé«, rappelons-le, n'apparaîtra en français qu'en 1750 .

31 Il s'agit, selon la numérotation actuelle, des $\S \S 53-57$ de l'IPM = art. XV,6-12 IPO, texte latin: OSCHMANN, APW III B 1/1, p. 16-18, 148-149.

32 Par exemple, certaines fautes s'y sont glissées à cause de la technique d'»abréviation « que nous avons évoquée. En outre, en ce qui concerne le fameux $\$ 87$ du traité de Münster, on peut se demander si le traducteur a bien mesuré la portée de cette clause, parce que sa traduction est on ne peut plus défavorable au roi de France. Il en traduit littéralement la première partie assurant aux ordres en Alsace qu'ils conserveront leur statut d'états immédiats de l'Empire, mais n'évoque point sa deuxième partie qui réserve au roi de France sa souveraineté sur ses possessions en Alsace. Cf. $\$ 87$ IPM, selon la numérotation chez Antje Oschmann, APW III B 1/1, p. 26. Sur cet article cf. Dickmann, Der Westfälische Frieden, passim (voir l'index, p. 614).

33 Certains changements de `dernière minute $<$ intervenus quand la "Gazette « était déjà sous presse attestent cette pression; nous sommes arrivés à cette conclusion en comparant les exemplaires suivants: BNF $4^{\circ} \mathrm{Lc} c^{2} .1$; bibliothèque de l'Arsenal $4^{\circ} \mathrm{H} .8917$; bibliothèque de ' l'Institut $4^{\circ} \mathrm{S} 260$; bibliothèque Mazarine 16301*; bibliothèque du Sénat $J 221$; de plus, l'édition lyonnaise de la deuxième partie du traité d'Osnabrück (AE, CP All. 110 fol. 489506': IPO, l'exemplaire de la Gazette $n^{\circ} 181$, qui provient de l'édition lyonnaise du 10 dé- 
Si la traduction de ce traité est quand même, somme toute, satisfaisante, le résultat de celle du traité d'Osnabrück est assez mitigé. Les articles réglant les questions territoriales (Suède et princes allemands) sont très bien traduits, et parfois même tous les traducteurs postérieurs n'ont pas su faire mieux. Par contre, la traduction de l'article V, le cœur même du traité d'Osnabrück, relatif au droit de religion, est tellement truffée d'erreurs qu'il est souvent très difficile de retrouver les paragraphes de l'original que le traducteur aurait voulu traduire. De toute évidence, il ne sait point ce que veulent dire des notions aussi fondamentales du droit de religion allemand que le ius reformandi ou l'itio in partes ${ }^{34}$. En outre, un grand nombre de paragraphes entiers, qui sont parmi les plus importants de tout l'article, manquent dans cette traduction, notamment presque tous ceux qui se réfèrent au ius reformandi ${ }^{35}$. Or, d'après nos recherches, ce conglomérat de lacunes, de nonsens et de contre-sens est la seule »traduction « de l'article de religion dont disposeront les Français pendant plus de trente-cinq ans, jusqu'à la parution de l'»Histoire de l'Empire « de Jean Heiss, en 1684.

En 1648, l'édition des traités de Westphalie représenta d'ailleurs un intérêt économique majeur, de sorte que même la Curie romaine se vit renseignée sur les disputes qui existaient alors entre Sébastien Cramoisy et Théophraste Renaudot à ce sujet. En effet, le nonce du pape à Münster, le médiateur Fabio Chigi, transmit le 27 novembre 1648 les nouvelles qu'il avait reçues de Paris le 13 et qui disaient, entre autres: »Tandis que Cramoisy imprimeur du Roi et Renaudot, le gazettier, se disputent l'impression des articles de la paix signée et publiée entre l'Empire et les couronnes alliées, monsieur l'ambassadeur de Suède en a fait imprimer de nombreux exemplaires et les distribue à ses amis « ${ }^{36}$. L'intérêt porté à ces traités est donc incontestable ${ }^{37}$.

cembre 1648, imprimée chez Jean-Aymé Candy, imprimeur ordinaire du roi); sur la »Gazette « à Lyon, cf. Gilles FEYEL, La "Gazette« en province à travers ses réimpressions, 16311752. Une recherche analytique de la diffusion d'un ancien périodique dans toute la France. Avec un aperçu général et bibliographique pour chacun des centres de réimpression de la $\mathrm{Ga}$ zette, Amsterdam, Maarssen 1982 (Études de l'Institut de recherches des relations intellectuelles entre les pays de l'Europe occidentale au XVIIe siècle, Nimègue [SIB], 8), ici p. 292313. Selon les résultats que nous avons ainsi obtenus, le résumé du traité de Münster et la deuxième partie de l'abrégé du traité d'Osnabrück n'ont pas connu de modifications majeures, tandis que les différents exemplaires de la première partie du traité d'Osnabrück font état de leçons divergentes parfois importantes. Ces modifications sont documentées dans la réédition des résumés de la »Gazette «, cf. Braun, OsChMANN, REPGEN, APW III B 1/2.

34 Pour ces concepts et leur signification dans les traités de Westphalie, cf. Croxton, TrSCHER, The Peace of Westphalia, p. 140 et 142-143 (avec des références bibliographiques). Sur le ius reformandi, cf, toujours Burkhard von BonIN, Die praktische Bedeutung des ius reformandi. Eine rechtsgeschichtliche Studie, Stuttgart 1902 (réimprimé à Amsterdam 1962; Kirchenrechtliche Abhandlungen, 1); sur l'itio in partes: Martin HECKEL, Itio in partes: Zur Religionsverfassung des Heiligen Römischen Reiches Deutscher Nation, dans: Zeitschrift der Savigny-Stiftung für Rechtsgeschichte, Kanonistische Abteilung 64 (1978), p. 180-308. ${ }^{35}$ Le traducteur ne semble comprendre »ius reformandi« que lorsqu'il est précisé par »exercitium religionis « tandis que l'ellipse en fait une chose incompréhensible pour lui.

36 Cf. Archivio Segreto Vaticano, Segreteria di Stato, Nunziatura per le Paci, t. 23 fol. 732 733': avis »Di Parigi, li 13 di novembre 1648 « (envoyé à Rome avec la dépêche de Chigi au 
Or, combien d'argent un lecteur devait-il payer pour acheter les numéros de la "Gazette « où les traités de Westphalie sont publiés? Les trois numéros comprennent au total cinquante-six pages. Si l'on concède que les prix, que Gilles Feyel a indiqués pour la vente des "Extraordinaires « de la "Gazette en $1650^{38}$, sont à peu près les mêmes qu'en 1648, l'on peut supposer que le traité de Münster a été vendu aux colporteurs au prix de cinq sous, les deux parties du traité d'Osnabrück respectivement pour cinq et quatre sous. En ajoutant la marge des colporteurs dont le profit s'élevait, en général, environ à un quart du prix de la vente aux particuliers, le prix total des trois numéros de la »Gazette« était de dix-huit sous et demi à dix-neuf sous, c'est-à-dire qu'un Parisien devait payer presque une livre

secrétaire d'État Panzirolo, de Münster, le 27 novembre 1648, original: ibid., fol. 730), ici fol. 733-733': »Mentre litigano insiem il Cramoisy stampatore regio et il Renaudot gazzettante a chi di loro s'aspetti l'impressione de' capitoli della pace firmata, e pubblicata tra l'Imperio, e le corone collegate; il signor ambasciatore di Svezia ne ha fatti stampare molti esemplari, e li va distribuendo a' suoi amici«. Le soin avec lequel Servien veilla à l'impression du traité est également mentionné par le nonce: ibid., fol. 695-697', rapport du nonce, de Münster, le 6 novembre 1648, ici fol. 695: »J'envoie aussi l'imprimé du traité de paix que les deux parties ont signé et qu'elles ont fait, ensuite, imprimer à Münster; mais monsieur Servien, avec sa précision habituelle, fit changer et imprimer de nouveau le frontispice, ajoutant le titre de Majesté et de Sacré pour son Roi, afin de le mettre de pair avec celui de l'Empereur, puisque, auparavant, on l'avait seulement traité de Roi de France (»Mando anco la stampa dell'istromento di pace, che concordemente ambe due queste parti soscrissero, e che poi hanno fatto stampare in Munster; di cui però il signor Servien con la sua accuratezza fece mutare, e ristampare il frontispizio, con aggiognervi il titolo di Maestà, e di Sacra per il suo re, e col metterlo al pari con quello dell'Imperatore, che per avanti era stato messo con la sola enunciatione di Regis Galliarum«). Dans ce contexte, il est à noter que les éditions imprimées en France utilisent l'expression »la France«, sans référence au roi.

37 En 1660, le privilège de la publication des documents officiels ne fut accordé qu'à cinq imprimeurs du roi, dont Sébastien Cramoisy; cf. la brochure contenant le texte de la paix des Pyrénées: Traitte de paix entre les couronnes de France et d'Espagne: avec le contract de mariage du roy tres-chrestien et de la serenissime infante fille aisnée du Roy Catholique. Le 7. novembre 1659. Avec l'explication de l'article XLII. du susdit traitté, concernant le Roussillon: Du 31. may 1660 . Leus, publiez \& registrez en parlement, chambre des comptes, \& cour des aides, és mois de iuillet \& d'aoust en suivans, Paris, Imprimeurs \& Libraires du

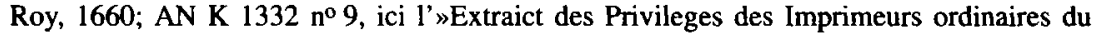
Roy«, p. [88]: »Par Arrest de la Cour du 24. Octobre 1648. donné en consequence de la Declaration du Roy verifiée en Parlement, Chambre des Comptes, Cour des Aydes, Chastelet \& Baillage du Palais, \& autres Arrests confirmatifs; il n'est permis qu'à Antoine Estienne, Sebastien Cramoisy, Pierre Roccolet, Pierre le Petit \& Iacques Langlois, seuls Imprimeurs ordinaires de Sa Majesté, d'imprimer toutes les affaires du Roy portées par ladite Declaration: Et défenses sont faites à tous autres Imprimeurs, mesme à ceux se disans pourveus par Brevets, de les imprimer ou contrefaire, sur peine de faux $\&$ de 500 . livres d'amende: Et en cas de contravention, la peine desdites 500 . livres portée par icelle Deciaration, dés à present encourue; Et cependant permis de saisir, sceller ou transporter les Impressions, Presses \& Caracteres des contrevenans, nonobstant lesdits Brevets \& autres oppositions quelconques: Et encore tant par ledit Arrest que autres, sont faites les défenses à tous Colporteurs \& autres, d'en vendre \& debiter, ny s'en trouver saisis, sur les mesmes peines, \& emprisonnement de leurs personnes «.

38 FEYEL, Gazette, p. 445. 
pour acheter les traités de Westphalie ${ }^{39}$. Il est donc évident que dans la capitale il y avait un marché suffisant pour débiter les exemplaires imprimés et une demande de les acheter. Cela nous mène à aborder le problème du tirage. En 1638, Renaudot indiquait lui-même que la "Gazette " était généralement tirée à mille deux cents exemplaires. En ajoutant les réimpressions en province que Renaudot n'a pas prises en compte dans son calcul en 1638, Feyel pense que le tirage de la »Gazette«, en 1670, s'est situé entre trois mille quatre cents et quatre mille huit cents exemplaires. Mais l'intérêt que les lecteurs ont porté à la »Gazette de France«, en 1648, se manifeste non seulement dans le tirage, mais aussi dans la multiplication évidente du nombre de numéros et de pages. En effet, en 1638 et en 1670, la »Gazette « est composée respectivement de sept cent soixante-douze pages et de mille deux cent quarante pages. L'année 1648 comprend mille sept cent soixante-dixhuit pages. Dans l'histoire de la "Gazette«, il n'y avait jamais eu d'année aussi riche, et, jusqu'à la Révolution, la »Gazette« n'atteindra plus jamais ce nombre de pages. L'on peut supposer que ce phénomène s'explique par deux facteurs: premièrement par la Fronde; deuxièmement par la guerre menée contre l'Espagne et contre l'empereur, puis par la conclusion de la paix avec ce dernier. Feyel remarque à juste titre: »les guerres de l'ancien régime ont multiplié les lecteurs de la Gazette, cependant que la paix paraît les avoir démobilisés $« 40$. Cela semble vrai quand la paix dure longtemps, mais quand on vient de la conclure, le public s'intéresse encore aux nouvelles du théâtre des négociations.

Nous devons encore répondre à une question essentielle: qui aurait traduit les traités de Westphalie pour la »Gazette«? Sans que nous puissions déterminer avec certitude l'identité du traducteur, il semble possible de formuler des hypothèses. À supposer que le résumé du traité de Münster et les deux parties de l'abrégé de la paix d'Osnabrück aient été rédigés par la même personne, nous pensons qu'il y a trois hypothèses qui paraissent vraisemblables: selon la première hypothèse, la traduction aurait été faite au sein de la rédaction de la »Gazette«. Selon la deuxième, elle proviendrait des chancelleries de la délégation française à Münster. Or, si nous savons bien que des articles ont été publiés dans la »Gazette «à l'instigation de l'ambassade française de Münster et du gouvernement, et que la chancellerie de Servien préparait, par exemple, des traductions de certains documents pour les faire publier sous forme de brochures ${ }^{41}$, nous n'avons, jusqu'à présent, aucun exemple servant à démontrer qu'on fournit des traductions à Renaudot. De plus, du point de vue terminologique, la correspondance entre la délégation française en Westphalie et le gouvernement nous semble parfois différer sensiblement de la manière de traduire de ce traducteur anonyme, s'écartant moins de la terminologie latine en usage dans l'Empire. Étant donné que le travail de traduction des collaborateurs de Renaudot est bien attesté, la première hypothèse semble être la plus plausible. Si on l'admettait donc, on pourrait imaginer comme traducteur un

${ }^{39} \mathrm{La}$ livre tournois vaut 20 sous.

40 FEYEL, Gazette, p. 446.

${ }^{41}$ Cf., par exemple, Braun, APW II B 5/2, p. 1191-1192. 
rédacteur, responsable de la traduction des journaux en provenance de l'Allemagne, voire Renaudot en personne. Toutefois, la solution la plus simple consiste à attribuer la traduction à un collaborateur, puisque l'on sait que des collaborateurs faisaient régulièrement des traductions pour Renaudot. C'est donc l'hypothèse la plus plausible, mais rien ne permet de la prouver, en l'état actuel de nos connaissances. En revanche, nous pouvons exclure une troisième hypothèse selon laquelle ce collaborateur de Renaudot serait Jean Epstein ${ }^{42}$. Ce dernier avait la charge de traduire les journaux allemands pour Renaudot: selon Gilles Feyel, ce personnage possédait à Paris un »véritable bureau de traduction des gazettes étrangères, venues des Pays-Bas et d'Allemagne « 43 . Epstein, bourgeois de Paris et naturalisé Français en 1639 ou en 1640, était d'origine allemande et de religion calviniste. On sait qu'il proposait, dans la deuxième moitié des années 1630 , une gazette manuscrite, reçue par exemple par le duc Bernard de Saxe-Weimar en 1636. Il transmettait à la »nation« allemande de l'université d'Orléans les nouvelles qu'il recevait d'Allemagne ${ }^{44}$. À côté de ses entreprises personnelles, Epstein s'était associé depuis 1631 à des imprimeurs et libraires parisiens. En effet, le 9 juillet 1631, il prend un engagement par acte notarié qui l'oblige à fournir ses traductions à l'imprimeur Jean Martin et au libraire Louis Vendosme, qui publiaient les »Nouvelles ordinaires«, concurrentes de la »Gazette « de Renaudot ${ }^{45}$. Cet acte précise d'une manière très détaillée l'engagement pris par Epstein:

Ledict sieur Epstein a promis et promect faire venir et apporter toutes les septmaines d'Allemagne les nouvelles courantes communément appellées Gazettes, les rendre traduictes et translatées en françois en telle sorte qu'elles puissent estre receues et avoir cours - à commencer vendredy unziesme jour des presens moys et an et continuer de la en avant par chacune sepmaine audict jour. Ce qu'il effectuera tous les jeudys avant midy pour estre si faire se peult le mesme jour imprimées et assistera icelluy sieur Epstein à l'impression et correction desdictes nouvelles pour estre lesdictes nouvelles vendues et distribuées le vendredy de bonne heure.

Cet engagement ne dura pas longtemps; en fait, dès 1631, Renaudot parvient à s'accorder à Epstein et l'engage à »luy fournir les Memoires et Traductions qu'il fournissoit ausdits Imprimeurs« Martin et Vendosme, si l'on en croit la requête que ces derniers présentèrent au mois d'octobre 1634 , se plaignant de Renaudot ${ }^{46}$. Selon Feyel, il est certain qu'Epstein continuait ses traductions au profit de Re-

42 Nous l'avons formulée il y a quelques années, tout en concluant que la question de savoir qui était le traducteur des traités restait ouverte; cf. BRAUN, Die »Gazette de France«, p. 287. ${ }^{43}$ FEYEL, Nouvelles ordinaires, p. 967 (citation) et p. 968 . Sur Epstein, voir surtout ID., article $»$ Epstein, Jean «, dans: SGARD (dir.), Dictionnaire des joumalistes, t. I, no 291, p. 378379. Sur Epstein en tant que traducteur au service de Renaudot, cf. aussi TRENARD, La presse française, p. 81 (qui l'appelle »Epsin«); CHARTIER, Pamphlets et gazettes, p. 511 et 516 (il le nomme Louis Epstein ou Epstin); FEYEL, Gazette [de France], p. 447.

44 Cf. J[ules Michel Henri] Mathorez, Les étrangers en France sous l'Ancien Régime. Histoire de la formation de la population française, vol. II, Paris 1921, p. 25.

45 Cf. FeYel, Nouvelles ordinaires.

46 Cité ibid. 
naudot et d'autres pourvoyeurs de nouvelles ${ }^{47}$. Chargé de la traduction des gazettes étrangères, ce personnage était apparemment d'autant plus prédestiné à traduire les traités de Westphalie, pour des raisons jusqu'à présent inconnues des spécialistes de l'histoire de la presse, puisqu'il travaillait depuis 1630 environ comme correspondant du gouvernement suédois à Paris ${ }^{48}$ et qu'il entretenait une correspondance avec les ambassadeurs suédois à Osnabrück, au début du congrès de Westphalie ${ }^{49}$. Or, Feyel a tort de prétendre qu'»on ignore tout de ses dates de naissance et de décès « ${ }^{50}$. En effet, nous pouvons prouver que ce collaborateur de Renaudot était déjà mort avant 1648. Cela est attesté à la fois par la correspondance des plénipotentiaires suédois au congrès de Westphalie et par la correspondance de Grotius. D'après cette dernière, Epstein est mort avant le 8 octobre 164451; Rosenhane, résident suédois à Münster, puis ambassadeur à Paris ${ }^{52}$, fut chargé de régler quelques affaires concernant l'héritage, suite au décès d'Epstein $^{53}$. Une contradiction reste cependant à expliquer: d'après Grotius, Epstein était mort au plus tard en 1644, mais Feyel a tiré des Archives nationales un arrêt du Conseil d'État datant du 31 juillet 1646 impliquant sa personne ${ }^{54}$. En revanche, il nous paraît certain qu'Epstein était bien mort en 1648; en effet, abstraction

47 Cf. ID., Epstein.

48 Cf. PALmSTIERNA et al., Histoire de l'administration, p. 99.

49 Cf. WeRMTER, APW II C 1, passim (voir l'index, p. 931).

50 FeYel, Epstein, p. 378.

51 Cf. [Hugo Grotius], Briefwisseling van Hugo Grotius, publié sous la direction de P. C. Molhuysen, puis de B. L. MEULENBRoeK et al., 's-Gravenhage depuis 1928 (depuis le t. III: Rijks Geschiedkundige Publicatiën. Grote serie), t. XVI: okt. 1644 - aug. 1645 (2000), p. 39. 52 Schering Rosenhane (1609-1663), déjà évoqué au chapitre précédent, fut résident suédois à Münster de 1643 à juillet 1647 (date de la lettre de révocation), puis, de 1648 à 1649 , le premier ambassadeur suédois à Paris qui était d'origine suédoise. Â son sujet, $\mathrm{cf}$. aussi PALMSTIERNA et al., Histoire de l'administration, p. 113-114.

53 Cf. KOHL, APW II C 4/1, no 121, p. 121-126: instructions données à l'ambassadeur de Suède en France, Rosenhane, datées du 29 novembre / 9 décembre 1647 à Stockholm, ici p. 126 paragraphe 16. D'après ces instructions, Epstein était mort depuis quelque temps, après avoir rendu, sa vie durant, de bons services à la reine et à la couronne de Suède: „Epstein, som för någon tijdh sedan är blefven dödh och hade i sin lijfztijdh ett vist och åhrliget deputat af Konglige Maijestät och chronan Sverige«. Au moment de prendre en considération l'hypothèse voulant qu'Epstein soit le traducteur des traités de Westphalie, le dernier volume de la correspondance de Grotius (commençant en octobre 1644) n'était pas encore sorti, et nous n'avions pas encore découvert ce paragraphe des instructions données à Rosenhane, puisque les éditeurs n'ont pas identifié le personnage d'Epstein dans la note de bas de page, et ont oublié son nom dans l'index.

54 Acte du Conseil d'État (AN, V4 1499 fol. 160-160') cité par FEYEL, Epstein, p. 379: selon Feyel, il était dirigé contre Epstein »et plusieurs autres " (citation tirée de l'arrêt). Il portait interdiction à ces personnes de faire »ordinairement des assemblées où ils rapportent comme dans un bureau tout ce qu'ils apprennent « et de composer ou copier à la main des »nouvelles à leur fantaisie « débitées en très grande quantité dans tout le royaume (citations tirées de l'arrêt, ibid.). En 2005, cet arrêt a été publié par JUBERT, Père des journalistes, document $n^{\circ} 342$, p. 482-483. On peut exclure une erreur de datation; selon la teneur de l'arrêt, »Epstein« était toujours vivant. S'il est probable que plusieurs personnes portant ce nom ont vécu à ce moment-là à Paris (il n'y a qu'à penser aux héritiers d'Epstein), il n'y a aucune trace d'une deuxième personne ayant exercé les activités de traducteur, de journaliste et de correspondant. 
faite de l'arrêt de 1646, les travaux des spécialistes de l'histoire de la presse n'avancent aucune date postérieure à 1640 concernant ses activités ou sa personne, et la correspondance suédoise confirme sa mort avant le mois de décembre 1647 (sans datation précise du décès). Tous les témoignages concordent: au plus tard en 1647 l'on n'a plus aucune nouvelle de la part d'Epstein puisqu'il est mort. Comme la »Gazette« de Renaudot continua à publier des numéros »extraordinaires « regroupant les nouvelles de l'Europe septentrionale et centrale jusqu'en 1682, il y a fort à parier qu'Epstein ait été remplacé au sein de l'équipe de Renaudot, puisque ce dernier avait besoin d'un tel collaborateur dépouillant les nouvelles (imprimées ou manuscrites) arrivant des pays du Nord et de l'Est. Même si Epstein n'est donc pas le traducteur des traités de Westphalie, les informations que nous avons pu obtenir sur ses conditions de vie et de travail, et notamment l'acte notarié, nous permettent de mieux comprendre dans quelles circonstances le traducteur de l'année 1648 a dû travailler: les versions terminées le matin passaient sous presse vers midi, étaient probablement corrigées par le traducteur et par Renaudot, et devaient être vendues le lendemain matin de bonne heure. Cela explique que nous ayons repéré des changements de sdernière minute en comparant différents exemplaires du même numéro de l'édition parisienne de la »Gazette« de 1648 qui, contrairement à notre attente, ne sont pas tout à fait identiques ${ }^{55}$. Malgré les défauts de ces résumés, il convient toutefois de se rappeler leur objectif: il ne s'agissait que de donner au public une première information sur ce qui avait été conclu entre les princes européens, et non de fournir une édition sscientifique ${ }^{56}$ qui satisfasse aux besoins des juristes. Pour les diplomates et les juristes, ces abrégés ne constituaient pas un instrument de travail fiable; les éditions plus précises, en latin et en français, destinées à leur usage, devaient suivre.

Nous avons déjà évoqué un certain nombre d'éditions isolées qui reprennent la traduction publiée dans la »Gazette «57. Au moins six d'entre elles, parues de 1648 à 1649 , sont parvenues jusqu'à nos jours: cinq du traité de Münster ${ }^{58}$, une seule du

55 À l'édition parisienne s'ajoutent au total trente-huit éditions parues en province, de 1631 à 1752 , cf. FEYEL, La »Gazette« en province.

$56 \AA$ À vrai dire, la première édition prenant en compte tous les originaux et fidèle en tout point n'a été publiée qu'en 1998, cf. OSCHMANN, APW III B 1/1.

57 Sommaire des articles de la paix generale entre l'Empire et la France, Paris, Imprimeurs et Libraires ordinaires du Roy, 1648; réimprimé ibid., en 1649. Sommaire des articles de la paix generale, entre l'Empire et la France. Jouxte la copie imprimée à Paris, Metz, Pierre Colignon, Imprimeur juré du Roy et de ladite Ville [1648 ou 1649].

58 Outre celles qui ont déjà été signalées: Articles de la paix générale d'Allemagne accordez entre l'Empereur et les Estats de l'Empire, le Roy de France, le Royaume de Suède et leurs alliez. Iouxte la copie imprimée, Paris, Bureau d'Adresse 1648; cf. RémY-Limousin, Traités internationaux, $\mathrm{n}^{\circ}$ 195. Le lieu de publication incite à croire que Renaudot fut responsable de cette édition. Sommaire du traité et des articles de la paix générale entre l'Empereur et le Roy tres-chrestien, et leurs alliés. Iouxte la copie imprimée à Paris, [s.l.] 1648 (selon le catalogue de la BNF, peut-être imprimé en France et avec le même matériel typographique que le »Traité de la paix arresté a Osnabruk« [voir la note suivante]; cf. RÉMY-Limousin, Traités internationaux, $\mathrm{n}^{\circ} 198$ ). 
traité d'Osnabrück ${ }^{59}$. Bien que le respect du dépôt légal ait fait des progrès au cours du XVII ${ }^{\text {siècle }}{ }^{60}$ (de sorte que la plupart des publications devraient en principe être conservées aujourd'hui à la Bibliothèque nationale, héritière de l'ancienne Bibliothèque royale), on peut supposer que le nombre total des éditions publiées à cette époque ait été supérieur à ce chiffre. De toute manière, le nombre d'entre elles dont nous disposons encore aujourd'hui est toujours impressionnant. Une constatation s'impose d'emblée: le traité de Münster l'emporte largement sur celui d'Osnabrück. Rappelons que s'il y a au moins cinq éditions isolées du premier, nous n'avons pu en retrouver qu'une seule de la paix d'Osnabrück ${ }^{61}$.

Nous avons dit que, par certains aspects, quelques-unes de ces éditions font penser à des publications quasi officielles. Premièrement, par leur aspect physique: deux d'entre elles portent, sur leur frontispice, les armes de France et de Navarre accolées, avec le $» \mathrm{~L}$ « simple, et timbrées de la couronne royale. Il s'agit du »Sommaire des articles de la paix generale entre l'Empire et la France «, qui date de $1648^{62}$, et des »Articles de la paix générale d'Allemagne accordez entre l'Empereur et les Estats de l'Empire, le Roy de France, le Royaume de Suède et leurs alliez«, parus de même en $1648^{63}$. Toutes les deux sont publiées avec privilège royal. Deuxièmement, les »Articles de la paix générale« ont été imprimés au »Bureau d'adresse« à Paris, qui se trouvait soumis au contrôle du gouvernement; le „Sommaire des articles« l'a été par les »imprimeurs et libraires ordinaires du roi«. L'édition publiée à Metz avec le même titre "Sommaire des articles de la paix générale «64 a été établie par Pierre Colignon, qui exerçait la fonction d'»imprimeur

59 Traité de la paix arresté a Osnabruk. Entre les plénipotentiaires de l'Empereur, et de la Reine et couronne de Suède, en presence des estats de l'Empire. Iouxte la copie imprimee à Paris, [s.1.], 1648; au sujet du lieu de publication, voir la note précédente et RÉMY-LIMOUSIN, Traités internationaux, $\mathrm{n}^{\circ} 205$.

${ }^{60}$ Cependant, ce sera seulement sous Colbert que ce principe déclaré en 1537, après avoir été renforcé sous Séguier, s'établira durablement. Au sujet du dépôt légal, voir Henri-Jean MARTIN, article »Bibliothèque du Roi «, dans: BÉLY (dir.), Dictionnaire de l'Ancien Régime, p. 158-159.

61 Traité de la paix arresté a Osnabruk (1648).

62 Sommaire des articles de la paix generale entre l'Empire et la France, Paris, Imprimeurs et Libraires ordinaires du Roy, 1648. Cette édition a été réimprimée en 1649. Il s'agit d'ailleurs d'une véritable réimpression qui corrige seulement deux petites erreurs dans les articles 22 et 38 .

${ }^{63}$ Les Articles de la paix générale d'Allemagne (1648), publiés par le Bureau de Renaudot. ${ }^{64}$ Sommaire des articles de la paix generale, entre l'Empire et la France. Jouxte la copie imprimée à Paris, Metz, Pierre Colignon, Imprimeur juré du Roy et de ladite Ville. L'édition n'est pas datée. Cependant, le fait que toutes les autres éditions isolées des sommaires soient parues en 1648 ou en 1649 laisse supposer que celle-ci ait vu le jour en même temps ou, à la limite, peu de temps après. En effet, il fallait bien qu'il y eût un public qui s'intéressât à une telle édition; il est fort difficile d'imaginer un tel intérêt, qui faisait de la publication une affaire commerciale rentable, plus de deux ans après la signature des traités. Il nous semble bien que cette réflexion soit confirmée par le fait que l'édition de Metz répète la préface de la "Gazette«, qui fait allusion à la conclusion de la paix survenue très récemment. Comme la ville et l'évêché de Metz étaient directement concernés par le traité de Münster, il est de toute façon très probable qu'il y a été très vite publié. 
juré du roi et de la ville de Metz«. Tous les personnages responsables de ces publications se trouvaient donc favorisés par l'autorité. En fonction d'une politique qui devait compléter la censure, celle-ci s'était habituée, notamment depuis le ministère du cardinal de Richelieu, à accorder le monopole de la diffusion des actes officiels à des éditeurs-libraires et à des imprimeurs qu'elle voulait soutenir, et qui, à cet effet, obtinrent le titre d'»imprimeur [ou de >libraire`] du roi« «5. Cette politique de diriger l'édition moyennant le monopole et le privilège atteignit son sommet sous le règne personnel de Louis XIV.

En 1650 paraît un premier recueil qui regroupe les abrégés des deux traités, la paix entre l'Espagne et les Provinces-Unies du 30 janvier 1648 ainsi qu'un projet français pour le traité franco-espagnol qui avait été proposé aux Espagnols lors des négociations de Münster ${ }^{66}$. Ce recueil, bien qu'il se fonde toujours sur des abrégés, témoigne du désir de donner une idée complète sur tout ce qui avait été conclu dans ces traités fondamentaux. À la première information diffusée dans des éditions isolées et éparses, se substitue la recherche d'une vue d'ensemble tendance qui ne vient que de s'amorcer dans ce petit recueil in-12, mais qui s'accentuera et caractérisera les éditions des décennies suivantes. Celles-ci sont marquées par la parution d'un grand nombre de gros recueils in- $4^{\circ}$ ou in-folio qui ajoutent aux traités de Westphalie d'autres traités jugés nécessaires à leur compréhension, ou qui, inversement, ajoutent la paix de Westphalie aux éditions de traités postérieurs.

Outre les imprimés, les manuscrits servaient à faire connaître la teneur de la paix de Westphalie. Pour cette raison, l'on peut toujours trouver des copies latines et des traductions manuscrites françaises des traités. Dans la correspondance politique du Quai d'Orsay, c'est évidemment le latin, la langue des négociateurs et des ambassadeurs, qui prédomine ${ }^{67}$. On y conserve cependant une traduction manuscrite des articles du traité de Münster qui concernent la cession de l'Alsace ${ }^{68}$. Cette traduction témoigne du souci avec lequel on chercha à comprendre exactement les termes juridiques cruciaux qui devaient régler les modalités de la domi-

${ }^{65}$ Le titre d'»imprimeur du roi« n'est pourtant pas toujours très correct puisque, comme le rappelle Martin, ces prétendus imprimeurs n'étaient souvent que des libraires, qui faisaient à leur tour imprimer les ouvrages qu'ils vendaient par des ateliers typographiques parisiens, provinciaux et même étrangers, cf. MARTIN, Le livre français, p. 44.

66 Recveil de tovs les traitez accordez en l'assemblee generale, tenuë à Munster et Osnabruk en Vvestphalie, pour la paix de la chrestienté. Avec les noms et qualitez de tous les ambassadeurs \& plenipotentiaires qui ont assisté à ladite assemblee. Imprimé sur les originaux [s.l.] l'an de salut. 1650. Publié à Paris, selon le matériel typographique (cf. RÉMY-Limousin, Traités internationaux, $\mathrm{n}^{\circ}$ 190); résumés publiés dans la »Gazette de France« en 1648: IPM p. 13-60 (chapitre II), IPO p. 61-[99] et 100-143 (chapitres III à IIII). Le projet pour le traité franco-espagnol date très certainement du 25 janvier 1647 . Note manuscrite, à la fin de l'IPM et de l'IPO, dans l'exemplaire consulté à la BNF (M.14408): "A Paris, au Bureau d'adresse ... 1648« (ce sont les date et lieu de leur publication dans la »Gazette de France«).

${ }^{67}$ Des copies latines de l'IPM se trouvent par exemple: AE, CP All. 110 fol. 140-197, fol. 216-280 et fol. 281-328; copie latine de l'IPO: ibid., fol. 361-488.

68 Ibid., fol. 347-350: »Extraict du traicté de la paix d'Allemagne entre la France et l'Empire«; »1648. Allemagne, congrès de Munster«. 
nation française en Alsace, et en même temps définir les liens qui continueraient d'exister entre les ordres de cette province et l'Empire69. Mais, très certainement, d'autres copies ne circulaient pas seulement dans le monde des diplomates. Par exemple, le manuscrit 1228 de la bibliothèque municipale de Dijon contient, après les gravures des ambassadeurs qui ont pris part au congrès ${ }^{70}$, une copie manuscrite du sommaire de la paix de Münster ${ }^{71}$ ainsi qu'un très bref résumé de quelques stipulations du traité d'Osnabrück ${ }^{72}$. C'est un manuscrit qui, à plusieurs égards, fait preuve de particularités linguistiques surprenantes. On voit même des substantifs prendre des désinences verbales: »jugent« au lieu de »juges « (et non pas comme forme du verbe »juger«), »esglisent« au lieu d'»églises«. Ce manuscrit témoigne donc de la diffusion de quelques notions des traités de Westphalie dans des strates de la population qui n'appartenaient probablement pas aux cercles d'hommes politiques et de savants où leur connaissance est bien attestée. Soulignons, en revanche, que le possesseur de cette copie disposait apparemment des moyens d'acquérir les estampes des plénipotentiaires du congrès, qui se trouvent au début du manuscrit ${ }^{73}$. De plus, ce manuscrit, peu exact, est en même temps un témoin de la diffusion des traités recopiés à la main et de la déformation qu'ils pouvaient ainsi subir.

La traduction de la »Gazette « connut encore un épigone tardif, Pierre Louvet, qui inséra le sommaire du traité de Münster dans son livre »La France dans sa splendeur«, publié vingt-six ans après la fin de la guerre de Trente Ans, à Lyon ${ }^{74}$. Pierre Louvet ${ }^{75}$, que la $»$ Nouvelle biographie générale« qualifie d'»historien $\aleph^{76}$,

69 À ce sujet, voir LivET, L'intendance.

70 Bibliothèque municipale de Dijon, ms. 1228 fol. 1-36: »Portraits, noms, et qualitez des ambassadeurs [...]«, Paris, Baltazar Moncornet, s.d. À la Bibliothèque nationale, l'on trouve un volume qui renferme des "Traités de paix entre la France et la Maison d'Autriche«. Ce manuscrit contient exclusivement des pièces relatives aux traités de Westphalie, dont une copie du traité de Münster en latin avec, en marge du texte original, un abrégé des articles en français, qui reproduit, en partie littéralement, la traduction de la »Gazette«, BNF, F.fr. 20996 fol. 35-66'. Le texte est divisé en quatre-vingt-quatre articles, comme celui du sommaire français dans la »Gazette de France« qui servit de modèle à cet égard en France. Une autre copie des abrégés du préambule et des articles I à VI se retrouve sur un petit feuillet, au début du manuscrit, ibid., fol. 4 ( $\$ 1-5$ selon la numérotation actuelle).

71 Bibliothèque municipale de Dijon, ms. 1228 fol. 38-52.

72 Ibid., fol. 52'. Il n'y est question que du dédommagement de la Suède (en territoires et en argent); de plus, sur l'article de religion, on remarque seulement que »ceux de la confessions d'Ausbourg auront droit d'avoires autant de magistrats que les chatolicques, et [qu']on leurs rendra leurs esglisent et enseignes [sic]«.

${ }^{73}$ Selon la description de la bibliothèque municipale de Dijon (figurant au début de la photocopie du manuscrit qu'elle a bien voulu nous adresser), ce manuscrit datant du XVIIe siècle est composé de feuillets papier, avec une reliure de veau et l'ex-libris imprimé de »PetriLudov. Baudot«.

74 Pierre Louvet, La France dans sa splendeur, tant par la reunion de son ancien domaine qui étoit aliené. Que par les traitez de paix de Munster, des Pyrenées et d'Aix la Chappelle et par les conquestes de Sa Majesté, 2 vol., Lyon 1674, t. II, chapitre XXVII, p. 318-359.

$75 \mathrm{Cf}$. Biographie universelle ancienne et moderne, 85 vol., Paris 1811-1862, t. XXV, p. 278-279; Nouvelle Biographie générale depuis les temps les plus reculés jusqu'à nos jours, avec les renseignements bibliographiques et les sources à consulter, publiée par MM. 
n'était à vrai dire qu'un médecin de formation qui se mit à enseigner la rhétorique et les humanités parce qu'il n'avait pas pu obtenir de crédit pour exercer sa profession. Né en 1617 à Beauvais et mort probablement en 1684 en Provence, il ne se confond surtout pas avec l'avocat au parlement du même nom, qui fut aussi historien à Beauvais ${ }^{77}$. Issu d'une autre famille que ce dernier, il cultiva cependant une relation amicale avec l'avocat qui dura plusieurs années. Après divers postes d'enseignant, Louvet occupa finalement une chaire d'histoire et de géographie à Montpellier. Il consacra la plupart de ses auvres à l'histoire régionale de la France et publia notamment des »abrégés« de l'histoire de la Franche-Comté, du Languedoc, de la Provence et du Beaujolais. Sa »France dans sa splendeur « n'est qu'un "panégyrique de Louis XIV «78; il s' agit d'une description géographique, politique et historique de l'ancien domaine des rois de France et des provinces conquises par Louis le Grand, où Louvet poursuit le but d'illustrer les performances du roi. Ce n'est pas un livre très appréciable, qui souffre des mêmes faiblesses que les autres ouvrages de l'auteur 79 . Sa valeur pour l'historiographie se mesure déjà par le fait que Louvet n'a pas pris la peine d'offrir à son lecteur une édition complète du traité de Münster; l'abrégé qu'il en publie était assez bien fait pour fournir aux contemporains de la conclusion de ce traité une première idée de sa teneur, mais il n'a pas sa place dans l'ouvrage d'un historien sérieux ${ }^{80}$.

\subsection{Les traductions publiées de 1651 à 1754}

La publication de traités - nous venons de le dire - suppose l'existence de lecteurs qui s'intéressent aux affaires publiques. Cet intérêt, à en juger les publications, croît considérablement durant toute la deuxième moitié du XVIIe siècle. Le premier recueil qui donne une version intégrale du traité de Münster en langue française est, par rapport à ses successeurs, fort modeste. C'est un petit livre de 497 pages in-12 qui se limite aux seuls traités conclus par la France de 1621 à 1648. Publié pour la première fois en 1651 sous le titre de "Recueil des traictés de confédération et d'alliance, entre la couronne de France, et les princes et Estats estrangers « ${ }^{81}$, il contient non seulement des traités, mais aussi des lettres et d'au-

Firmin Didot Frères sous la direction de M. le $\mathrm{D}^{\mathrm{r}}$ Hoeffer, Paris 1857-1866 (réimpression, Copenhague 1963-1969), ici t. 32, col. 47-48; Alexandre CIORANESCU, Bibliographie de la littérature française du dix-septième siècle, 3 vol., Paris 1965-1966, t. II, p. 1308-1309.

76 Nouvelle Biographie générale, t. 32, col. 47. La »Biographie universelle« le traite d'»historien médiocre «, Biographie universelle, t. XXV, p. 278.

77 Né à Verderel en 1569 et mort à Beauvais le 23 décembre 1646, auteur d'un grand nombre d'ouvrages sur Beauvais, le Beauvaisis et sa noblesse, cf. CiORANESCU, Bibliographie de la littérature française du dix-septième siècle, t. II, p. 1309.

78 Biographie universelle, t. XXV, p. 279.

${ }^{79}$ On lui a surtout reproché que tout ce qu'il avait écrit était d'un style confus, mal digéré et par conséquent peu utile, Nouvelle Biographie Générale, t. 32, p. 48.

80 À part l'édition de Louvet, on notera aussi la toute dernière édition du résumé que la "Gazette « avait publié du traité d'Osnabrïck, dans un recueil de traités datant de 1698, publié à Luxembourg (voir ci-dessous).

${ }^{81}$ Recveil des traictés de confederation et d'alliance, entre la couronne de France, et les 
tres documents. En revanche, il n' offre aucune annotation ni explication, mais reproduit seulement les textes. Mario Toscano l'attribue à Jean-Jacques Chifflet ${ }^{82}$, ce qui paraît possible étant donné que Chifflet avait aussi édité, quelques années auparavant, le recueil pour les négociateurs espagnols ${ }^{83}$. Or, Toscano cite une édition de 1650 de ce recueil que nous ne sommes pas arrivés à retrouver ${ }^{84}$. En revanche, il ne connaît pas celle de $1651^{85}$. La question de savoir s'il y a une édition de 1650 ne parait pas cruciale. Les éditions de 1651 à 1672 reproduisant en gros une traduction identique, il est probable qu'une éventuelle édition antérieure offrait le même texte. La question n'est pourtant pas dépourvue de tout intérêt, car il s'agirait de la première édition intégrale du traité de Münster qui serait alors parue une année plus tôt que selon les résultats de nos recherches. Étant donné que Toscano cite la date à deux reprises ${ }^{86}$, il ne peut pas s' agir d'une simple erreur. Or, nous n'avons pu retrouver une quelconque trace d'une édition de 1650 , ni dans les grandes bibliographies, ni dans les catalogues des principales bibliothèques européennes et américaines. D'ailleurs, si ce livre avait déjà été réimprimé un an après la première édition, il aurait fallu qu'il eût été épuisé dans un délai extrêmement bref. De plus, l'»Avertissement « de l'imprimeur ${ }^{87}$ de l'édition de 1651 fait bien allusion à une édition parisienne publiée sous le titre de »Traictez de confédération ${ }^{88}$, mais à aucune autre édition antérieure, de sorte qu'il est probable qu'elle succède immédiatement au recueil de Paris. Même si l'existence d'une édition antérieure à 1651 n'est pas à écarter complètement, il semble par conséquent que Toscano se soit probablement trompé sur la date de publication; en revanche, on peut exclure que Toscano confonde ce recueil avec la collection parisienne de 1650 puisqu'il en fait une mention particulière. Le recueil a d'abord paru sine loco. Par la suite, il a été réédité en 1664, selon la datation discutable au frontis-

princes et Estats estrangers, depuis l'an M DC XXI jusques à present, avec quelques autres pieces appartenantes à l'histoire [par Jean-Jacques Chifflet?], s.l. [Amsterdam] 1651; sur cette édition, cf. RÉMY-LIMOUSIN, Traités internationaux, $\mathrm{n}^{\circ} \mathrm{R} 25$.

82 Toscano, History, p. 50; ID., Storia, p. 64.

83 [CHIFFLE], Recueil des traittez de paix, éditions de 1643, 1645 et 1669 (IPM: p. 407 455).

84 Celle-ci ne se confond pas avec les Traictez de confederation et d'alliance, entre la covronne de France, et les princes et Estats estrangers, s.l. 1650. Ce dernier recueil ne contient que les contrats de mariage de Marie de Médicis et d'Anne d'Autriche ainsi que les traités conclus par la France, surtout par Louis XIII, de 1628 à 1644; ouvrage publié à Paris, par la veuve de Th. Pépingué et par E. Maucroy, selon RÉMY-Limousin, Traités internationaux, $n^{\text {os }} \mathrm{R} 23$ et $\mathrm{R} 24$ (autre édition du même recueil, ibid., même année, même contenu et même matériel typographique, format et pagination différents).

85 Ni celle de 1668 .

86 Toscano, History, p. 50 et n. 9 (de même dans l'original italien: ID., Storia, p. 64 et n. 2). Cf. aussi GARDEN, Histoire, t. I, p. 276 (réimpression du "Discours préliminaire « publié par Georges-Frédéric de Martens en 1802).

${ }^{87}$ Recveil des traictés de confédération et d'alliance (1651), deux pages non numérotées au début du livre.

88 Il s'agit probablement de l'ouvrage suivant, mentionné ci-dessus: Traictez de confederation et d'alliance, entre la covronne de France, et les princes et estats estrangers, s.l. 1650. 
pice, puis en 1668 et en $1672^{89}$. Le titre de l'édition datée de 1664 indique comme lieu de publication l'atelier de Pierre Van Dyck à Amsterdam; or, une analyse typographique révélerait, selon Rémy-Limousin, l'origine belge du recueil90. De toute évidence, au moins les trois dernières éditions ont été tirées sur le même matériel typographique ${ }^{91}$. On peut donc faire l'hypothèse que ces trois dernières éditions du recueil au moins proviennent du même atelier. La première édition complète du traité de Münster en langue française serait donc parue aux Provinces-Unies ou aux Pays-Bas espagnols, et cette traduction intégrale aurait très certainement été réimprimée à trois reprises en Belgique. De toute façon, la traduction que ce recueil offre est très médiocre. Le traducteur ne semble pas avoir connu la traduction de la »Gazette«, du moins aucune influence de celle-ci n'est perceptible dans la version qu'il a établie du traité de Münster. Parmi les faiblesses de ce recueil, n'indiquons que les lacunes, qui y sont très nombreuses. À cela s'ajoute un certain nombre de fautes et de particularités terminologiques; ainsi, dans sa traduction du paragraphe 65 , le traducteur rendait le »votum decisivum " qui revenait aux villes libres de l'Empire à la diète par »voix deliberative«, tandis que les autres traducteurs traduisaient ce terme par »voix décisive «92.

En 1674, cette traduction a été reprise dans l'appendice des »Mémoires « sur les négociations de Münster, attribués par certains historiens au comte d'Avaux ${ }^{93}$; or,

${ }^{89}$ Recueil des traictés de confédération et d'alliance, édition datée $» 1664$ « et éditions de 1668 et de 1672.

90 Cf. RÉMY-Limousin, Traités internationaux, nos R 25 (1651), R 26 (1664 [i.e. 1667]), R 27 (1668) et $R 28$ (1672). Elle remarque à propos de la première édition qu'elle a été imprimée aux Pays-Bas (i.e. Provinces-Unies) ou en Belgique, d'après le matériel typographique, et à propos des éditions datant de [1667], 1668 et 1672 qu'elles ont été imprimées en Belgique, pour la même raison.

91 RÉmY-Limousin, ibid., est tout à fait formelle sur ce point quant aux éditions de [1667], 1668 et 1672 . Moins claire à cet égard, sa description de la première édition note pourtant un point en commun important (marque à la sphère au titre, qui correspond au trois éditions suivantes).

92 D'ailleurs, les deux traductions sont correctes (cf. notre chapitre sur la politique française au congrès de Westphalie). Il s'agit de la clause la plus importante concernant les villes impériales dans les traités de 1648. Les villes libres d'Empire obtinrent finalement gain de cause et eurent le droit de vote dans les diètes générales et particulières du Saint-Empire au même titre que les autres ordres, cf. OschMANN, APW III B 1/1, p. 20: $\gg$ Tam in universalibus vero quam particularibus diaetis liberis Imperii civitatibus non minus quam caeteris statibus Imperii competat votum decisivum«.

93 Memoires de Monsieur D.... Touchant les negociations du traité de paix fait à Munster en l'année mil six cens quarante-huit. Sur l'imprimé à Cologne [s.l.] 1674 (BNF: $\mathrm{Lg}^{6} .163$, note sur la page précédant le frontispice: »Par M. de Mesmes, C. ${ }^{\text {te }}$ d'Avaux «). Des éditions de ce livre seraient parues, outre à Cologne, à Paris et à Grenoble la même année, cf. Louis ANDRÉ, Les sources de l'histoire de France, XVIIe siècle (1610-1715) (vol. I-IV par É. BourgeOIS et L. ANDRÉ), 8 vol., Paris 1913-1935 (Les sources de l'histoire de France depuis les origines jusqu'en 1815, troisième partie; Manuels de bibliographie historique, 3), t. V, p. 226-227, notice 3724. Le traité de Münster est publié en annexe, avec une pagination et un frontispice particuliers: Instrvment, ov traité de paix, signé et seellé à Munster en Vvestphalie le 24 d'octobre 1648 par les ambassadeurs plenipotentiaires des Sacrées Majes- 
Tischer soutient que cette attribution est probablement incorrecte ${ }^{94}$. En effet, tous les exemplaires qu'elle a pu repérer, dans les bibliothèques françaises et allemandes, se réfèrent à une édition imprimée à Cologne. Or, Cologne était un lieu de publication fictif très à la mode, en particulier depuis 1663; il est donc possible que la référence à Cologne ait servi à tromper les autorités sur l'origine de l'ouvrage, publié sans privilège. En ce qui concerne l'édition qui aurait été publiée à Grenoble, d'après certains auteurs, il est à noter que la vignette ornant le frontispice des »Mémoires « ressemble effectivement à celle d'un imprimeur de cette ville, mais Tischer constate qu'il s'agit d'une imitation; il n'est pas exclu que cette similitude ait semé la confusion parmi les bibliographes. De toute façon, Tischer n'a retrouvé qu'une seule édition de l'ouvrage, se référant à une édition antérieure qui aurait été imprimée à Cologne, ornée de la vignette imitée ${ }^{95}$. Ce petit volume ne constitue pas des mémoires proprement dites, mais plutôt une relation des négociations et une apologie de la politique religieuse de la France ${ }^{96}$. Cependant, il s'agit plutôt d'une justification de la politique du comte d'Avaux en particulier que d'une apologie de la politique confessionnelle de la délégation française en général, comme le prétend Louis André97. Tombé en disgrâce, du moins temporaire ${ }^{98}$, d'Avaux, pour sa part, fut révoqué du congrès le 13 mars 1648 , quitta Münster le 18 avril et conçut le projet de publier une justification de sa politique. Mis en garde par ses amis, il y renonça ${ }^{99}$. C'est grâce aux recherches minutieuses d'Anuschka Tischer qu'on connaît mieux, à l'heure actuelle, l'histoire et les différentes versions manuscrites de ce texte. S'il semble peu probable que son auteur soit le comte d'Avaux, l'attribution du texte à son collègue Abel Servien suscite

tez Imperiale et Tres Chrêtienne, \& des deputez extraordinaires, électeurs, princes \& estats du Sacré Empire Romain, s.1. 1674.

94 Cf. TISCHER, Französische Diplomatie, p. 424-438.

95 Cf. ibid., p. 424-425.

96 Selon l'auteur, Ia guerre de Trente Ans "a esté purement une guerre d'Estat \& non de Religion, la France ne s'est jamais entremise dans les griefs des Protestans qu'elle a laissé decider entre les Ambassadeurs Imperiaux \& Suedois«, Memoires de Monsieur D., p. 21. En Allemagne, l'objectif politique de la France était d'»empécher qu'il n'y eût changement dans la forme de l'Empire«, ibid., p. 17.

97 Cf. ANDRE, Les sources, t. V, p. 226-227, notice 3724.

98 TISCHER soutient qu'il ne s'agissait pas d'une disgrâce stricto sensu, puisque d'Avaux ne fut pas officiellement blâmé et que quelques mois après son retour, le 15 décembre 1648 , il fut reçu par Anne d'Autriche, étant même autorisé à exercer de nouveau ses fonctions ministérielles de surintendant des finances en France et de membre du Conseil d'en haut à partir de 1649; cependant sa révocation était due au fait que Mazarin désapprouvait sa politique à Münster, et on lui interdit temporairement de se présenter à Paris. Outre la mésentente qui existait entre d'Avaux d'un côté et Servien et Mazarin de l'autre, le départ de Longueville et certaines prises de position du comte, notamment à l'égard de la Lorraine, expliquent cette décision. Cf. EAD., Französische Diplomatie, p. 113-118 et p. 171-180. Absente des discussions au moment où Mazarin prit la décision de révoquer d'Avaux, la question de la politique confessionnelle de la France avait pourtant largement alimenté les différends entre d'Avaux et Mazarin en 1647.

${ }^{99} \mathrm{Cf}$. Dickmann, Der Westfälische Frieden, p. 479. Dickmann ne cite toutefois pas de preuves irréfutables à ce propos (cf. les notes ibid., p. 575). 
aussi les réserves de Tischer ${ }^{100}$; il est vrai que Servien n'a pas partagé les opinions concernant la politique religieuse de la France qui sont défendues dans ces »Mémoires $\ll 101$.

Parmi les grands libraires parisiens du dernier tiers du XVII ${ }^{e}$ siècle, Frédéric Léonard, qui s'autodésignait par "Federic Leonard«, était un des plus riches et des plus illustres. C'est lui qui édita la grande collection des auteurs latins à l'usage du dauphin, connue encore aujourd'hui pour sa grande qualité102. Fils d'un libraire de nouveautés de Bruxelles en relation avec la petite cour française autour de Marie de Médicis lors de son exil, il fit son apprentissage chez les Plantin-Moretus, à Anvers, et vint à Paris dans la suite de la duchesse d'Orléans, belle-sœur de Louis XIII, en 1643. C'est là que Léonard, qui »pratiquait l'art de s'immiscer dans la faveur des grands «103, fit une irrésistible ascension. Malgré son origine étrangère, il arriva à se faire recevoir maître (1653) et racheta les fonds de deux libraires importants, Jean Petitpas et Sébastien II Huré. Avec l'acquisition de ce dernier fonds, il eut encore la charge d'imprimeur du roi (1661). Multipliant les ouvrages de spiritualité, il fut nommé imprimeur du clergé de France et de divers ordres religieux. Le roi le nomma seul imprimeur pour la guerre, les monnaies et les finances. Ayant aussi obtenu le poste d'imprimeur du parlement, Léonard s'était ainsi assuré le soutien et la faveur de tous les pouvoirs en place, politiques et ecclésiastiques ${ }^{104}$. Usant de ses privilèges extraordinaires, il amassa une fortune immense; quand il se retira des affaires, à partir de 1690 , il possédait, avec son fils, le château de Bois-Préau près de Malmaison. La fortune de son fils, Frédéric II Léonard, s'élevait à presque un million de livres en 1709. L'inventaire après décès du 15 octobre 1706 fait état d'une dette du roi et de ses ministres envers Léonard qui dépasse largement les trente mille livres ${ }^{105}$; on peut mesurer par là l'enjeu commercial que représentait alors la publication de documents officiels.

100 Cf. Tischer, Französische Diplomatie, p. 424-438. Elle conclut que l'auteur de ces mémoires n'a pas forcément participé au congrès de Westphalie; en particulier, elle ne pense pas qu'il s'agisse du comte d'Avaux ni de Servien comme l'ont suggéré certains biographes de ce demier. Elle prétend (ibid., p. 426, n. 9) que nous avons attribué ces mémoires au comte d'Avaux; or, comme nous avons mis son nom entre parenthèses, nous ne pensons pas que cette critique soit justifiée, cf. BRAUN, Traductions, p. 135, n. 22.

101 C'est l'une des raisons pour lesquelles elle exclut que Servien soit leur auteur, cf. Tischer, Französische Diplomatie, p. 424 438, en particulier p. 438.

102 La particularité du prénom s'explique par l'admiration que Léonard avait pour Fédéric Morel, grand imprimeur humaniste, qui avait mis ses capacités au service de la Contre-Réforme. Il n'y a aucun article biographique satisfaisant concernant Léonard. On se reportera à MARTIN, Le livre français, t. II, p. 717-719 et passim (cf. index, t. II p. 1037); voir aussi ID., chapitre »La prééminence de la librairie parisienne«, dans: CHARTIER, MARTIN (dir.), Histoire de l'édition française, t. II, p. 331-357, ici surtout la première annexe intitulée »Un protégé du roi: Frédéric Léonard«, p. 354-355.

103 ID., Livre, pouvoirs et société, t. II, p. 718.

104 Et ceci malgré certains choix qui pouvaient paraître contestables; ainsi conserva-t-il d'étroites relations avec son pays d'origine (pour les relations commerciales de Léonard avec l'étranger, cf. ibid., appendice, carte 8).

105 Ibid., p. 719, à la p. 675, n. 18 et à la p. 722, Martin avance même le chiffre de cinquante mille livres. ID., La prééminence, p. 354, parle de trente-trois mille livres de dettes du roi 
En 1678, Léonard publia un premier recueil susceptible d'intéresser l'historien des relations internationales. Sous le titre de »Recueil de tous les actes, memoires, et lettres, qui ont servi pour la negotiation de la paix, avec les traitez qui ont esté conclus à Nimegue«, il regroupa un certain nombre de pièces diplomatiques et de traités qui, en grande partie, avaient déjà été publiés séparément, aux armes de France ou à l'emblème du soleil ${ }^{106}$. Ce premier recueil montre déjà toutes les caractéristiques de ceux qui devaient suivre: les différentes pièces, qu'on pouvait pour des raisons commerciales aussi acheter seules, sont simplement reliées dans un volume sans pagination continue. Toutes les pièces gardent leur propre frontispice. On a ajouté un frontispice pour l'ensemble. Il n'y a aucun commentaire; l'édition n'offre que le texte seul des documents. Les traités conclus par l'Empire en 1679 , en version bilingue ${ }^{107}$, ont été ajoutés postérieurement, mais le frontispice du recueil porte toujours la date de 1678. Malgré ces inconvénients, le recueil connut un grand succès qui encouragea Léonard à suivre cette voie. En effet, il avait obtenu, le 18 août 1678 , le privilège »d'imprimer seul pendant vingt ans« les traités internationaux »qui ont esté faits cy-devant en Latin, en François ou autre Langue«. Le privilège lui attribua en outre les droits de traduction et précisa même qu'il s'agissait aussi bien du droit de publier les traités séparément que de $» 1$ es mettre en Recueil $\ll 108$. Pourvu de ces prérogatives exorbitantes ${ }^{109}$, dont furent pourtant exclus les privilèges déjà existants, Léonard se mit à publier, dès 1679, des recueils qui contenaient non seulement les traités de Nimègue, mais aussi des traités antérieurs, des lettres et des mémoires pour que l'on pût comprendre leur genèse. Il commença par le traité de Münster, qu'il publia aussi bien dans ces recueils que séparément, avec les armoiries royales (armes de France et couronne royale) au frontispice. C'est le texte seul du traité, bilingue, en latin et en français, sur deux colonnes. Il a été inséré dans au moins quatre recueils: deux recueils parus en 1680, quoique datés de 1679 (le premier contient des documents de 1648 à février $1679^{110}$, le second des pièces de la période qui va de 1648 à sep-

pour des impressions officielles, et attribue à Léonard père, en 1690, une fortune s'élevant à un million de livres.

106 [Frédéric LÉONARD], Recueil de tous les actes, memoires, et lettres, qui ont servi pour la negotiation de la paix, avec les traitez qui ont esté conclus à Nimegue, Paris 1678 [i.e. 1679]; cf. RémY-Limousin, Traités internationaux, $n^{\circ} \mathrm{R} 36$. Toutefois, ce recueil ne contient pas les traités de Westphalie.

107 Latin/français sur deux colonnes.

$108 \gg$ Extrait du Privilege du Roy«, dans: [Frédéric LÉONARD], Traité de paix entre l’Empereur, et le Roy tres-chrestien, et les électeurs, princes, et états du Saint Empire, conclu à Munster en Westphalie le 24. octobre 1648, Paris 1679, p. 64.

109 Selon DUMONT, Léonard aurait de même joui de la faveur de "quelques Ministres « qui lui auraient communiqué des centaines de pièces; ID., Corps universel, t. I, p. V, art. XXVII. 110 [Frédéric LÉONARD], Recueil de divers traitez conclus entre les potentats de l'Europe, et de tous les memoires et lettres qui ont servi a la negociation de la paix de Nimégue, Paris 1679 [i.e. 1680]. Recueil de 58 documents en 1 vol. in- $4^{\circ}$ sans pagination continue; la table des matières ( 4 p. non paginées, placée au début du volume) est datée de 1680 . Le frontispice du recueil est orné de trois lys, timbrés de la couronne royale. Pour ce recueil, cf. RÉMY-LI- 
tembre $1679^{111}$ ), et deux recueils publiés respectivement en $1683^{112}$ et en 1693113. Ce sont des volumes factices regroupant plusieurs brochures parues isolément chez Léonard et divers libraires parisiens, pour l'ensemble desquelles on a imprimé une »table de pièces « générale. Ils réunissent, comme on l'a dit, des documents diplomatiques datant de la période 1648-1679 essentiellement. La plupart des textes sont en français, mais il y en a aussi en espagnol, en néerlandais, en latin et, comme par exemple le traité de Münster, en version bilingue ${ }^{114}$. Deux observations s'imposent: premièrement, le fait qu'on commence généralement par le traité de Münster montre que l'année 1648 fut véritablement considérée comme l'aube d'une nouvelle époque dans les relations internationales en Europe; deuxièmement, le traité d'Osnabrück reste pourtant le grand absent, l'intérêt va exclusivement vers le traité conclu par la France elle-même. Le recueil de 1693 fait exception, car on y publie aussi le traité d'Osnabrück, dans la traduction de Jean Heiss $^{115}$, et avec une assez grande fidélité. S'il s'agit toujours d'une collection factice de pièces imprimées à différentes époques (pour lesquelles on a composé des titres, des tables et des notices préliminaires), le recueil dépasse largement ses prédécesseurs par le nombre de pièces qu'il contient: environ neuf cents traités, sans compter les autres documents, dans six volumes in-4 $4^{\circ}$. Malgré tous les inconvénients typiques des publications de Léonard, cette collection était la plus grande entreprise de ce genre jamais réalisée jusqu'alors et devait servir de source aux compilations suivantes, tel le »Grand Recueil« de $1700^{116}$. La richesse en docu-

MOUSIN, Traités internationaux, $n^{\circ} \mathbf{R} 37$ (qui n'a pas remarqué la datation de la table des matières). Le traité de Münster est le document 58 dans ce volume.

111 Ce recueil porte le même titre que le précédent; il contient 45 documents en 1 vol. in-4\%, pagination multiple, ibid., 1679 [i.e. 1680]. Nous n'avons pas vu cette édition; l'exemplaire de la BNF est en effet classé »hors d'usage«, depuis de nombreuses années. Cf. RÉmY-LIMOUSIN, Traités internationaux, $n^{\circ} \mathrm{R} 38$ (d'après cette description, l'édition est datée $» 1679$ « mais porte, au frontispice, un portrait de Louis XIV daté de $» 1680$ «).

112 [Frédéric LÉONARD], Recueil de tous les traités modernes conclus entre les potentats de l'Europe: De tous les mémoires qui ont servi a faire la paix de Nimégue: Et de tous les arrests de la Chambre roiale de Mets, relatifs aux traités de Nimégue \& de Munster, Paris 1683 . Recueil de 70 documents en 1 vol. in-folio sans pagination continue. Cf. RÉmY-Limousin, Traités internationaux, $n^{\circ} \mathrm{R} 39$. Le traité de Münster est le document 70 dans ce volume.

113 Frédéric LÉONARD, Recueil des traitez de paix, de treve, de neutralité, de confederation, d'alliance, et de commerce, faits par les Rois de France, avec tous les princes, et potentats de l'Europe, et autres, depuis pres de trois siecles. En six tomes. Assemblé, mis en ordre, \& imprimé par Frederic Leonard, premier imprimeur du Roi, \& de Monseigneur le Daufin, Paris 1693. Ce recueil contient non seulement l'IPM, mais aussi une traduction française de l'IPO (t. III). Les différentes pièces contenues dans ce recueil sans pagination continue ne sont pas numérotées, mais disposées par ordre chronologique. Cf. RÉMY-LIMousIN, Traités internationaux, $\mathrm{n}^{\circ} \mathrm{R} 10$.

114 Voir ibid., aux numéros indiqués ci-dessus, pour une description du contenu des recueils. 115 Au sujet de cette traduction appelée à devenir canonique en France, voir ci-dessous.

116 Recueil des traitez de paix, de trêve, de neutralité [...], \&c. Faits entre les Empereurs, Rois, républiques, princes, \& autres puissances de l'Europe, $\&$ des autres parties du monde. Depuis la naissance de Jesus-Christ jusqu'à présent [ou Grand Recueil de Hollande, par Jacques Bernard], 4 vol., Amsterdam, La Haye 1700. Traités de Westphalie: t. III; paix de 
ments de cet ouvrage est due au fait que Léonard avait accès aux archives royales, et pouvait y puiser pour ses éditions ${ }^{117}$ qui avaient un caractère officiel. Si le recueil de 1693 fut publié pour satisfaire à un plus large public de "gens cultivés«, celui de 1683 , de toute évidence, avait été commandé par le gouvernement pour répondre aux critiques provoquées par la politique des chambres de réunion de Louis XIV118.

Or, la traduction de la paix de Münster chez Léonard ${ }^{119}$ est tellement erronée qu'on ne saurait par où commencer si on voulait la commenter. De toute évidence, on a essayé de corriger la traduction de 1651 qui est à la base de celle de Léonard, en recourant au sommaire de la »Gazette«. Mais le seul résultat de ces >corrections ‘ fut de faire d'une version médiocre une traduction complètement ratée. Au dépôt des Affaires étrangères, on la qualifiait d'»infidèle en beaucoup de points essentiels, et entièrement défectueuse « 120 .

La traduction appelée à faire autorité, de sa première publication, en 1684, à nos jours, est celle de Jean Heiss. Selon nos recherches, après lui, personne n'a plus jugé opportun d'établir une nouvelle traduction du traité de Münster ${ }^{121}$ ni de reprendre celles qui avaient été faites avant Heiss ${ }^{122}$. Sa traduction du traité d'Osnabrück est la première publiée depuis les abrégés de 1648-1650, et en même temps la première édition intégrale en français. Cette traduction est d'une très

Münster: p. 528-540 (en latin); paix d'Osnabrück: p. 541-563 (en français). Cf. RÉMY-LIMOUSIN, Traités internationaux, $\mathrm{n}^{\circ} \mathrm{R} 3$.

${ }^{117}$ Le jugement que Toscano en fait est pertinent; il relève que les éditions de Léonard obéirent à des choix plutôt commerciaux ou répondirent peut-être à des pressions politiques. De toute façon, les critères selon lesquels il choisit les documents qui devaient être insérés dans ses recueils ne sont pas clairs, et l'organisation des volumes paraît discutable, puisque Léonard chercha surtout à publier très rapidement le plus grand nombre de documents possible; cependant, Toscano met en relief que Léonard pouvait utiliser le trésor des chartes, la Bibliothèque royale et les archives des chambres des comptes de Paris, de Lille, de Nantes, etc. Ainsi Toscano conclut-il que Léonard a porté à notre connaissance un très grand nombre de documents inédits, mais les chercheurs se réfèrent aujourd'hui aux recueils publiés par la suite par les éditeurs néerlandais, qui avaient critiqué Léonard tout en puisant dans ses recueils, puisque ces collections hollandaises, qui datent du XVIII ${ }^{e}$ siècle, ont un plan plus clair et sont donc plus faciles à consulter, cf. TOSCANO, History, p. 53; ID., Storia, p. 69.

118 Selon ID., History, p. 53; ID., Storia, p. 68: »Le recueil avait un caractère officieux évident et sans aucun doute il avait été commandé à Léonard pour répondre aux réactions provoquées dans toute l'Europe par la politique des `Chambres de Réunion` instaurées par Louis XIV « (»La raccolta aveva un evidente carattere ufficioso e con tutta probabilità era stata commissionata a Léonard per ripsondere alle reazioni provocate in tutta l'Europa dalla politica delle >Camere di Riunione < inaugurata da Luigi XIV $\ll$ ).

119 Ce n'est certainement pas Léonard lui-même qui a fait la traduction, mais, pour simplifier, nous la qualifierons par la suite de »traduction de Léonard«.

120 Note ajoutée sur l'imprimé de cette traduction: AE, CP All. 122 fol. 548; aussi cité par VAST, Les grands traités, p. 8.

121 La seule exception, à notre connaissance, est une version manuscrite faite au dépôt des Affaires étrangères, dans la deuxième moitié du XVIIIe siècle. Voir ci-dessous.

122 À l'exception de Léonard, qui continua à faire réimprimer sa version du traité de Münster, avec le texte latin, dans une édition bilingue, au moins jusqu'en 1693, cf. LEONARD, Recueil des traitez de paix (1693). 
grande qualité même si stylistiquement elle paraît parfois un peu maladroite. Mais c'est parce qu'elle suit scrupuleusement l'original latin et cherche à le transposer le plus fidèlement possible en français. Ceci est valable pour la syntaxe aussi bien que pour les termes juridiques. Le jugement sévère qu'y portera quelque soixantequinze ans plus tard Scheid, lui aussi traducteur de la paix d'Osnabrück ${ }^{123}$, n'est aucunement justifié. Certes, il y a toujours quelques lacunes qui ont échappé à Heiss lorsqu'il collationnait la traduction de Léonard, dont il se servait en établissant la sienne. Il faut cependant bien voir que Heiss partait presque ex nihilo; il a fait des ruines que lui avait laissées Léonard une maison bien solide. Même si Scheid en fera un château par la suite, cela ne doit point remettre en question son mérite. L'ouvrage de Heiss a un très grand avantage: celui d'offrir une interprétation complète des traités. En effet, les traités de Westphalie, comme d'autres documents (tels que la Bulle d'or, autre loi fondamentale de l'Empire), y sont ajoutés en tant que pièces justificatives (»servant de preuves à l'Histoire de l'Empire«, selon l'expression de la seconde édition). Heiss composa son »Histoire $\ll$, qui est centrée sur l'évolution du droit public, en deux parties, dont la première traite de l'Empire avant 1648 et la deuxième des changements apportés par les traités de Westphalie. L'explication de ces traités à un public francophone est donc l'objectif même de Heiss. La »Nouvelle Biographie générale« estime que »le style de cet ouvrage ne trahit pas l'origine [allemande] de l'auteur, qui a su donner à ses contemporains le premier résumé lucide de l'histoire si embrouillée de l'Empire«124.

Heiss est d'ailleurs un personnage dont la biographie est très intéressante pour l'histoire de la connaissance du droit public allemand en France; même s'il n'était pas de souche alsacienne, comme on l'avait supposé, mais originaire de Clèves, sa biographie reste typique des propagateurs de la connaissance du ius publicum Imperii en France, car il menait une vie entre deux cultures, française et allemande, et jouait un rôle de médiateur entre les deux civilisations ${ }^{125}$. Naturalisé français en

\section{Cf. ScheID, Traité sistématique, t. I, p. [*7], et t. IV, p. 149-299.}

124 Nouvelle Biographie générale 23 (1861), col. 804-805. Presque tous les historiens anciens et modernes estiment que Heiss est très probablement, voire très certainement identique à l'auteur de l'»Histoire de l'Empire«; pour un jugement différent, cf. chapitre IX (n. 120).

125 Sur Heiss: Biographie universelle, t. XIX (1817), p. 589-590; Nouvelle Biographie générale, t. 23 (1861), col. 804-805; Jean-Yves MARюTTE, article "Heiss, Jean «, dans: NDBA, t. 16 (1990), p. 1492; Archives biographiques françaises, Imicrofiches, parties I (première publication par Susan BRADLEY en 1989-1991), II (première publication en 1998) et III, avec un index cumulé imprimé, en 7 vol.], compilé par Tommaso NAPPO, Munich ${ }^{3} 2004$, ici I 508, $128-129 ; 1061,243 ;$ II 335, 22. Malheureusement, la plupart des articles anciens sont parsemés d'erreurs, notamment dans la mesure où ils attribuent une origine alsacienne à Heiss; toutefois, son ascendance alsacienne est remise en cause par Mariotte. Une contribution récente, qui tient compte de ses lettres de naturalisation et d'autres documents d'archives, n'a malheureusement pas été publiée; Jörg ULBERT, Le »double emploi« dans la diplomatie française (1648-1789): l'exemple de Jean Heiss. dans: Festgabe für Herrn Prof. Dr. Dr. h.c. Klaus Malettke zum 60. Geburtstag überreicht von seinen Schülern, dact. [Marbourg 1996]. 
$1643^{126}$, il garda un contact étroit avec son pays d'origine; ne se contentant pas de représenter, à Paris, divers princes allemands, il se vit aussi confier des missions diplomatiques dans l'Empire au nom de la France. C'est une biographie dont les traits essentiels se retrouvent dans la vie d'autres historiens et publicistes du XVII ${ }^{e}$ siècle qui étaient au service de la France dans les relations avec l'Allemagne $^{127}$ : d'origine allemande ou très souvent alsacienne, pourvus d'une bonne formation juridique, disposant notamment d'une excellente connaissance du droit allemand, successivement au service de princes allemands et de la cour de France, souvent chargés de missions diplomatiques en France avant de changer de maître. Si les contemporains de Heiss n'ont pas toujours porté un jugement positif sur l'»Histoire de l'Empire «128, les nombreuses rééditions que connut celle-ci témoignent cependant de son grand succès et de la confiance qu'y portaient juristes et historiens. Ce n'est qu'au milieu du XVIII' siècle que Heiss perdra de son importance et que Scheid portera un jugement très sévère sur son œuvre ${ }^{129}$. Par ailleurs, une biographie récente a encore loué son livre ${ }^{130}$.

De toute façon, les équivoques terminologiques que l'on peut constater avant 1684 cessèrent au moment où Heiss publia son »Histoire de l'Empire «, fût-ce par son influence ou par une évolution générale qui était sur le point de s'achever. Heiss a puisé dans le vocabulaire que la langue française avait déjà développé, mais il y a aussi ajouté un concept: en effet, c'est dans sa traduction du traité de Münster que le terme »immédiateté « (faisant partie de l'expression »immediateté à l'égard de l'Empire Romain «) est attesté pour la première fois en français ${ }^{131}$, du

126 Ses lettres de naturalisation qui datent du mois de décembre 1643 se trouvent aux Archives nationales, $K 173 n^{\circ} 67$. Elles précisent que Heiss est originaire de Clèves.

127 Voir le chapitre IX.

128 C'est du moins l'opinion formulée par Weiss, auteur de l'article biographique paru dans la Biographie universelle, t. XIX (1817), p. 589-590, ici p. 590. Il l'impute à un "jugement beaucoup trop sévère de cet ouvrage « qu'y aurait porté Lenglet et qui aurait été repris par les compilateurs venus après celui-ci. Or, le jugement de Lenglet, au moins dans la première édition de sa »Méthode«, est très positif. Il estime que le livre est écrit »avec beaucoup d'exactitude et de brieveté « et »d'une maniere raisonnable, quoique fort abregée «; [Nicolas abbé LENGLET-DUFRESNOY], Methode pour etudier l'histoire [...], 2 vol., Paris 1713, ici t. I, p. 132 et 143. Si ce jugement a été modifié dans les éditions suivantes, majoritairement dues à d'autres auteurs, elle ne correspond très certainement pas au jugement original de Lenglet; pour les autres éditions, cf. Alexandre CIORANESCU, Bibliographie de la littérature française du dix-huitième siècle, 3 vol., Paris 1969, ici t. II, p. 1092-1094.

129 SCHEID, Traité sistématique, t. I, p. *.

130 MARIOTTE, Heiss: »ouvrage remarquable par sa clarté et reproduisant de nombreux documents «.

131 Cf. [Jean] Heiss, Histoire de l'Empire [... ], 3 vol., La Haye 1685, t. III, p. 38, traduction du $\S 87$ du traité de Münster confirmant aux états de l'Empire en Alsace le respect de leur Reichsstandschaft par le roi de France; la traduction de la première édition (1684) est identique. À noter que si Heiss recourt aussi à cette expression dans son exposé, sa terminologie est encore chancelante: en effet, il continue à employer des périphrases traditionnelles en traduisant d'autres paragraphes. Pourtant, le concept »immédiateté « s'impose. Au cours des années 1770 , Jean-Jacques de Zabern, dernier traducteur de la paix de Münster (voir ci-dessous), l'utilise dans sa traduction du § 87, cf. AE, MD All. 41 fol. 90, 91-121, ici fol. 109'. 
moins si l'on en croit le "Trésor de la langue française«, qui se fonde sur le dépouillement des livres imprimés ${ }^{132}$. Nous pouvons dater plus précisément son apparition dans la langue française. En effet, il est employé dans plusieurs manuscrits de la Bibliothèque nationale et du Quai d'Orsay antérieurs à la première édition de Heiss; d'autres formes comme "immédieté« au singulier et »immédiatez « au pluriel accompagnent ses toutes premières attestations ${ }^{133}$. Selon nos recherches, il est apparu, ou a réussi à s'implanter durablement en français, au début des années 1660 . Il est intéressant de voir que ce mot s'est maintenu en français, bien qu'il ne soit très certainement pas l'un des plus courants ou des plus beaux de la langue de Molière; d'autres termes qui ont servi à traduire des concepts du droit public allemand n'y ont pas réussi, comme nous le verrons dans les chapitres suivants. Il est donc à noter que le terme »immédiateté « fut introduit en français pour traduire le mot allemand "Reichsunmittelbarkeit « et le concept latin "immedietas erga imperium romanum «, avant d'acquérir le sens général de »qualité de ce qui est immédiat«, qu'il revêt à l'heure actuelle.

L'»Histoire de 1'Empire» fut rééditée en $1685^{134}, 1694^{135}, 1711^{136}, 1715^{137}$,

132 Trésor de la langue française, t. 9, p. 1173 s.v. »immédiateté«; en reprenant la définition d'Émile Littré, le »Trésor « note que ce terme a désigné initialement, dans le droit féodal, un noble ou un fief »qui relève directement du roi ou de l'empereur, sans reconnaître d'autre souverain que lui«, en particulier la qualité ou le »privilège d'un noble ou d'un fief immédiat de l'Empire«; alléguant l'»Histoire de l'Empire« de Heiss comme première attestation de ce mot, le "Trésor « note que depuis 1721, il désigne la qualité de ce qui est immédiat, sans intermédiaire. Les prédécesseurs de Heiss avaient encore utilisé exclusivement des groupes nominaux comme "possession immédiate (Gazette [de France], no 174 [1648], p. 1554: les états de l'Empire dont les territoires sont situés en Alsace »demeurent dans la liberté \& possession immédiate dont ils ont joüi jusques à présent à l'égard de l'Empire «), »recognoissance immédiate « (dans la traduction manuscrite AE, CP All. 110 fol. 347-350, ici fol. 349': le Roi Très Chrétien doit laisser les états de l'Empire »dans la mesme liberté et possession de recognoissance immédiate vers l'Empire Romain « qu'ils ont eu auparavant) ou des périphrases plus complexes (cf. par exemple LÉONARD, Traité de paix [1679], p. 47: le roi de France doit laisser les états de l'Empire »dans le droit dont elles [sic] ont joüi jusqu'ici de relever immédiatement du Saint Empire«).

133 Cf. BNF, F.fr. 12122 p. 13: ce manuscrit a probablement été rédigé vers 1679; AE, MD Alsace 19 fol. 49, 64, 71 et 210': ce manuscrit date des années 1661 à 1663 .

134 HeIss, Histoire de l'Empire (1685); traités de Westphalie, t. III, IPM: p. 3-53; IPO: p. 54-153. Dans l'édition de 1684, les traités de Westphalie se trouvent à la fin du t. II (pagination particulière), IPM: p. III-XLVII; IPO: p. XLIX-CXXVI.

135 ID., Histoire de l'Empire, [...]. Troisiéme édition augmentée de plusieurs remarques, 3 vol., La Haye 1694. Cette édition est divisée en trois parties; titre du t. III: Divers traitez de paix conclus et signez a Munster et a Osnabrug en Westphalie le 24. octobre 1648. Comme aussi ceux qui furent faits a Nuremberg en execution des premiers, touchant la satisfaction des milices [...]. Servant de preuves à l'histoire de l'Empire, ibid. 1694; IPM: t. III, p. 3-53; IPO: ibid., p. 54-153.

136 ID., Histoire de l'Empire, [...]. Nouvelle édition continuée jusques à present, \& augmentée de plusieurs remarques [titre selon les t. I, III et IV]; Nouvelle édition continuée jusques à la mort de l'Empereur Joseph [d'après le t. II]; Nouvelle édition augmentée de plusieurs pieces, \& de plusieurs remarques [d'après le t. V], [par H. Bourgeois du Chastenet], 5 vol., Paris 1711; IPM: t. V, p. 118-176; IPO: t. V, p. 177-282.

137 ID., Histoire de l'Empire, [. . . ]. Nouvelle édition, continuée jusques à présent, \& augmen- 
1731,1733 (on note deux éditions différentes respectivement pour les années $1731^{138}$ et $1733^{139}$ ). En outre, elle a été traduite en anglais, entre 1727 et $1729^{140}$, en $1730^{141}$ et en $1731^{142}$. Les éditions françaises parurent en partie à Paris, en partie à Amsterdam ou à La Haye. L'édition de 1694, à la différence de celles de 1684 et 1685, est divisée en trois parties, la troisième partie étant constituée des »pièces justificatives « ${ }^{143}$. Les éditions du XVIII ${ }^{\mathrm{e}}$ siècle ont été complétées et continuées par deux personnages autrement mal connus. Celle de 1711 a été continuée par H. Bourgeois du Chastenet (ou Duchastenet) ${ }^{144}$ jusqu'à l'année de sa publication. Au sujet de ce personnage, on sait seulement qu'il vécut en France dans la première moitié du XVIII' siècle $^{145}$, qu'il exerca la profession de jurisconsulte et

tée de plusieurs remarques \& de nouvelles pieces authentiques [par H. Bourgeois du Chastenet]. Imprimée suivant la copie de Paris, 4 vol., La Haye 1715; IPM: t. IV, p. 62-123; IPO: ibid., p. 124-232.

138 ID., Histoire de l'Empire, [...]. Nouvelle édition. Augmentée de notes historiques \& politiques, \& continuée jusques à present. Par Monsieur V.G.J.D.G.S. [Vogel, grand juge des gardes suisses], 8 t. en 10 vol. in-8 ${ }^{\circ}$, Paris 1731; IPM: t. VII, vol. 9, p. 137-208; IPO: ibid., p. 209-333; autre tirage: Nouvelle édition [...] Dediée à S.A.S. Monseigneur le duc du Maine [Louis-Auguste de Bourbon], prince souverain de Dombes, 3 vol. in-40, ibid. 1731; IPM: t. III, seconde partie (pagination à part), p. 47-68 [annotations dans l'exemplaire du Quai d'Orsay (cote 9 Az 38), par exemple p. 58-59]; IPO: ibid., p. 69-106.

139 ID., Histoire de l'Empire, [...]. Nouvelle édition, augmentée d'un discours préliminaire, de notes historiques \& politiques; continuée jusqu'à présent, \& enrichie de tailles-douces [par H. Bourgeois du Chastenet et Vogel], 8 vol. in- $8^{\circ}$, Amsterdam 1733; IPM: t. VII, p. 780; IPO: ibid., p. 81-212; autre tirage, 2 vol. in $4^{\circ}$, ibid. 1733; IPM: t. II, p. 412-436; IPO: ibid., p. 437-480.

140 ID., The History of the Empire; containing its rise, growth, revolutions [...]. Done into English from the best edition and continued down to this time with divers remarks, and new authentic pieces [par H. Bourgeois du Chastenet], 2 vol., Londres 1727-1729.

141 ID., History of the Empire, from the time of Charlemagne to the present Emperor Charles VI. Being an appendix to the history of the Roman Empire, by the sieur Heiss. Done into English from the best edition; with divers remarks and new authentic pieces, by Paul Chamberlen, Londres 1730.

142 ID., The History of the German Empire [...] being a continuation of M. Echard's [sc. Lauwrence Echard (1670?-1730)] Roman History, Londres 1731.

${ }^{143}$ Le fait que cette partie ait été publiée dans un volume à part, sous le titre de »Divers traitez de paix «, a abouti à une certaine confusion dans les bibliothèques. En effet, bien que le titre précise que ces traités devaient servir de "preuves à l'Histoire de l'Empire«, les deux premiers tomes du livre de Heiss sont souvent rangés sous le nom de l'auteur, Heiss, tandis que le tome III figure dans le catalogue des anonymes sub voce »Divers traitez de paix «; c'est le cas à la BNF (voir ci-dessus).

144 Cf. Ant[oine]-Alex[andre] BARBIER, Dictionnaire des ouvrages anonymes, troisième édition, revue et augmentée par Olivier Barbier, René et Paul BiLlard, 4 vol., Paris 18721879, ici t. II, p. 683; J.-M. QUÉRARD, La France littéraire ou Dictionnaire bibliographique des savants, historiens et gens de lettres de la France, ainsi que des littérateurs étrangers qui ont écrit en français, plus particulièrement pendant les XVIII et XIX ${ }^{e}$ siècles, 12 vol. Paris 1827-1864 (reproduction en fac-similé, Mayenne 1964), ici t. I, p. 471. C'est la conjecture qui semble être généralement admise depuis; pour une attribution différente de Lenglet, voir le chapitre IX.

145 Selon Adelung, il serait mort avant 1720, cf. Johann Christoph ADELUNG, Fortsetzung und Ergänzungen zu Christian Gottlieb Jöchers allgemeinen Gelehrten-Lexico, worin die 
qu'il fut avocat au parlement de Paris. Il rédigea un certain nombre d'autres ouvrages d'histoire et se fit connaître comme traducteur et continuateur ${ }^{146}$. Les éditions de 1731 furent encore augmentées de notes et continuées jusqu'en 1724 par »V.G.J.D.G.S.«. Selon Quérard, il s'agit d'un initialisme qui est à déchiffrer »Vogel, grand-juge des gardes suisses «147. Même si Quérard ne précise pas son prénom, il est certain qu'il s'agit de Franz-Adam Vogel, issu d'une famille du canton de Schaffhouse citée dans ce canton depuis le milieu du XVe siècle ${ }^{148}$. Vogel, mort en 1749, était effectivement à l'époque grand juge des gardes suisses à Paris. On a de lui plusieurs ouvrages de jurisprudence; il est l'auteur entre autres écrits d'un »Traité historique et politique « sur les relations franco-suisses et sur les privilèges des Suisses en France ${ }^{149}$; il fit aussi une édition du code pénal de Charles Quint (la fameuse »Caroline $\ll)^{150}$, destinée à l'usage des conseils de guerre des troupes suisses ${ }^{151}$. Les éditions de 1733 sont encore enrichies d'un "Discours pré-

Schriftsteller aller Stände nach ihren vornehmsten Lebensumständen und Schriften beschrieben werden, t. II, Leipzig 1787, col. 267.

146 Cf. Nouvelle Biographie générale, t. 7, col. 82; [Louis Gabriel] MıCHAUd (dir.), Biographie universelle ancienne et moderne. Nouvelle édition, 45 vol., Paris 1843-[1865] (reproduction en fac-similié, Bad Feilnbach 1998), ici t. 5, p. 314.

147 J.-M. Quérard, Les supercheries littéraires dévoilées [.. .], 4 vol., Paris 1869-1870 (reproduction en fac-similé, Mayenne 1964), ici t. III, col. 936.

148 Cf. Dictionnaire historique et biographique de la Suisse publié avec la recommandation de la Société générale suisse d'histoire et sous la direction de Marcel GODET, Henri TüRLER, Victor ATTINGER, 7 vol. et supplément, Neuchâtel 1921-1934, ici t. VII, p. 161.

149 Sur son cuvre littéraire, cf. QUÉRARD, Supercheries, t. III, col. 936; ID., La France littéraire, t. X, p. 259.

150 Édition du texte original allemand: RADBRUCH (éd.), Die Peinliche Gerichtsordnung; édition du texte allemand avec de nombreux documents qui en éclairent l'origine: J[osef] KoHLER et al. (éd.), Die Carolina und ihre Vorgängerurkunden. Text, Erläuterung und Geschichte, 4 vol., Halle/Saale 1900-1915.

151 La première édition date de 1734: [Franz-Adam VoGEL], Code criminel de l'Empereur Charles V. vulgairement appellé la Caroline, Paris 1734. Vogel, grand juge des gardes suisses du roi, signe l'épître dédicatoire au baron de Besenval, colonel des gardes suisses; il dit que l'ouvrage a été entrepris et exécuté sur son ordre. Après la traduction française de la Caroline (p. 1-316), qui est accompagnée des observations très détaillées de l'auteur (plus longues que le texte de la Caroline même, ajoutées à la fin de chaque article), l'ouvrage contient d'»Autres matieres concernant la Justice Criminelle«, c'est-à-dire, surtout, les règlements concernant la discipline militaire (à partir de la p. 317). L'auteur indique, en marge du texte, les principaux auteurs allemands qui ont écrit sur le droit pénal. L'objectif de cette publication du Code pénal de Charles Quint et des observations de Vogel est évident: il est d'ordre pratique, puisque le texte doit servir à juger les membres des gardes suisses. Dans l'»Approbation« signée Courchetet, datée Paris 27 mars 1733 (imprimée à la fin du volume), ce livre est jugé „nécessaire pour les Jugemens Militaires que Messieurs les Officiers Suisses rendent dans le Royaume «. Vogel, rappelle, dans sa »Preface« (sans pagination) pourquoi les gardes suisses suivent les règles du Code criminel de Charles Quint; en effet, quand ce code fut promulgué, à la diète d'Augsbourg, en 1530, et à celle de Ratisbonne, en 1532, il ne s'agissait que d'une confirmation des lois qui avaient "été de tout tems en usage dans les Tribunaux de l'Allemagne. C'est de cette source que les a tirées la Suisse lorsqu'elle étoit autrefois un de ses membres. les plus considérables de ses Citez étant même anciennement décorées du titre de Villes Impériales«. À plusieurs reprises, cet ouvrage fut réédité au cours du XVIIle siècle en France et à l'étranger. 
liminaire « qui doit être attribué à Vogel ou aux éditeurs néerlandais ${ }^{152}$. Par rapport aux éditions enrichies, les exemplaires des traités de Westphalie ont subi quelques changements dans ces éditions augmentées. Tout d'abord, on y a ajouté des notes de bas de page. Celles-ci ne sont cependant que d'une valeur restreinte, offrant quelques notions sur un certain nombre de lieux, de personnes et d'événements. Il n'y a rien qui ressemble à une analyse ou à une explication plus approfondies des traités. Outre les changements intervenus suite à une nouvelle subdivision des articles, et sans prendre en considération les différences affectant les seuls noms propres, l'on constate quelque deux cent cinquante passages où les éditions de 1684 à 1733 font état de leçons différentes ${ }^{153}$. En somme, on peut faire le bilan suivant: le texte est toujours pris dans l'édition précédente; l'édition de 1711 se reporte toutefois à la première édition, en y apportant certains changements considérables. Il y a donc deux familles différentes: éditions du XVII et éditions du XVIII e siècle. Les deux éditions parues en une même année, respectivement en 1731 et en 1733 , sont très proches, mais il n'y a pas d'éditions identiques. Si les doublons sont le plus souvent corrigés, les lacunes augmentent, du fait qu'on se reporte généralement à l'impression précédente, d'édition en édition; avec une seule rupture, on l'a dit, en 1711. Certes, il s'agit le plus souvent de lacunes d'un seul mot, qui ne changent pas le sens du texte. Mais les petites différences peuvent parfois entraîner des conséquences considérables. Par exemple, l'empereur n'eût probablement pas signé la paix sans la clause arrêtant que les alliances conclues par les princes d'Empire ne devaient être dirigées »ni contre l'Empereur ni contre l'Empire«. Or, cette clause, l'une des plus fameuses de toute la paix, manque dans les éditions du traité de Münster de 1731 et, soit qu'on ne s'en aperçût pas, soit qu'on n'accordât pas trop d'importance à cette stipulation en France, où elle fut considérée souvent comme une clause insérée seulement pour la forme, l'ouvrage de Heiss réapparut à deux reprises, en 1733, sans qu'on suppléât à ce défaut.

Si la traduction de Heiss est devenue le point de référence de toute la postérité, c'est certainement dû à la grande qualité et à la large diffusion de son ouvrage. Mais il convient de souligner qu'une partie des éditions suivantes n'ont pas directement repris le texte de Heiss. En effet, son succès s'explique aussi par le fait que sa traduction est entrée dans d'autres ouvrages de grande qualité qui eux-aussi font autorité dans leur domaine, en partie jusqu'à nos jours. Comme la plupart d'entre eux sont bien connus, il suffira d'en rappeler ici quelques caractéristiques essentielles. Le premier à avoir réédité un des traités dans

152 Au sujet du jugement que les continuateurs de l'»Histoire de l'Empire « portent sur Heiss et de leurs opinions politiques, différentes des siennes, à plusieurs égards, cf. le chapitre IX. 153 Résultat de la collation de toutes les éditions que nous avons pu repérer et qui sont citées dans notre bibliographie (au moment de la publication de notre article BRAUN, Traductions, ici p. 138, nous avons indiqué le même chiffre pour le collationnement de huit éditions sur neuf, celle de 1711 ne nous étant pas accessible; or, comme cette dernière, que nous avons eu l'occasion de consulter entre-temps, correspond aux éditions postérieures, ce nombre ne change pas, même si l'on prend en considération toutes les éditions). 
la version de Heiss fut probablement Frédéric Léonard dans son recueil de 1693 que nous avons déjà évoqué. Il y inséra le traité d'Osnabrück de Heiss, tout en gardant sa propre traduction de celui de Münster. C'est une circonstance très importante. Car c'est dans le recueil de Léonard que vont puiser la plupart des compilateurs des décennies suivantes, et avant tout les éditeurs néerlandais. Ainsi, par l'intermédiaire de Léonard ${ }^{154}$, la paix d'Osnabrück d'après Heiss entre notamment dans le »Grand Recueil« de Hollande de 1700 et le »Corps universel diplomatique«, de Jean Dumont. C'est peut-être la médiocrité de la paix de Münster que Léonard offrait qui fit que ces deux recueils donnèrent le texte de ce dernier traité en latin.

La première desdites collections, le »Recueil des traitez de paix, de trêve, de neutralité, etc.«, est aujourd'hui le plus souvent cité »Bernard «, d'après son compilateur, ou "Moetjens «, d'après son éditeur; mais les contemporains, ce qui est significatif de son influence, l'appelait tout court le »Grand Recueil«155. Il s'agit en effet d'une entreprise de libraire. À l'instigation d'Adrian Moetjens, libraire à La Haye, deux puissants groupes d'éditeurs-libraires hollandais, l'un de La Haye, l'autre d'Amsterdam, se réunirent pour publier une nouvelle collection de traités qui surpasserait toutes les précédentes par l'abondance des documents. La tâche de l'établir fut confiée à un théologien protestant français qui s'était réfugié aux Pays-Bas, Jacques Bernard ${ }^{156}$. Les quatre volumes de cette collection in-folio parurent enfin en 1700 et comprirent plus de mille cinq cents documents allant de 536 av. J.-C. à la fin du XVIIe siècle ${ }^{157}$.

Le »Corps universel diplomatique «, de Jean Dumont ${ }^{158}$, était au départ conçu comme une réédition du »Grand Recueil«. Dumont, né à Rouen le 13 janvier 1667 et mort à Vienne en Autriche le 13 mai 1727, était protestant et dut quitter la France après avoir été incarcéré à Strasbourg pour ses prises de position religieuses et politiques ${ }^{159}$. Après avoir traversé l'Europe d'un bout à l'autre, il

154 La filiation Heiss-Léonard-»Grand Recueil«-»Corps universel« est à établir clairement à partir des leçons divergentes.

155 Recueil des traitez de paix [dit Grand Recueil de Hollande]. Pour l'édition du texte latin de la paix de Münster, l'éditeur (t. III, p. 528) signale ces éditions antérieures: "Theatrum Pacis Tom. I. pag. 206 Freder. Leonard. Tom. III. \&c. «; pour le texte français de la paix d'Osnabrück, ibid., p. 541: »Heis Histoire de l'Empire. Tom. III. pag. 54. Freder. Leonard. Tom. III.«. Cf. HeIss, Histoire de l'Empire (édition de 1685, t. III, p. 54-153, ou édition de 1694, t. III, p. 54-153) et LÉONARD, Recueil de tous les traités modernes, t. III (1693).

156 Jacques Bernard, né en 1658 à Nyons (Drôme), s'installa en Hollande en 1685; ce polygraphe obtint la chaire de philosophie à l'université de Leyde, en 1712; il mourut en 1718; P. HAMON, article »Bernard (Jacques)«, dans: Dictionnaire de biographie française, t. VI (1954), col. 59. Par ailleurs, il était le cousin de Jean Le Clerc; sur ce dernier: BRAUN, Le Clerc.

157 Cf. Toscano, History, p. 56-57; ID., Storia, p. 72-73; VAST, Les grands traités, p. XI et 8.

158 Dumont, Corps universel; IPO: t. VI (1728), première partie, p. 469-490.

159 Pour la vie et les æuvres de Dumont, cf. Biographie universelle, t. XII, p. 229-231; Nouvelle Biographie générale, t. 15, col. 197-198; CIORANESCU, Bibliographie de la littérature 
s'arrêta à La Haye en 1692 et se mit à donner des cours de droit public. C'est à cette époque qu'il commença à se consacrer aux relations internationales et en particulier à l'histoire des traités. Au début du XVIIIe siècle, il conçut le projet d'une réédition du »Grand Recueil« et se mit à rassembler des documents à cet effet. Bien qu'il fût conseiller et historiographe de l'empereur Charles VI à Vienne au moment où devait paraître ce nouveau »Grand Recueil «, il ne cessa de consacrer une grande partie de son temps à cet ouvrage. S'étant vu confier la charge de préparer la réédition du fameux recueil probablement en 1716, son obsession l'amena à rassembler en six ans quelque cinquante mille pièces, en hantant toutes les cours d'Europe pour qu'on lui communiquât les documents intéressants. Les éditeurs hollandais, effrayés de l'ampleur qu'avait pris le projet de Dumont, lui imposèrent des restrictions quant au nombre des pièces, et l'obligèrent à suivre le plan général du »Grand Recueil«. L'ouvrage qui fut finalement publié n'avait pourtant pas grand-chose à voir avec celui-ci. Il comprit en huit volumes in-folio plus de dix mille documents recouvrant la période de Charlemagne à 1730, et fut complété par un volume pour la période avant Charlemagne, établi par Jean Barbeyrac ${ }^{160}$, professeur à Groningue, ainsi que par deux suppléments en quatre volumes, compilés par Jean Rousset ${ }^{161}$. Ces suppléments furent publiés en 1739162. Le soin avec lequel on entreprit cette publication se manifeste entre autres dans la grande fidélité du texte au traité d'Osna-

française du dix-huitième siècle, t. I, p. 737-738; Y. DESTIANGES, article »Dumont (Jean)«, dans: Dictionnaire de biographie française, t. XII (1970), col. 220-221.

160 Le jurisconsulte calviniste Jean de Barbeyrac, né à Béziers, en 1674, quitta la France après la révocation de l'édit de Nantes, en 1686. Il étudia d'abord à Lausanne et à Genève, avant de s'installer à Francfort, puis à Berlin, où il s'occupa du droit et dispensa des cours d'histoire au collège français. Pourvu d'une chaire d'histoire du droit à l'académie de Lausanne, en 1710, puis à l'université de Groningen, de 1717 à 1744, année de sa mort, Barbeyrac était aussi associé à l'Académie des sciences de Berlin, depuis 1713. Il se fit connaître surtout par ses éditions et ses traductions libres, qui, au dire d'un biographe, »donnent lieu à des changements de plans, à des suppressions, à des commentaires, à d'innombrables notes" et représentent »à proprement parler d'ingénieux remaniements«. En particulier, il se fit remarquer comme traducteur de Pufendorf, en 1706 et 1707 ( $\$$ Le droit de la nature et des gens «, "Les devoirs de l'homme«) et de Grotius (»De jure belli ac pacis«, en 1724, suivant l'édition du texte latin publiée par Barbeyrac en 1720). Ses longs commentaires sur Samuel von Pufendorf furent même traduits en anglais; cf. Roman D' AMAT, article "Barbeyrac (Jean de)«, dans: Dictionnaire de biographie française, t. V (1951), col. 297-298; OTHMER, Berlin und die Verbreitung des Naturrechts.

161 Pour Jean Rousset de Missy (1686-1762), voir Archives biographiques françaises I 917, 214-228.

162 [Jean] BARBEYRAC, [Jean] ROUSSET [DE MISSY], Supplement au corps universel diplomatique du droit des gens, contenant un recueil des traitez [...]. [I.] Histoire des anciens traitez [...] depuis les temps les plus reculez jusques à l'Empereur Charlemagne, par Mr. Barbeyrac, 2 parties en 1 vol.; [II.-III.] I.-II. Supplement au corps universel diplomatique du droit des gens, contenant un recueil des traitez [...] Qui ont échapé aux prémieres recherches de Mr. Du Mont, continué jusqu'à présent par Mr. Rousset, 4 parties en 2 vol.; [IV.-V.] I.-II. Le cérémonial diplomatique [...], par Mr. Rousset. 2 vol., Amsterdam, La Haye 1739. Pour le "Corps universel« et ses suppléments, cf. VAST, p. XI-XII; TOSCANO, History, p. 59-64; ID., Storia, p. 76-84; RÉmY-Limousin, Traités internationaux, nos $\mathrm{R} 1$ et $\mathrm{R} 2$. 
brück par rapport à celui qu'en donne le »Grand Recueil«. En effet, il y avait, aux XVII ${ }^{e}$ et XVIII ${ }^{e}$ siècles, bon nombre de difficultés éditoriales qui font que de nombreuses éditions de l'époque sont assez infidèles à leurs devancières. Le "Corps universel diplomatique " fait exception ${ }^{163}$. C'était un ouvrage que les contemporains considéraient comme définitif. Mis à part quelques recueils nationaux ou axés sur des traités récents, l'âge d'or des collections de traités est clos avec Jean Dumont. La fin du XVIII siècle devait voir des travaux d'un nouveau type, universitaires, et qui sont liés aux noms des professeurs allemands Friedrich August Wilhelm Wenck et Georg Friedrich von Martens ${ }^{164}$. Outre le »Corps universel diplomatique«, deux autres petits recueils contiennent les traités de Westphalie. Ils sont aussi attribués à Dumont. Le premier parut à Luxembourg et connut probablement trois éditions ${ }^{165}$. C'est une édition de la paix de Ryswick à laquelle on a ajouté des traités antérieurs à 1697166. Chaque traité a un frontispice et une pagination particuliers ${ }^{167}$. La deuxième édition, enrichie ${ }^{168}$, date de 1698 , et la troisième édition de 1703 ; nous n'avons pu retrouver d'exemplaire de la première édition ${ }^{169}$. Mais à en juger par le frontispice, qui qualifie l'édition de 1698 d'augmentée, et l'»Avis« du libraire, il y avait bien une édition antérieure, qui comprenait, très certainement, les traités de paix depuis le traité de Münster jusqu'aux traités de Ryswick, mais non celui d'Osnabrück; ce dernier compléta l'édition de 1698 170. Étant donné qu'elle devait contenir les traités de Ryswick conclus entre le 20 et le 30 octobre 1697 , cette édition ne peut dater que de l'extrême fin de 1697 ou, comme la deuxième édi-

163 Cf. sa traduction: Dumont, Corps universel, t. VI (1728), première partie, p. 429-441. Une seule lacune de plusieurs mots à la fin de l'art. V,24 IPO (art. V \& 8 selon la division du "Corps universel«, ici p. 475). À plusieurs endroits, Dumont corrige même des fautes du »Grand Recueil« de 1700.

164 Cf. RémY-Limousin, Traités internationaux, nos $R 52$ et $R 53$.

165 ANDRÉ, Les sources de l'histoire de France, t. V, p. 5 notice 3209, n'en connaît qu'une seule édition, datant de 1698 .

166 [Jean Dumont], Recueil des principaux traitez de paix, faits et conclus pendant ce siecle [...], Luxembourg 1698; cf. RÉMY-LIMOUSIN, Traités internationaux, $\mathrm{n}^{\circ} \mathrm{R} 43$ (qui transcrit incorrectement l'ex-dono; voir notre bibliographie). De Jean Dumont selon une notice de la Library of Congress, Washington, qui indique une édition de 1703 (cote: JX132.D77 1703); elle porterait le même titre que la précédente, mais au lieu de l'expression "pendant ce siecle«, on y lirait: »pendant le dix-septième siecle« (pagination particulière pour chaque traité), cf. National Union Catalog (voir chapitre IX, n. 134), t. 151 (1971), p. 474.

167 Les traités de Münster et d'Osnabrück sont les deux premiers traités dans l'édition de 1698.

168 D'après le frontispice, le recueil a été »Augmenté dans cette édition de trois traitez, \& de l'article separé touchant le Roy d'Angleterre Guillaume III. que l'on a marqué d'une étoile pour les distinguer «; entre les pièces marquées d'une étoile, on note le traité d'Osnabrück qui, par conséquent, n'a certainement pas été publié dans la première édition.

169 Cette numérotation des éditions n'est pas authentique; nous n'avons vu que l'exemplaire de la BNF datant de 1698. Selon Braun, OschmanN, RePGEN, APW III B 1/2, p. 173, n. 12 , l'édition de 1703 se trouve, outre aux États-Unis, peut-être aussi à la bibliothèque de la ville de Trèves (cote: X I $158^{\circ}$ ); toutefois, Oschmann n'a pas pu consulter cet exemplaire.

170 En effet, marqué d'une étoile, contrairement aux traités de Münster et de Ryswick, il n'a très certainement pas fait partie de l'édition princeps. 
tion, de 1698. Le bref délai dans lequel elle fut épuisée s'expliquerait par la conclusion de la paix de Ryswick, qui avait certainement accru l'intérêt accordé aux affaires internationales ${ }^{171}$. Ce recueil, publié au Luxembourg, reprend la traduction du traité de Münster ${ }^{172}$ de Heiss et, plus précisément, il s'agit probablement de celle qui fut publiée dans la troisième édition de l'»Histoire de l'Empire «173. En revanche, pour le traité d'Osnabrück, il ne reproduit que le résumé de l'accommodement du 6 août 1648, publié dans la »Gazette«, les 25 novembre et 2 décembre $1648^{174}$. Cette traduction du traité de Münster est d'ailleurs différente de celle qui figure dans un autre recueil qui date également de 1698 et fut publié par les imprimeurs associés à Paris ${ }^{175}$. Il semble qu'au Luxembourg la paix d'Utrecht ait entraîné l'édition d'un recueil de traités en 1714, sans que nous sachions exactement quels documents il contenait ${ }^{176}$. Un $»$ Recueil de divers traitez de paix «, généralement attribué à Jean Dumont, parut enfin en 1707 chez Adrian Moetjens, à La Haye ${ }^{177}$. Il contient une traduction du traité de Münster qui provient probablement de la deuxième édition de Heiss ${ }^{178}$. Parmi les recueils de traités, il faut encore noter un »Recueil de tous les traités de paix faits et conclus pendant ce siecle entre les potentats de l'Europe«, publié à Paris, par les »Imprimeurs associés«, peu après la signature de la paix de Rys-

171 En tout cas, c'est la conclusion de la paix de Ryswick qui a donné lieu à l'édition de ce recueil; en effet, dans l'»Avis « du libraire, qui se trouve au verso du frontispice, l'éditeur explique qu'ayant mis les traités de Ryswick sous presse il s'est aperçu de la nécessité de connaître les principaux traités depuis la paix de Westphalie pour les comprendre.

172 [Dumonr], Recueil des principaux traitez de paix, [document 1:] Traité de paix signé à Munster en Westphalie le 24. octobre 1648. par les ambassadeurs plénipotentiaires de leurs Majestez Imperiale \& Trés-Chrétienne, \& par les autres députez plénipotentiaires des électeurs, princes \& états du saint Empire Romain, Luxembourg 1698 (p. [1]-96).

173 Ceci résulte du collationnement des éditions. Il convient toutefois de souligner que les différences entre les deuxième et troisième éditions de HEIss, Histoire de l'Empire (1685 et 1694) sont extrêmement rares, de sorte qu'on ne peut pas tout à fait exclure la possibilité qu'on ait pris le texte dans la seconde édition, même si cela paraît moins probable. En revanche, toute autre filiation est exclue (à moins qu'il n'y ait quelque impression intermédiaire qui nous ait échappé).

174 [Dumont], Recueil des principaux traitez de paix, [document 2:] Abregé du traité et des articles de paix, dressez à Osnabruk entre les plenipotentiaires de l'Empereur \& de la Reine \& couronne de Suede, en presence des états de l'Empire, Luxembourg 1698 (p. [1]-102); c'est le texte du 6 août 1648, avec les deux articles suspensifs.

175 Voir ci-dessous.

176 Cf. Braun, OschmanN, RePGeN, APW III B 1/2, p. 173, n. 12, où Oschmann indique un »Recueil des principaux Traitez faits \& conclus depuis le Traité de Westphalie en 1648. jusqu'au traité d'Utrecht en 1714. IV. Volumes in 12. Luxembourg 1708-1714«; cependant, elle a tiré cette notice d'une bibliographie du XVIII ${ }^{e}$ siècle et n'a pas pu la vérifier, faute d'en retrouver un exemplaire.

177 [Jean DumonT], Recueil de divers traitez de paix, de confederation, d'alliance, de commerce, \&c. faits depuis soixante ans, entre les Etats souverains de l'Europe [...], La Haye 1707; cf. RémY-LimOuSIN, Traités internationaux, $n^{\circ} \mathrm{R} 34$. Traité de Münster: première partie, p. 33-93.

178 Rappelons que que les différences entre les deuxième et troisième éditions de HEISs, Histoire de l'Empire (1685 et 1694) sont très rares. 
wick, en 1698; ce recueil comprend une édition du traité de Münster, en français ${ }^{179}$. C'est donc encore une fois l'actualité politique qui a fourni l'occasion de publier une collection de traités ${ }^{180}$. Les traités qui composent ce recueil ont des frontispices particuliers; la reproduction des armes de France aux différents frontispices accorde un caractère officieux à cette édition, mais le frontispice commun à tous les traités, placé en tête du recueil, ne comporte pas cette distinction. Fait unique: le traité de Münster est divisé en cent vingt-cinq paragraphes, une subdivision qui n'a pu être retrouvée dans aucune autre édition française, latine ou allemande ${ }^{181}$. Cependant, le texte du traité ne représente pas une nouvelle traduction: il a été puisé dans l'édition de Léonard; en règle générale, même les erreurs évidentes n'ont pas été corrigées. La seule nouveauté est donc la numérotation des paragraphes ${ }^{182}$.

Un autre ouvrage reprenant la traduction de Heiss et faisant autorité jusqu'à nos jours est l'»Histoire du traité de Westphalie« du père Bougeant. Bougeant, né à Quimper le 4 novembre 1690 et mort à Paris le 7 janvier $1743^{183}$, était jésuite et professeur au collège Louis-le-Grand à Paris; polygraphe qui écrivait sur pratiquement tous les arts et sciences de son temps, il représente le type même de l'érudit universel. D'abord connu pour ses raisonnements philosophiques et comme écrivain frivole, il ne se fit une belle réputation d'historien que par son »Histoire du traité de Westphalie«, parue à titre posthume en 1744, mais précédée d'une "Histoire des guerres et des negociations qui precederent le traité de Vestphalie « en $1727^{184}$, histoire que Bougeant avait écrite à l'instigation de

179 Recueil de tous les traités de paix faits et conclus pendant ce siecle entre les potentats de l'Europe, Paris 1698; le texte de l'IPM se trouve p. 5-65; chaque traité a un frontispice particulier, celui de l'IPM porte le titre suivant: Traité de paix entre l'Empereur, la France, et les électeurs, princes, et états du Saint Empire, conclu à Munster en Westphalie le vingt-quatre octobre 1648, avec l'année de publication (1698) et les armes de France (trois fleurs de lys, timbrées de la couronne royale), mais sans indication du lieu de publication.

180 Rappelons qu'en 1698 un autre recueil de traités, attribué à Jean Dumont, parut au Luxembourg; [DUMONT], Recueil des principaux traitez de paix (voir ci-dessus).

181 Pour le problème de la numérotation des articles et des paragraphes des traités de Westphalie, cf. les explications très précises et exhaustives d'Antje OSCHMANN, chapitre »Initien und Gliederungen des IPO und des IPM $\ll$, dans: BRAUN, OSCHMANN, REPGEN, APW III B $1 /$ 2, p. $685-710$.

182 Les paragraphes sont d'ailleurs les mêmes que dans LÉONARD, Traité de paix (1679), sauf que, chez Léonard, ces paragraphes n'étaient pas numérotés.

183 Et non en 1749, comme le dit Cioranescu. Pour Bougeant, cf. Archives biographiques françaises I 134, 391-410; II 13, 176; M. PREvOST, article »Bougeant (Guillaume-Hyacinthe)«, dans: Dictionnaire de biographie française, t. VI (1954), col. 1290; cf. aussi sur la vie de Bougeant, Biographie universelle, t. V, p. 299-300; Nouvelle Biographie générale, t. 6, col. 907; sur ses cuvres et les travaux qu'on lui a consacrés, Carlos SOMMERvoGEL. (S.J.), Bibliothèque de la Compagnie de Jésus. Bibliographie et histoire. Nouvelle édition publiée par la Province de Belgique, 12 vol., Bruxelles, Paris, Toulouse ${ }^{3} 1890-1932$ (reproduction en fac-similé, Louvain, 1960), ici t. I col. 1873-1886; CIORANESCU, Bibliographie de la littérature française du dix-huitième siècle, t. I, p. 377-379.

184 [Guillaume-Hyacinthe] BOUGEANT (S.J.), Histoire des guerres et des negociations qui precederent le traité de Vestphalie [...], 1 vol. in- $4^{\circ}$ ou 2 vol. in-12, Paris 1727; ID., Histoire 
l'héritier (ou du propriétaire) des papiers du comte d'Avaux ${ }^{185}$. Ces deux ouvrages n'en forment à vrai dire qu'un seul ${ }^{186}$. Celui-ci fut réimprimé sous des titres divergents en 1751 et $1767^{187}$. L'»Histoire" de Bougeant se fonde surtout sur les mémoires du comte d'Avaux. Elle n'est évidemment à utiliser qu'avec quelques précautions par les historiens de nos jours, mais étant resté le seul véritable ouvrage français de référence sur la paix de Westphalie, elle constitue indéniablement une »source à laquelle on a toujours puisé «188. Étant donné que Bougeant - du moins si l'on en croit ses propres assertions - avait à sa disposition les papiers d'Avaux, qui ne sont pas intégralement parvenus jusqu'à nos jours, son ouvrage restera toujours »indispensable «, comme le remarque très justement Tischer ${ }^{189}$.

La traduction du traité d'Osnabrück de Heiss fut encore publiée dans "Le droit public germanique « d'Éléazar de Mauvillon ${ }^{190}$. Si l'on peut constater que le droit

du traité de Westphalie, ou des negociations qui se firent à Munster \& à Osnabrug [...], 2 vol. (ou 3 vol. avec l'ouvrage précédent) in- $4^{\circ}$, Paris 1744; autres éditions des deux ouvrages en 1744 (6 vol. in-12), 1751 (6 vol. in-12) et 1767 (3 vol. in-40). Le texte des traités de Westphalie se trouve dans l'édition de 1744 (in-40): IPM: t. II, p. 507-548; IPO: ibid., p. 549631; tirage in-12: IPM: t. VI, p. 215-280; IPO: ibid., p. 281-412 (mêmes paginations dans les rééditions de l'ouvrage intégral, $t$. III in- $4^{\circ}$ ou $t$. VI in-12).

185 Il l'explique dans la préface de l'édition de 1727 ; $\mathrm{cf}$. l'édition in $-4^{\circ}$, six pages non numérotées, placées entre la dédicace et le privilège royal, ici fol. ã iij. Dans cette préface, Bougeant explique aussi que le présent volume ne doit servir qu'à comprendre mieux les négociations du congrès de Westphalie, qui feront l'objet du ou des volumes suivants.

186 Comme on peut le voir par les approbations du garde des sceaux et du provincial de la Compagnie de Jésus, datées respectivement du 15 juin et du 16 septembre 1726, ainsi que par le privilège du roi du 4 juillet de la même année (cédé par Bougeant à l'imprimeur Jean Mariette le 14 et enregistré le 17 septembre), l'Histoire de la guerre de Trente Ans avait été terminée en été 1726. Selon le plan originel, Bougeant avait l'intention de publier les mémoires du comte d'Avaux, d'abord en même temps que l'Histoire de la guerre de Trente Ans, puis à la fin du dernier volume de l'Histoire du traité de Westphalie, mais ces projets ne furent pas exécutés; toutefois, il les expose à la fin de la préface de son Histoire de la guerre de Trente Ans; cf. ibid., ici fol. [ě'].

187 En 1751 sous le titre d'»Histoire du traité de Westphalie« et en 1767 sous le titre d'»Histoire des guerres et des negociations«.

188 Consécration s'il en est pour un auteur catholique français, il fut traduit en allemand par un théologien protestant, Friedrich Eberhard Rambach (né à Pfullendorf en 1708, mort à Breslau en 1775); voir Wilhelm Hyacinth BougEANT, Historie des dreyBigjährigen Krieges und des darauf erfolgten Westphälischen Friedens. Aus dem Französischen übersetzt. Mit Anmerkungen und einer Vorrede begleitet von Friedrich Eberhard Rambach, 4 vol., Halle $1758-1760$.

189 Cf. TISCHER, Französische Diplomatie, p. 1-2: »Unentbehrlich bleibt Bougeants Werk schon deshalb, weil er nach eigenen Aussagen Papiere d'Avaux' zur Verfügung und also Material benutzte, das nicht mehr in vollem Umfang vorliegt«. Or, le fait que Bougeant n'ait pas disposé de la correspondance des autres négociateurs a aussi pu l'induire en erreur, voir ibid., p. 333. Cf. aussi l'article »Bougeant, Guillaume-Hyacinthe«, dans: CROXTON, TISCHER, The Peace of Westphalia, p. 33.

190 [Eléazar de MaUviLlon], Le Droit public germanique. Où l'on voit l'état présent de l'Empire, ses principales loix \& constitutions [...], 2 vol., Amsterdam 1749; 2 vol., Amsterdam, Leipzig 1756; édition de 1749, IPO: t. II, p. 312-460; édition de 1756: même pagina- 
public allemand était propagé en France principalement par des juristes alsaciens ou allemands passés au service du roi, Éléazar de Mauvillon est un contre-exemple. Né à Tarascon en Provence le 15 juillet 1712, il émigra jeune en Allemagne, peut-être à cause des difficultés que lui causait la profession de la religion réformée. Il se fit connaître comme traducteur, historien ${ }^{191}$ et grammairien et fut pendant quelque temps secrétaire privé de Frédéric-Auguste, électeur de Saxe et roi de Pologne ${ }^{192}$. C'est en 1749 qu'il publia son »Droit public germanique«, ouvrage très favorable aux électeurs, qu'il présentait comme des égaux de l'empereur. Il est intéressant de noter le choix des documents publiés en appendice et pris pour les lois fondamentales les plus importantes du Saint-Empire; car ce sont exactement les mêmes qui seront choisies cinq ans plus tard dans un autre ouvrage sur le droit public allemand, celui de Scheid ${ }^{193}$ : la Bulle d'or, la dernière capitulation impériale (celle de François Ier) et le traité d'Osnabrüick ${ }^{194}$. De plus, ce sont surtout ces trois documents - la capitulation changeant selon l'empereur en place - qui sont reçus par les juristes de langue française, parmi les lois constitutionnelles de l'Empire.

Une nouvelle traduction du traité d'Osnabrück fut publiée dans le »Traité sistématique touchant la connoissance de l'état du Saint Empire Romain de la nation allemande «, paru en quatre volumes entre 1751 et 1754 à Hanovre, en Allemagne. Barbier l'attribue à un certain »Scheid «, sans préciser de prénom; le catalogue de la Bibliothèque nationale suit celui du British Museum en citant comme auteur Chrétien-Louis Scheidt, historien allemand né le 26 septembre 1709 à Waldenbourg dans le pays de Hohenlohe et mort à Hanovre le 25 octobre $1761^{195}$. C'est

tion, même matériel typographique et mêmes fautes d'impression que dans l'édition précédente.

191 Outre ses publications sur les particularités des langues française et allemande, Mauvillon consacra notamment des biographies à de grands personnages politiques des XVIIe et XVIII ${ }^{e}$ siècles et rédigea un certain nombre d'ouvrages sur l'histoire militaire. L'un de ses plus grands succès de librairie fut son »Prince Eugène«, d'abord paru en 1740, qui vit au moins trois rééditions.

192 Mauvillon mourut en mai 1779, à Leipzig selon Cioranescu, à Brunswick d'après la "Biographie universelle« et la »Nouvelle Biographie générale«; cf. Biographie universelle, t. XXVII, p. 578-579; Nouvelle Biographie générale, t. 34, col. 450-451; Archives biographiques françaises I 724, 1-14; sur ses œuvres: CIORANESCU, Bibliographie de la littérature française du dix-huitième siècle, t. II, p. 1225.

193 Voir ci-dessous.

194 Le premier document en version bilingue (latin et français), les deux autres en français seulement. Pour la capitulation impériale et la Bulle d'or, cf. [MAUvillon], Le Droit public germanique (1749), t. II, p. [1]-311; (1756), t. II, p. 1-311.

195 Il n'est pas certain que Barbier ait pensé au même personnage, car il y avait bien d'autres savants qui portaient le même nom au XVIII ${ }^{e}$ siècle, qui s'écrivait, comme chez Barbier, »Scheid«, sans » $\ll$. Toutefois, à l'heure actuelle, le catalogue »Opale plus« de la BNF attribue toujours cet ouvrage à Chrétien-Louis (Christian-Ludwig) Scheidt, sans préciser les raisons de cette attribution. Cf. aussi Catalogue général des livres imprimés de la Bibliothèque nationale, publié par le ministère de l'Instruction publique et des Beaux-Arts (puis ministère de l'Éducation nationale). Auteurs, 231 vol. Paris 1897-1981, t. 164 (1943, col. 1141-1143, ici col. 1143; BARBIER, Dictionnaire des ouvrages anonymes, t. IV, col. 810. 
un personnage passionnant et en même temps tragique, qui mériterait bien qu'on lui accordât une biographie. Après avoir occupé une chaire de droit public à Copenhague, il s'installa finalement à Hanovre en 1748, en tant qu'historiographe et bibliothécaire royal. »Il fournit «, selon le jugement de la »Biographie universelle«, »une suite de travaux d'érudition qui auraient pu occuper dix savants «196. Mais ce personnage n'est pas l'auteur du »Traité sistématique«. En effet, à travers les comptes rendus publiés au XVIIIe siècle, nous pouvons prouver que celui-ci fut rédigé par son frère moins bien connu, Jean-Frédéric Scheid, lui aussi juriste et spécialiste du droit public ${ }^{197}$. Nous avons vu que Jean-Frédéric Scheid avait critiqué l'absence d'une édition fidèle des traités de Westphalie en France ${ }^{198}$, avant de publier lui-même, en 1754 , une traduction française de la paix d'Osnabrück ${ }^{199}$ d'après une copie latine collationnée sur l'original de Stockholm et publiée par Johann Gottfried von Meiern ${ }^{200}$. Ce fut en effet des archives du royaume de Suède que Meiern avait tiré une copie authentique du traité d'Osnabrück, conservée dans la bibliothèque royale de Hanovre ${ }^{201}$. Se fondant sur un texte latin rela-

196 Biographie universelle, t. XLI, p. 97-99, citation p. 98; Nouvelle Biographie générale, t. 43, col. 498-499; Deutsches Biographisches Archiv, troisième édition cumulée et augmentée [microfiches, parties I, éd. par Bernhard FABIAN sous la direction de Willi GORZNY, Munich et al. 1982, II et III, avec un index cumulé imprimé, en 8 vol.] éd. par Victor Herrero Mediavilla, Munich 2004, ici I 1093, 323-445; II 1136, 403-404; III 790, 255-256; 1050 , 209-211.

197 Étant donné qu'il s'agit d'un personnage mal connu, il n'est pas surprenant que son frère l'ait éclipsé; mais au XVIIre siècle, on savait déjà que Jean-Frédéric (Johann-Friedrich) Scheid était l'auteur du »Traité sistématique«, non pas son célèbre frère Chrétien-Louis Scheidt (ou Scheid), comme on l'a souvent supposé depuis la fin de l'Ancien Régime et comme nous l'avons autrefois suggéré nous-même, cf. BRAUN, Traductions, p. 140; cf. aussi Roger DufraisSE, Das Reich aus der Sicht der Encyclopédie méthodique 1784-1788, dans: Rainer A. MÜLLER (dir.), Bilder des Reiches. Tagung in Kooperation mit der schwäbischen Forschungsgemeinschaft und der Professur für Geschichte der Frühen Neuzeit der Katholischen Universität Eichstätt im Schwäbischen Bildungszentrum Kloster Irsee vom 20. März bis 23. März 1994, Sigmaringen 1997 (Irseer Schriften, 4), p. 123-153, ici p. 123. Pour l'attribution de cet ouvrage à Jean-Frédéric Scheid et pour sa biographie, voir aussi le chapitre $\mathrm{X}$; sur la base de nos recherches, la bonne indication de l'auteur se trouve aussi dans OschmanN, Meiern, p. 785, n. 28. Le nombre d'astérisques par lesquels Scheid remplace son nom, dans la dédicace du premier tome de son traité anonyme (SCHEID, Traité sistématique, p. ${ }^{*} 2-\left[{ }^{*} 3^{\prime}\right]$, ici p. [*3']), laisse d'ailleurs supposer qu'il ait préféré l'orthographe »Scheid « à »Scheidt«; l'exemplaire M.14702 à M.14705 de la BNF porte d'ailleurs le titre suivant sur le dos des volumes: „Droit Pub: Du S: Empire Par Scheid «; cf. aussi la mention manuscrite, t. I (M.14702) page non numérotée avant le frontispice: »Par Scheid«.

198 Voir l'introduction.

199 SCHEID, Traité sistématique, t. IV, p. 149-299.

200 Cf. BRaun, Traductions, p. 140; OsChMann, Meiern, p. 785-786.

201 Si on attribuait le »Traité sistématique« à Chrétien-Louis Scheidt, bibliothécaire à Hanovre, on pourrait supposer que celui-ci avait consulté personnellement la copie authentique, or la remarque que l'auteur du »Traité sistématique« fait sur cette copie est ambiguë, cf. BRAUN, Traductions, p. 140. En revanche, aucun lien personnel n'est attesté entre Jean-Frédéric Scheid et la bibliothèque de Hanovre, si ce n'est que son frère y fut bibliothécaire à partir de 1748: il est donc probable que Jean-Frédéric collationna sa traduction sur le texte latin 
tivement fidèle ${ }^{202}$, Scheid fournit un travail d'une précision et d'une rigueur méthodologiques que personne avant lui n' avait encore entrepris. Du travail exactissime de Scheid résulte donc la plus fidèle et la plus achevée traduction française du traité d'Osnabrück. L'on y trouvera aussi bon nombre de pièces relatives au traité, comme les ratifications et l'article secret ${ }^{203}$. Scheid a relevé pratiquement toutes les lacunes de Heiss ${ }^{204}$.

Il paraît utile de rappeler et de compléter certains résultats de ce chapitre concernant trois problèmes cruciaux: destinataires, diffusion et évolution des éditions de 1648 à 1754. Une grande partie des éditions de la »Gazette« jusqu'à Léonard poursuivent clairement des buts politiques et propagandistes, en essayant de justifier la politique gouvernementale et royale. Leur publication est

publié par Meiern; toutefois, il serait aussi possible qu'il ait sollicité l'aide de son frère pour faire collationner ce document sur la copie manuscrite authentique, mais aucun élément ne confirmant cette hypothèse, on doit s'en tenir à la solution la plus simple, c'est-à-dire la consultation de l'édition de Meiern par Jean-Frédéric Scheid, cf. ScHEID, Traité sistématique, t. IV, p. IV; pour les imprimés latins des traités de Westphalie et sur sa présentation de la version française, voir aussi ibid., p. V. En parlant du t. VII des "Acta Pacis « de Meiern, Scheid se trompe; en effet, les »Acta Pacis« comprennent seulement six volumes, publiés de 1734 à 1736; les traités de paix d'Osnabrück et de Münster furent publiés en 1738 dans un ouvrage ne faisant pas partie de la collection des »Acta Pacis«. Cf. MEIERN, Acta Pacis Westphalicae Publica; ID., Instrvmenta pacis caesareo-svecicvm et caesareo-gallicvm ex avthentico qvod in sanctioribvs regni svecici tabvlariis adservatvr in vsvm academicarvm accvratissime expressa et recognita de priorvm editionvm parvm accvratiorvm mendis et corrvptelis praefatvs est, Göttingen 1738. Sur Meiern, voir Guido BrauN, article »Meiern (or Meyern), Johann Gottfried von«, dans: CroxTON, TISCHER, The Peace of Westphalia, p. 189-190 (avec des références bibliographiques). Quelques erreurs dans l'édition du traité d'Osnabrück par Meiern, qui sont dues en partie aux fautes de lecture commises par les archivistes suédois en 1737 et en partie à Meiern lui-même, sont répertoriées par OschmanN, APW III B 1/1, p. CV-CVI.

202 En effet, si Scheid critiquait les versions françaises antérieures à la sienne, il savait bien que, même en Allemagne, il était difficile de trouver une bonne édition latine des traités, comme Meiern l'admettait lui-même; cf. ScHEID, Traité sistématique, t. IV, p. V. Au sujet des problèmes rencontrés pour trouver de bonnes éditions latines des traités, au XVII et encore dans une bonne partie du XVIIle siècle, cf. DickmanN, Der Westfälische Frieden, p. 500501. Or, on sait aujourd'hui que même le texte de Meiern n'était pas sans défauts, bien que son édition du traité d'Osnabrück, publiée en 1738, soit restée longtemps le modèle de référence; en ce qui concerne le traité de Münster, ce ne fut qu'en 1786 qu'une édition du texte latin, fondée sur un instrument original, fut publiée par Carl Friedrich Gerstlacher: ce fut le célèbre juriste Chrétien-Frédéric Pfeffel - dont nous avons déjà parlé dans l'introduction en personne qui, le 24 novembre 1785, collationna l'exemplaire du traité conservé à Versailles, dont Gerstlacher se servit l'année suivante (il s'agissait de l'original datant de mars 1649). La première édition critique tenant compte de tous les originaux des traités de Westphalie ne fut publiée qu'en 1998: OschmanN, APW III B 1/1; sur Meiern, Gerstlacher et les autres éditeurs des traités de Westphalie, voir ibid., p. XCVI-IC.

203 Cet article secret est publié en latin par OSCHMANN, APW III B 1/1, $\mathrm{n}^{\circ} 25$; pour cet article, cf. ibid., p. CVI-CVII.

204 Citons pourtant deux lacunes évidentes de Scheid: $1^{\circ} \mathrm{l}^{\prime}$ omission du plénipotentiaire autrichien Volmar dans le préambule; $2^{\circ}$ dans l'article VI, il faut lire »quasi-possession [d'une] pleine liberté« au lieu de »quasi-possession, pleine liberté«, cf. SCHEID, Traité sistématique, t. IV, p. [151]-155 et 226-227, en particulier p. 154 et 226. 
entreprise dans une intention politique bien précise, soit pour affronter des difficultés de politique intérieure (Fronde), soit pour accompagner une politique étrangère active et suggérer les points de vue français aux princes d'Europe (chambres de réunion), soit pour légitimer $a$ posteriori une prise de position politique ayant suscité la critique de certains milieux, soit enfin pour contribuer à la fabrication d'une certaine image de Louis XIV (chez Pierre Louvet) ${ }^{205}$. Le recueil de Léonard de 1693 s'adresse explicitement à deux catégories de personnes: d'un côté, aux souverains d'Europe et à leurs ministres, de l'autre "à ceux qui veulent savoir la vérité des points essentiels de l'Histoire «206, c'est-à-dire au public cultivé, qui avait pris goût à cette science, comme l'a montré Henri-Jean Martin ${ }^{207}$, et était obligé de savoir >parler histoire< en société. Cette collection poursuit donc un but double: contribuer à la gloire du roi en France même et à l'étranger, et servir d'instrument de travail aux politiques. Ce dernier objectif est celui des recueils parus auparavant aux Pays-Bas. Or, après Léonard, la publication des traités s'inspire certes encore souvent des événements sur l'échiquier international, comme le recueil du Luxembourg de la conclusion des traités de Ryswick, mais ces ouvrages n'ont plus de but politique tendancieux. Ils visent les compilations aussi bien que les livres d'histoire ou de jurisprudence - un public triple: le public instruit en général; le monde des savants, employés aux universités ou comme conseillers à la cour; et surtout le monde des diplomates. Cela résulte clairement des ouvrages et de l'analyse de leurs préfaces. Le fait que certains d'entre eux se disent par-dessus tout rédigés pour les futurs diplomates mérite notre attention particulière. Ce phénomène s'inscrit dans l'histoire générale de la diplomatie, qu'on commença, du moins théoriquement, à percevoir comme une véritable profession qui nécessitait une certaine formation. Bien qu'il n'y eût pas encore d'apprentissage régi par un »curriculum « pour les futurs négociateurs et ambassadeurs, ces ouvrages, destinés à fournir un tableau général de l'histoire, des traités et du droit des États européens, servaient en quelque sorte de premiers >livres scolaires ‘ à leur usage, qui venaient compléter les manuels de diplomates qu'on connaissait déjà depuis un certain temps. La "Bibliothèque politique« de La Maillardière se voulait explicitement »cours d'instruction « pour les négociateurs ${ }^{208}$. Ces livres traduisent par là l'institutionnalisation de la formation des diplomates, tout comme l'académie créée à cet effet en 1712 à l'instigation de Torcy et dirigée par Saint-Prest.

À la question des destinataires est liée celle de la diffusion de ces recueils. Or, pour la plupart des livres imprimés à l'époque moderne, on ne dispose d'aucune

205 Sur les »stratégies de la gloire« de Louis XIV, voir toujours la monographie de Peter BURKE, Louis XIV. Les stratégies de la gloire, Paris 1995 (traduit de l'anglais).

206 Cf. LÉONARD, Recueil des traitez de paix (1693), ici la »Lettre dédicatoire« au roi.

207 Cf. MARTIN, Livre, pouvoirs et société, passim.

208 La MaILlardière, Abregé dès principaux traités, p. XXVIII. La connaissance de la Constitution, de l'histoire et notamment des traités des pays constitue, pour cet auteur, l'essentiel du savoir du diplomate, puisqu'il déclare formellement: "toute autre connaissance est secondaire (ibid., p. XI). 
donnée chiffrée concernant le tirage, les livres de comptes de libraires faisant largement défaut. Toscano souligne cependant qu'un tirage de deux cent cinquante copies est très bas ${ }^{209}$; en revanche, selon Martin, les tirages de plus de trois mille exemplaires sont extrêmement rares. Si l'on peut effectivement faire quelques conjectures à ce sujet en établissant le seuil de rentabilité d'un ouvrage ${ }^{210}$, cela paraît une entreprise hasardeuse, car ce seuil dépend de paramètres multiples concernant les frais proportionnels (prix de fabrication d'un volume), la demande, et les frais fixes (composition du texte, mise en page), qui sont tous fort difficiles à évaluer. Pour notre propos, il suffit d'ailleurs de suivre les indications générales fournies par Martin pour donner une idée approximative de la diffusion des traités de Westphalie en langue française. Martin estime en effet que les ouvrages d'érudition ou les histoires générales ont été tirés, le plus souvent, à mille exemplaires ou un peu moins ${ }^{211}$. Pour rester prudent, on devra donc en moyenne partir d'un tirage légèrement inférieur à mille, peut-être autour de sept cents à huit cents exemplaires, en admettant que ce chiffre peut varier d'une édition à l'autre. Par ailleurs, le nombre de lecteurs par exemplaire est aussi très contesté, d'autant plus qu'à cause du prix très élevé des livres certains libraires les louaient, tout comme la »Gazette « ${ }^{212}$. Étant donné ces incertitudes, on n'avancera pas ici d'estimation chiffrée sur le nombre possible de lecteurs. À cause de la quantité des éditions des traités de Westphalie parues aux XVIIe et XVIII ${ }^{e}$ siècles, il est pourtant bien attesté qu'une élite cultivée, d'un nombre très restreint par rapport à la population totale, certes, mais qui dépassait le cercle des personnes employées dans des fonctions nécessitant de telles connaissances, a bien été au courant de ce dont il était question dans les traités conclus par le roi de France. Car le nombre de personnes qui avaient besoin de connaitre les traités à cause de leur fonction devait se compter par douzaines, les éditions au moins par centaines. Ce résultat s'accorde parfaitement avec la conclusion de Martin sur le rôle du livre au XVIIe siècle. Il estime que celui-ci était un »combattant, destiné à convaincre autant qu'à instruire ou à informer, qui s'adress[ait] à une élite plus qu'aux masses «213. De la même manière, ce résultat vient confirmer les conclusions de Repgen à propos de la diffusion des publications imprimées lors du congrès de Westphalie dans l'Empire, qui touchaient, elles aussi, un public limité, mais pas uniquement les professionnels 214 .

Tout au long de l'histoire de ces éditions, l'on peut observer certaines évolutions. Elle est marquée par le passage d'une première information sur les principaux résultats des négociations à une approche plus approfondie, qui se traduit par un caractère de plus en plus philologique des éditions. Rappelons qu'une pre-

209 Toscano, History, p. 58, n. 22; ID., Storia, p. 74, n. 2.

$210 \mathrm{Cf}$. le chapitre »Comment mesurer un succès littéraire. Le problème des tirages «, dans:

MARTIN, Le livre français, p. 209-223.

211 Ibid., p. 213.

212 Ibid., p. 214.

213 MARTIN, Livre, pouvoirs et société, p. 965.

214 REPGEN, Westfälische Friede und Öffentlichkeit. 
mière impression avec des notes de bas de page paraît en 1711. On procède de plus en plus à une subdivision plus complexe des articles très longs (notamment en ce qui concerne les articles IV et $V$ de la paix d'Osnabriick) et on signale souvent l'objet des paragraphes en marge du texte. Les éditions prennent de cette façon l'aspect de véritables instruments de travail de juristes, de diplomates, d'historiens.

En ce qui concerne les éditions des traités de Münster et d'Osnabrück en langue française, on peut observer que des notes marginales se trouvent surtout dans les traductions de ce dernier traité publiées par Jean Heiss ${ }^{215}$ et par Guillaume Hyacinthe Bougeant ${ }^{216}$. Mais les éditions de Léonard (1693), de Mauvillon (1749 et 1756), de Scheid (1754) et de Boug d'Orschwiller (1775) comportent aussi des notes marginales ${ }^{217}$. À cet égard, l'évolution des éditions françaises correspond en principe à celle qu'on peut observer dans le Saint Empire romain germanique: en effet, on y procéda à des indications de titres marginaux, surtout depuis l'édition officieuse mayençaise de 1660; ces notes devinrent de plus en plus sophistiquées à partir de la fin du XVII e siècle; elles disparaîtront après 1806 , quand le droit public du Saint-Empire ne fera plus partie des programmes universitaires et ne sera plus appliqué quotidiennement dans le travail des cours de justice et des administrations. En revanche, les notes marginales des éditions françaises n'atteignirent pas le niveau de précision des éditions allemandes et latines publiées dans le Saint-Empire218; en France, la connaissance du droit public allemand n'était pas indispensable à tous les juristes, et cette matière n'occupait pas, dans les universités, la même place qu'en Allemagne. Néanmoins, le fait qu'on ait ajouté aux éditions françaises des notes marginales, quoique moins précises, témoigne de leur utilisation en tant qu'instrument de travail par un certain nombre de lecteurs français.

Par ailleurs, les exemplaires conservés à la Bibliothèque nationale permettent de tirer des conclusions concernant les acquéreurs des éditions des traités, outre l'ancienne Bibliothèque royale: ce sont des ex-libris d'ordres religieux et de savants, surtout de juristes, qui prédominent ${ }^{219}$. Pour ce qui est de l'intérêt qu'on porte à ces traités, c'est l'actualité politique qui le cède aux sciences (histoire et droit), et la curiosité à l'égard de l'Allemagne qui prend le relais de l'histoire

215 HeIss, Histoire de l'Empire; ces notes marginales ne se trouvent que dans les trois premières éditions de 1684 , de 1685 et de 1694.

216 BougEant, Histoire du traité de Westphalie, éditions de 1744, 1751 et 1767.

217 Pour cette dernière édition, voir ci-dessous.

218 Une particularité intéressante: dans sa traduction de l'»Histoire du traité de Westphalie« de Bougeant, parue de 1758 à 1760 , Rambach reprit les titres marginaux très précis que Meiern avait inventés en 1738 (voir ci-dessus) et les traduisit en allemand, cf. BougEANT, Historie des dreyBigjährigen Krieges. Pour les notes marginales des éditions latines et allemandes des traités de Westphalie, cf. Antje Oschmann (éd.), Acta Pacis Westphalicae, série III, section B, vol. 1: Die Friedensverträge mit Frankreich und Schweden, t. 3: Materialien zur Erschließung und Register, Münster 2007 (APW III B 1/3), pour Rambach p. 5, n. 32.

219 Cf. notre bibliographie. 
nationale. C'est pourquoi, après la prédominance du traité conclu par la France, celui de Münster, jusqu'aux années 1680 , c'est le traité de paix d'Osnabrück qui gagne de plus en plus de terrain à partir de 1684. D'abord, il se trouve souvent imprimé à côté de celui de Münster, surtout dans des ouvrages centrés sur la paix de Westphalie (chez Heiss et Bougeant); puis il apparaît seul, comme loi fondamentale de l'Empire, dans des traités sur le droit public allemand (chez Mauvillon et Scheid). La vision de l'Allemagne et de son droit public, qui se saisit à travers l'explication fournie par les commentateurs de ces traductions, montre que les principaux termes juridiques employés dans les traités de Westphalie étaient soumis à des interprétations très divergentes tout au long des XVIIe et XVIIIe siècles. Les opinions des publicistes différaient surtout en fonction de leur interprétation de la forme du gouvernement du Saint-Empire et de ses institutions centrales. En particulier, le ban de l'Empire et la capitulation impériale faisaient l'objet, dans ce contexte, de vives controverses 220.

Or, les traductions de l'article VIII du traité d'Osnabrück ne sont guère différentes $^{221}$. Nous avons déjà vu que la paix de Westphalie reconnut la »Landeshoheit (ius territoriale) des états de l'Empire en tant que pouvoir seigneurial territorial qui dépassait les droits régaliens particuliers ainsi que les titres juridiques traditionnels et qui était indépendant par rapport à eux «222. En effet, l'article VIII du traité d'Osnabrück stipule223:

Et afin de pourvoir à ce que dorénavant il ne naisse plus de différends dans l'Etat politique, tous et chacun des Electeurs, Princes et Etats de l'Empire Romain seront tellement établis et confirmés en leurs anciens droits, prérogatives, libertés ${ }^{224}$, priviléges, libre exercice du Droit Territorial, tant au Spirituël qu'au Temporel ${ }^{225}$, Seigneuries, Droits régaliens, et dans la possession de toutes ces choses, en vertu de la présente Transaction, qu'ils ne puissent jamais être troublés de fait par qui que ce soit, sous quelque prétexte que ce puisse être.

Il convient de souligner que toutes les traductions des traités, malgré certaines différences de moindre importance et quelques lacunes dont il a déjà été question, sont d'accord sur la traduction des principaux termes juridiques. Surtout, on note qu'aucune d'entre elles ne traduit »ius territoriale« par »souveraineté«. Tous les

$220 \mathrm{Ce}$ sont les différentes solutions apportées à ces questions que nous allons analyser dans la troisième partie de ce livre, en étudiant, en particulier, un certain nombre de juristes et d'historiens, tels que Heiss, qui se fondent notamment sur l'interprétation de l'article VIII du traité d'Osnabrück.

${ }^{221}$ Le problème des clauses concernant les cessions territoriales est abordé au chapitre sur les frontières de l'Empire.

222 Böckenförde parle, en allemand, de la confirmation de la »Landeshoheit (ius territoriale) der Reichsstände als einer die einzelnen Hoheitsrechte und überkommenen Rechtstitel transzendierenden, ihnen gegenüber selbständigen territorialen Herrschaftsgewalt «, cf. BöCKENFÖRDE, Bündnisrecht, p. 454.

223 Art. VIII,1 IPO $=\S 62$ IPO. Nous citons la traduction de SCHEID, Traité sistématique, t. IV, p. 230. Cf. aussi le texte latin: OsChmanN, APW III B 1/1, p. 19, 130.

224 En latin, au singulier: »libertate«; ibid.

225 En latin: »libero iuris territorialis tam in ecclesiasticis quam politicis exercitio«; ibid. 
traducteurs parlent de »droit territorial ${ }^{226}$, la »Gazette« de »droit de leur territoire ${ }^{227}$.

\subsection{Les dernières traductions de l'Ancien Régime}

Même si la période étudiée s'achève en 1756, il convient de signaler encore quelques publications des traités de Westphalie parues en France après cette date, et surtout leur dernière version manuscrite établie par un interprète des Affaires étrangères, datée de $1777^{228}$. Très certainement, l'Alsace, terre de langue allemande, ne vit paraître une traduction française du traité de Münster dans une de ses villes, Colmar, que vers la fin de l'Ancien Régime, en 1775. C'est l'une des toutes dernières éditions françaises de la paix de Westphalie. Elle fut publiée dans les »Ordonnances d'Alsace « éditées sous la direction de François-Henri Boug d'Orschwiller (1722-1775), qui était devenu conseiller au conseil souverain d'Alsace en 1747 et premier président en $1768^{229}$. Le fait même qu'on y ajoutât les articles V et VII du traité d'Osnabrück, qui avaient fixé la nouvelle constitution religieuse de l'Allemagne, témoigne des liens qui, du moins jusqu'à la capitulation de Strasbourg, subsistaient encore entre l'Alsace et l'Empire; de même, Laguille, dans son histoire de l'Alsace, reproduit certains paragraphes de l'article $\mathrm{V}^{230}$. C'est qu'on considéra qu'il constituait un règlement (au moins partiellement) applicable en Alsace dans la période recouverte par ce tome des »Ordonnances «, c'est-à-dire la deuxième moitié du XVIIe siècle. De Boug choisit la paix de Münster dans la »Nouvelle édition« de l'»Histoire de l'Empire « de Heiss, et les extraits de la paix d'Osnabrück de Scheid. Autant qu'on puisse le dire en l'état actuel des connaissances, nous devons la toute dernière traduction imprimée publiée sous l'Ancien Régime à Simon Jacquet; comme les »Ordonnances «, elle parut en Alsace, plus précisément à Strasbourg. Jacquet publia, en 1782, un ouvrage en six volumes sur le »Droit public d'Allemagne«; les deux derniers tomes repro-

226 Cf., par exemple, HeIss, Histoire de l'Empire (1685), t. III, p. 106 (dans la traduction de l'art. VIII,1 IPO). Certes, il y a plusieurs variantes, comme »droit de territoire (ibid., p. 105, dans la traduction de l'art. VII,1 IPO), etc.

227 Gazette de France n ${ }^{\circ} 174$ (1648), p. 1550, art. 38 selon la numérotation de cet imprimé.

228 Pour la datation exacte de ce document, voir ci-dessous.

229 Henri François Boug [D'ORSCHWILLER], Ordonnances d'Alsace [avant-titre]. Recueil des édits, déclarations, lettres patentes, arrêts du Conseil d'État et du Conseil souverain d'Alsace, ordonnances \& règlemens concernant cette province [...], 2 vol., Colmar 1775; traité de Münster: t. I, p. [I]-XXI (texte divisé en cent vingt paragraphes; notes marginales indiquant les principaux objets du traité); traité d'Osnabrück (extraits): ibid., p. XXII-XXXVI (préambule, incipit de l'article premier, articles V et VII; notes marginales indiquant les principaux objets des articles concernés). Pour ce personnage, cf. T. DE MOREMBERT, article „Boug d'Orschwiller (François-Henri)«, dans: Dictionnaire de biographie française, t. VI (1954), col. 1286; Jean-Marie SchmiTt, article »de Boug, François-Henri«, dans: NDBA, t. 4 (1984), p. 314.

230 LAGUILLE, Histoire de la province d'Alsace, édition in-folio, troisième partie, p. 159161: $\S \S 73-75,79,87-88$ IPM et art. V,1, V,16 et V,25-26 IPO; en latin, cf. OsChMANN, APW III B 1/1, p. 22-24, 26, 110-111, 114, 116-118. 
duisent des lois impériales et des traités passés par l'Empire. La publication de lois et de traités en annexe d'un ouvrage sur le droit public du Saint-Empire était une tradition bien ancrée chez les auteurs de langue française, depuis Jean Heiss. En 1787, Jacquet fit réimprimer cette collection de lois et de traités, sous le titre de »Recueil precieux et unique des loix fondamentales de l'Empire «231. Il s'agit d'une véritable réimpression des lois et des traités publiés à la fin du »Droit public d'Allemagne «, en 1782; le texte est identique ${ }^{232}$. Une note ajoutée au tome premier du »Recueil precieux « précise que cette nouvelle publication du seul appendice du »Droit public d'Allemagne« répondait à une demande du public 233 . En France, on avait donc toujours besoin de bonnes éditions françaises des lois du Saint-Empire. Cependant, ces éditions de 1782 et de 1787 n'offrent pas de traductions nouvelles: Jacquet a en effet tiré la version du traité de Münster de Heiss et pris celle de la paix d'Osnabrück de Scheid; au traité d'Osnabrïck il a ajouté la ratification par l'empereur et celle de la reine de Suède. À la fin de ce traité, Jacquet fait des »observations «, renvoyant à l'»Histoire de l'Empire« de Heiss pour trouver plus d'informations et d'autres documents; il est ainsi possible de conclure qu'il a utilisé l'édition in- $8^{\circ}$ de $1731^{234}$. D'après nos recherches, ce fut en 1787 la dernière publication des traités de Westphalie en langue française sous l'Ancien Régime $^{235}$. Avec la Révolution en 1789 et la disparition du Saint-Empire en 1806,

231 JACQUET, Droit public d'Allemagne; les traités de Westphalie se trouvent au t. V, p. 39102 (Münster), 103-238 (Osnabrück); observations sur les deux traités, p. 238-240; [ID.], Recueil precieux; les traités de Westphalie se trouvent au vol. I, avec la même pagination qu'en 1782.

232 En effet, ce »Recueil precieux « reproduit la fin du »Droit public d'Allemagne « de 1782; le $t$. I contient, plus précisément, le vol. IV, p. 246-256 du »Droit public «, avec une pagination différente; le vol. IV, p. 257-422 du "Droit public «, avec la même pagination qu'en 1782; et le t. V, jusqu'à la p. 365, sans que la pagination de 1782 ait été changée; le t. II contient le reste du vol. V du »Droit public« et le texte intégral du vol. VI, sans modification de la pagination de 1782. Le début du premier tome du »Recueil precieux « a donc sa propre pagination, mais la page 16 est suivie de la page numérotée 257 (comme en 1782).

233 Cette note excuse la pagination bizarre du »Recueil precieux«, qui était vendu non seulement à Strasbourg, mais aussi à Paris, chez Musier, cf. Recueil precieux, t. I, p. 16, note. 234 En effet, ses observations renvoient à »Heiss, Histoire d'Allemagne. tom. VII. p. 331 \& suiv.«, pour connaître les noms des signataires du traité (JACQUET, Droit public d'Allemagne p. 238), et à »l'Histoire de l'Empire de Heiss. tom. VII. p. 334. \& suiv.« au sujet des recès d'exécution de la paix de Westphalie, signés à Nuremberg en 1650, de la Ligue du Rhin de 1658 et de la protestation du pape contre la paix de Westphalie (ibid., p. 240). On relève une imprécision: la protestation du pape se serait seulement dirigée contre le traité d'Osnabrück. Cf. HeIss, Histoire de l'Empire (1731), édition in-8 $8^{\circ}$, t. VII; dans les autres éditions, la tomaison ou la pagination est différente.

${ }^{235}$ Un ouvrage intitulé Recueil precieux [...] est aussi indiqué par DuCHHARDT, Bibliographie, p. 16, $n^{\circ} 186$, mais avec la mention »Traduit par P. Jacquet « (qui ne se trouve pas sur le frontispice de l'édition de 1787) et l'année de publication 1788; de plus, la paix de Westphalie se trouverait au vol. II (rappelons que dans l'édition de 1787, les traités se trouvent au vol. I). En revanche, l'exemplaire de 1787 lui est inconnu. Pas plus que Duchhardt et ses collaborateurs, qui précisent qu'ils n'ont pas vu ce livre, nous n'avons pu retrouver cette édition qui daterait de 1788, à supposer qu'elle n'ait été confondue avec celle de 1787. 
l'intérêt que les Français portaient à ces traités allaient diminuant, à en juger par l'absence d'éditions intégrales françaises postérieures ${ }^{236}$.

Au dépôt des archives, précurseur des actuelles archives diplomatiques du ministère des Affaires étrangères, on s'intéressa dès la deuxième moitié du XVIIe siècle à l'étude des traités de Westphalie et des négociations qui y avaient abouti. Leur étude concernait aussi les aspects techniques des traités. Par exemple, le volume Allemagne, quarante et unième de la série »Mémoires et documents«, comprend une brève note ${ }^{237}$ qui explique la division en articles ou en paragraphes du traité de Münster ${ }^{238}$. C'est surtout à Adamus Adami que l'auteur de la note rédigée au dépôt des Affaires étrangères s'est rapporté et c'est plus précisément dans l'édition de $1698^{239}$ qu'il a puisé la division du traité de Münster.

Or, au dépôt des Affaires étrangères, on n'a pas seulement eu le souci d'avoir à disposition une division claire et non ambiguë du traité de Münster afin d'en permettre des citations, mais on a encore pris le soin, dans la deuxième moitié du XVIII ${ }^{e}$ siècle, d'en établir une nouvelle traduction. Jean-Jacques de Zabern, interprète des Affaires étrangères, en fut chargé ${ }^{240}$. Le personnage n'a (autant que nous sachions) jamais attiré l'attention des historiens, mais on peut trouver des informations sur sa vie dans son dossier personnel aux archives ${ }^{241}$. Toutefois, ce dossier ${ }^{242}$ ne donne pas beaucoup de renseignements sur la vie et sur le travail de cet interprète ${ }^{243}$; il réserve pourtant une surprise: Zabern, gravement malade, était

236 Édition latine en 1893 par VAST, Traités, t. I.

237 »Sur le Traité de Munster «; AE, MD All. 41 fol. 89. Note non numérotée, qui doit être certainement attachée à la pièce suivante. En effet, cette note et le document no 9 du volume concernent la paix de Westphalie.

238 Pour cette question, cf. OsChMANN, APW III B3/2, »Initien und Gliederungen des IPO und des IPM «, p. 685-710.

239 Adam Adami, édition de 1698; cf. A[damus] A[DAMI], Arcana Pacis Westphalicae [...], Francfort/M. 1698; le texte latin du traité de Münster se trouve, en annexe, p. 65-97. La deuxième édition de cet ouvrage fut publiée en 1707. Le traité de Münster ne se trouve pas dans l'édition des »Arcana Pacis« d'Adami, établie en 1737 par Meiern, qui devait lui-même éditer ce traité en 1738; cf. Adam ADAMI, Relatio historica de pacificatione Osnabrvgo-Monasteriensi ex avtographo avctoris restitvta atqve actorvm pacis vestphalicae testimoniis avcta et coroborata ac cvrante Joanne Godofredo DE MEIERN [...], Leipzig 1737.

$240 \mathrm{Cf}$. sa version du traité conservée dans le volume AE, MD All. 41 fol. 90, 91-121: "Traduction du traité de paix de Munster. Signé le 24 octobre 1648. Par Jean-Jacques von Zabern, interprète des Affaires étrangères. En 1777 « (titre fol. 90).

241 Tout en étant lacunaires, les informations qu'on peut trouver sur ce personnage permettent de mieux connaître la situation personnelle d'un interprète et traducteur de documents diplomatiques au XVIIIe siècle, et de comprendre dans quelle mesure l'État suppléa à ses besoins et à ceux de sa famille au moment où il devint invalide.

242 AE, Personnel, $1^{\text {re }}$ série, vol. 69 fol. 208-215.

243 Par ailleurs, le nom »von Zabern« (de Zabern [doit-on ou peut-on traduire par »de Saverne «? - dans les documents consultés, on ne le fait jamais]) apparaît seulement sur la traduction des traités de Westphalie, mais jamais dans ce dossier personnel, où l'on dit seulement $\gg Z a b e r n «$. D'ailleurs, il est à noter que l'inventaire de cette série indique comme nom du traducteur »J. van Zabern «, cf. Inventaire sommaire des archives du département des Affaires étrangères. Mémoires et documents. Fonds divers [dressé par le bureau historique de la 
déjà parti à la retraite en 1776 , de sorte que sa version des traités de Westphalie ne peut pas dater de 1777; il mourut d'ailleurs en 1777 ou au début de 1778. Les documents qui se trouvent dans son dossier personnel concernent uniquement la pension que le roi lui accorda lorsqu'il ne fut plus en mesure d'accomplir ses tâches au ministère, et la pension qui fut accordé à sa veuve après sa mort. De ces documents, il découle cependant que Zabern avait travaillé dix ans durant comme interprète au ministère des Affaires étrangères. La dernière note conservée dans ce dossier personnel concerne directement ces versions de la paix de Westphalie et leur reclassement ${ }^{244}$. Or, cette traduction n'est pas tout à fait complète. En effet, Zabern n'a pas traduit le préambule du traité, mais il renvoie, à moins qu'il ne s'agisse d'un autre collaborateur du dépôt, dans une note additionnelle au-dessus de la traduction de l'article Irer, à l'édition de Bougeant ${ }^{245}$. On peut conclure de l'indication du volume et de la pagination que le rédacteur de cette note se réfère soit à l'édition de 1744 , et plus précisément au tirage in-12246, soit à celle de $1751^{247}$, mais pas à la dernière édition, qui date de $1767^{248}$. Pour d'autres articles, la traduction renvoie simplement au traité d'Osnabrück qui a la même teneur ${ }^{249}$. La division de cette traduction est partiellement calquée sur celle de la paix d'Osnabrück, car, outre une division en cent vingt paragraphes, le traducteur a encore ajouté une division en dix-sept articles ${ }^{250}$. On trouve, dans le même volume, une traduction partielle de la paix d'Osnabrück ${ }^{251}$. À la fin de ce traité, dont la traduc-

division des archives], Paris 1892 , p. 16. Suite à cette indication, nous avons attribué la traduction à »Jean-Jacques van Zabern« dans: BRAUN, Die »Gazette de France«, p. 293, n. 51. 244 Note intitulée: »Zabern «; sans date; AE, Personnel, 1're série, vol. 69 fol. 215: »[Les] Traductions des traités de Munster et d'Osnabruk par le sieur Zabern sont placées dans la correspondance de Vienne à la suite du volume timbré: 1648-1659«. Mentions à la fin, de la même main: »à vérifier « et »au 13«. Il s'agit aujourd'hui du vol. 17 de la série Correspondance politique, Autriche, qui est essentiellement composé de papiers Mazarin et de Chavigny.

245 AE, MD All. 41 fol. 91: „Le préambule du traité, qui manque ici, se trouve traduit dans l'ouvrage du père Bougeant, jésuite, intitulé: >Histoire de Westphalie‘, Tome VI, page 215 « (le titre de l'ouvrage est souligné dans l'original).

246 Bougeant, Histoire du traité de Westphalie (1744, in-12). Dans l'édition in-4º, publiée la même année, le traité de Münster commence t. II, p. 507.

247 ID., Histoire du traité de Westphalie (1751).

248 ID., Histoire des guerres et des negociations (1767). Dans cette édition, le traité de Münster commence t. III, p. 507.

249 Cf. AE, MD All. 41 fol. 96-99', 117', 119'-120' où de très nombreux articles sont "à copier « sur le traité d'Osnabrück, et la note ajoutée à la fin de cette traduction: »Il faut copier le reste sur la paix d'Osnabruck en mettant vers la fin à la place du mot $>$ Osnabruck ‘ celui de $>$ Munster « (fol. 191; les noms de lieux sont soulignés dans l'original). Cette remarque est un peu simplificatrice, car les articles de la paix d'Osnabrück n'équivalent pas toujours au mot près à la paix de Münster, cf. les différenciations chez OschmanN, APW III B 1/1, p. 2 et p. 96.

${ }^{250} \mathrm{Ce}$ nombre correspond à la numérotation de l'original du traité d'Osnabrïck, cf. OSCHMANN, APW III B 1/1, document $n^{\circ} 18$, p. 95-170.

251 AE, MD All. 41 fol. 122, 123-127: »Paix d'Osnabruck. Art. 1 et 2, 3. 1648. Empereur, Empire et Suède " (titre fol. 122); ibid., fol. 128, 129-150: "Article IV de la paix d'Osnabruck « (titre fol. 128); ibid., fol. 151-188: „Paix d'Osnabruck. Article V « avec la note, ajou-

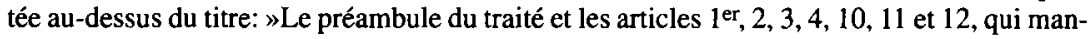


tion comporte seulement quelques lacunes (la plus importante étant l'omission entière de l'article XI), le traducteur reproduit la liste des signataires et la clause qui oblige tous les états non signataires du traité à son observation ${ }^{252}$. D'après l'homogénéité paléographique des deux traductions des traités de Münster et d'Osnabrück on peut conclure qu'ils sont contemporains, puisque c'est une seule main qui a écrit presque tout le texte. Cela rend aussi probable l'hypothèse selon laquelle Zabern serait le traducteur non seulement du premier, mais aussi du deuxième traité. Toutes ces copies comportent seulement quelques corrections ultérieures, qui sont mineures et ne touchent pas la terminologie du droit public impérial ou du droit des gens; elles sont un peu plus nombreuses dans la traduction de l'article V du traité d'Osnabrück qui devait être l'un de plus difficiles à traduire en français, car concernant le droit de religion propre à l' Allemagne, pierre d'achoppement pour quasi tous les traducteurs. En général, il n'y a aucun commentaire; les seules exceptions concernent les articles V et VII de la paix d'Osnabrück. On peut conclure de ces annotations que le droit de religion allemand et la situation des protestants furent plus difficiles à comprendre en France que les clauses politiques qui ne font pas l'objet de telles explications ${ }^{253}$. La traduction de l'article VIII du traité d'Osnabrück, mais aussi des clauses de cession dans le traité de Münster témoignent de la valeur de cette version en général et de son indépendance face aux traductions antérieures 254 .

quent ici, se trouvent traduits dans l'ouvrage du père Bougeant, intitulé: Histoire de Westphalie, Tome VI, p. 281 «. C'est encore aux éditions de 1744, tirage in-12, ou de 1751 (cf. cidessus) que cette indication de volume et de page doit renvoyer. AE, MD All. 41 fol. 188'189: »Article VI« (du traité d'Osnabrück); ibid., fol. 189-191': "Article VII«; ibid., fol. 191'-195: »Article VIII«; ibid., fol. 195-196': »Article IX«; ibid., fol. 197, 198-208': "Article X de la paix d'Osnabruck « (titre d'après fol. 197); ibid., fol. 210, 211-221: »Article XI de la paix d'Osnabruck « (titre d'après fol. 210); ibid., fol. 222: »Article XII« (le titre seul sans qu'on y ait ajouté le texte de l'article même); ibid., fol. 223, 224-234: "Article XIII de la paix d'Osnabruck « (titre d'après fol. 223); ibid., fol. 235, 236-238: »Article XIV de la paix d'Osnabruck « (titre d'après fol. 235); ibid., fol. 239, 240-249': "Article XV de la paix d'Osnabruck « (titre d'après fol. 239); ibid., fol. 250, 251-265, 266, 267-275: »1648. Article XVI de la paix d'Osnabruck. Article XVII et fin de la paix d'Osnabruck « (titre d'après fol. 250; l'article XVII a aussi son titre propre fol. 266).

252 Ibid., fol. 273'-275.

253 En revanche, le nombre de corrections apportées à la traduction ne peut pas en être une preuve, puisqu'il est possible que des essais préparatoires aient été perdus.

254 On note, par exemple, qu'à l'article VIII,1 IPO, Zabern utilise le terme de »supériorité territoriale « pour traduire le passage latin »libero iuris territorialis tam in ecclesiasticis quam politicis exercitio«; les traducteurs antérieurs s'en étaient tenus à l'expression latine figurant dans l'original du traité (qui signifie littéralement »droit territorial «, et c'est de cette manière que Heiss, en 1684, et Scheid, en 1754, l'ont traduite). Mais au XVIII' siècle, l'usage du terme »superioritas territorialis« s'était imposé dans les ouvrages latins des juristes allemands, tandis qu'en France, les spécialistes du droit allemand employaient fréquemment le concept de »supériorité territoriale«. Zabern modernise donc la terminologie de cet article tout en respectant scrupuleusement son sens. Sa traduction de l'expression "in statu politico « se distingue également des versions de Heiss et de Scheid qui la traduisent littéralement: »dans I'Etat politique«; pour sa part, Zabern accorde au mot status le sens moderne du concept »État«, puisqu'il parle à ce propos »du gouvernement de l’État«. De toute évidence, 


\section{Les traductions françaises de la Bulle d'or (1356)}

Comme les Français montraient beaucoup d'intérêt non seulement pour la paix de Westphalie, mais aussi pour la Bulle d'or, de nombreux manuscrits contiennent des résumés de cette loi fondamentale ${ }^{255}$. La Bulle d'or, acte nommé d'après le sceau en or massif qui y était attaché, fut »la prémiére des Loix fondamentales, qui concernent l'Empire Germanique« et, même au XVIII' siècle, elle était toujours censée »être perpétuelle «256. Cela ne signifie pas qu'elle a été soustraite à toute modification ${ }^{257}$. En français, Abraham de Wicquefort décrivait minutieuse-

Zabern veut se libérer des contraintes d'une traduction littérale pour adapter la teneur de sa traduction à la terminologie des années 1770 . Mais, parfois, il corrige aussi les traductions imprécises de ses prédécesseurs. Contrairement à Heiss et à Scheid, il traduit correctement "libertate« par "liberté« (Heiss et Scheid ont mis ce mot au pluriel); cf. AE, MD All. 41 fol. 191'-195 (art. VIII IPO), ici fol. 191'-192 (\$ 1). Cette modernisation terminologique se manifeste également au deuxième paragraphe quand Zabern traduit "nomine publico" par l'expression »au nom du Corps germanique «, une notion très à la mode au XVIII siècle (ibid., fol. 192); Heiss et Scheid l'ont traduite mot-à-mot: »au nom du public«. Cf. HeIss, Histoire de l'Empire (1685), t. III, p. 106-109; SCHEID, Traité sistématique, t. IV, p. 230234. Pour le texte latin de l'article VIII, cf. OschmanN, APW III B 1/1, p. 130-131, ici p. 130. En ce qui concerne la notion moderne d'État, Zabern l'adopte aussi à l'art. V,1 IPO, où il traduit »formae reipublicae « par »Constitution de l'État « (AE, MD All. 41 fol. 151'); conformément à l'original latin, Heiss et Scheid ont gardé le terme de »respublica«: »l'état de la République« (HEISS, Histoire de l'Empire [1685], t. III, p. 74; ScHEID, Traité sistématique, t. IV, p. 181-182, ici p. 182). Malgré cette modernisation du langage politique, on constate que la terminologie relative à la Constitution du Saint-Empire n'a guère évolué. Toutefois, on voit que certains termes techniques français ("supériorité territoriale«), voire latins, sont véritablement enracinés dans le langage des spécialistes français du droit public allemand. L'usage du terme "supériorité territoriale « est général dans sa traduction de la paix de Westphalie; il l'utilise aussi à l'art. VII,l IPO pour traduire »iuris territorialis« (ibid., fol. 190'), alors que, sous l'Ancien Régime, aucune traduction imprimée de la paix de Westphalie n'utilise le terme de »supériorité territoriale«.

255 Cf., par exemple, BNF, F.fr. 18988 (»Recueil sur l'Empire d'Allemagne, sur le droit germanique et sur les élections des empereurs «), II fol. 61-99 et fol. 104-110: résumé français des chapitres I à VIII [ $\$ 1$ 1] de la Bulle d'or. Ce manuscrit semble dater du début du XVIII siècle. Torsten Hiltmann a bien voulu nous signaler le fait qu'un corpus médiéval de documents relatifs à la charge des hérauts contient aussi une version française de la Bulle d'or; il aurait été constitué à Metz (copies de la bulle, selon Hiltmann: Metz, bibliothèque municipale, ms. 595 fol. 271-285'; ibid., ms. 855 fol. 47-63'; Berne, Burgerbibliothek, ms. A 280 fol. CXIII'-CXX').

256 Heiss, Histoire de l'Empire (1733, édition in- $\left.8^{\circ}\right)$, t. VI, p. 371-381: »Remarques, Sur les Loix \& Coutumes de l'Empire «, ici p. 373-374 (t. II, p. 350-353, citations p. 351, dans l'édition in-4 $4^{\circ}$ ). Ces remarques doivent très certainement être attribuées aux éditeurs hollandais ou à Vogel, grand juge des gardes suisses et responsable de l'édition de l'»Histoire de l'Empire " publiée en 1731 et peut-être de celle de 1733, puisqu'elles ne se trouvent pas dans les éditions antérieures à 1733. Selon Soisson, il s'agit même de »l'une des premières Constitutions de l'histoire de l'Europe «, Sorsson, Charles Quint, p. 52.

257 Le continuateur de Heiss note dans Heiss, Histoire de $1^{\prime}$ Empire (1733, édition in- $8^{\circ}$ ), t. VI, p. 379: »non seulement la nature des Loix fondamentales admet les changemens, qui tournent au bien de la République, lorsqu'ils sont faits par ceux qui ont le pouvoir Legislatif; la Bulle d'or elle-même \& les autres Loix de l'Empire autorisent de pareils changemens«. 
ment l'original de ce document précieux, conservé dans l'hôtel de ville de Francfort-sur-le-Main, et en particulier son sceau d'or, qu'il interprétait comme »une marque de Souveraineté en la personne de l'Empereur « ${ }^{258}$. Les règlements que la Bulle d'or contenait dépassaient largement le seul champ de l'élection impériale et fixaient certains principes fondamentaux au gouvernement général de l'Empire 259 . L'»Histoire de l'Empire « précise que, outre cette Bulle d'or de 1356, un deuxième diplôme de l'empereur Charles IV porte ce titre, à savoir la »Bulle Brabantine « donnée en 1349 en faveur de Jean duc de Brabant ${ }^{260}$. Toutefois, pour Wicquefort, la bulle de 1356 était la Bulle d'or »par excellence«, puisque cet édit avait un très grand avantage »sur tous les autres, servant comme de Pragmatique Sanction, ou plûtôt comme de Loy fondamentale à l'Empire, de base à la grandeur des Electeurs, \& de lustre à la dignité Electorale ${ }^{261}$. Cette loi fondamentale de 1356 , qui fixait les règles pour l'élection des rois des Romains et des empereurs ainsi que la condition juridique des électeurs du Saint-Empire ${ }^{262}$, nécessite des explications plus longues, puisque l'histoire de sa traduction a commencé bien avant 1648. Mais, en réalité, le premier ouvrage sur l'élection impériale fut l'cuvre d'un Italien traduite en français.

Le premier auteur français à publier un ouvrage sur l'élection impériale fut en effet Le Secq. La première édition de son livre sur l'élection impériale, qui com-

Parmi les changements survenus depuis 1356, cet auteur note en particulier la création de deux dignités électorales supplémentaires. Il évoque aussi le fait que, à son époque, plusieurs actes écrits étaient rédigés en allemand au lieu du latin prévu dans la Bulle d'or.

$258 \mathrm{Cf}$. [Abraham DE WICQUEFORT], Discours historique de l'élection de l'Empereur [...], Rouen 1711; ici chapitre VIII (p. 130-150), »Diverses Remarques sur la Bulle d'Or« (citation p. 131). La première édition de cet ouvrage datait de 1658 (l'année de l'élection de Léopold Ier). Cette réédition fut publiée à l'occasion de l'élection de Charles VI (1711).

259 En France, on lisait des publications qui soulignaient cette fonction de la Bulle d'or comme loi fondamentale générale de l'Empire germanique. Il s'agissait non seulement d'auteurs connus et d'ouvrages de jurisprudence, mais aussi de publications de circonstance. Cf., par exemple, un imprimé français publié à titre anonyme au lendemain de la mort de l'empereur Léopold Ier: „Memoire contenant les constitutions de l'Empire sur la Bulle d'Or, avec les droits des électeurs \& du Corps germanique pour l'élection d'un Empereur. Suivant la tradition d'allemand en françois. Sur l'imprimé, à Ratisbonne en Allemagne«, 1705. Cette petite brochure constitue un récit partial du règne de Léopold $\mathrm{I}^{\text {er }}$ et lui reproche des contraventions à sa capitulation impériale et la mise en place d'un pouvoir absolu. En revanche, l'auteur loue la sagesse de l'empereur Charles IV, qui promulgua »la Bulle d'Or, si respectée autres fois en Allemagne, \& qui est dans cette Nation la Loy que l'on consulte ordinairement, puisque l'Auteur de cette Bulle avoit prit [sic] soin d'y rassembler toutes les choses qui pouvoient regarder le gouvernement de l'Empire [...] \& il est certain que dans un gouvemement moins absolu, l'inobservation d'un seul chef de cette loy eust rendu une Election nulle « (ibid., p. 27-28). Pour un résumé de la capitulation de Léopold Ier, cf. ibid., p. 14-27.

260 Cf. HEISs, Histoire de l'Empire (1733, édition in-8 $8^{\circ}$ ), t. VI, p. 380.

261 [WICQUEFORT], Discours historique de l'élection de l'Empereur, Rouen 1711, p. 130.

262 Le continuateur de Heiss signale cependant des usages qui ne font pas partie des lois écrites de l'Empire. Dans ce contexte, il évoque la règle selon laquelle les électeurs ne pourraient pas élire un empereur étranger: »De ce nombre on peut mettre, la Régle de n'élever à l'Empire qu'une Personne d'Origine Allemande \& de naissance illustre «; HeIss, Histoire de l'Empire (1733, édition in-80), t. VI, p. 372. 
prend deux parties, fut publiée pour la première fois à Paris, en 1612 et $1613^{263}$, et réimprimée dans la capitale, en $1633^{264}$. Ce livre offre, dans sa deuxième partie, un résumé de la Bulle d'or. Il s'insère dans la tradition française qui s'intéresse surtout au problème de l'élection impériale, tandis que quasi tous les autres problèmes de la Constitution allemande se trouvèrent d'abord, du moins jusqu'à la paix de Westphalie, relégués au deuxième rang. C'est aussi le seul véritable livre français sur un aspect du droit impérial, avant la paix de Westphalie. Mais, comme nous venons de le dire, Le Secq, sur lequel on ne sait d'ailleurs pas grand-chose, n'a pas écrit lui-même son ouvrage. En effet, il traduit seulement un livre publié par un Italien peu de temps avant: Girolamo Canini d'Anghiari, qui avait publié, en 1612, sa »Sommaria historia della Elettione, e coronatione del Rè De' Romani«, à laquelle il avait joint "vn Compendio della sudetta Bolla di Carlo Quarto Imper[atore] «, c'est-à-dire un abrégé de la Bulle d'or de Charles IV, empereur romain germanique ${ }^{265}$. Le problème des sources italiennes de la littérature française sur le Saint-Empire n'est pas sans importance; étant donné qu'au XVIe siècle les Vénitiens passaient pour les meilleurs connaisseurs des différents pays d'Europe, il n'est pas étonnant de voir qu'encore au début du XVIIe siècle les Français faisaient toujours confiance aux Italiens pour connaître les pays limitrophes. Mais cette influence a sensiblement diminué en 1648. Cependant, l'influence que Canini exerça sur les débuts de la littérature française relative au droit de l'élection impériale est tout à fait considérable ${ }^{266}$. En 1612, ce fut à l'occasion de l'élection de Matthias que, pour la première fois, il publia son traité sur l'élection impériale, accompagné des résumés de la Bulle d'or et d'une capitulation impériale qui semble être celle de Charles Quint ${ }^{267}$. Dans l'Empire, on avait également publié la Bulle d'or pour la même occasion ${ }^{268}$. La vie d'I. Le Secq, traducteur de Canini, est moins bien connue que celle de l'Italien. Même le prénom n'est pas connu. Comme les lettres "I« et "J« ne sont pas distinguées selon les critères contemporains dans les publications de cet auteur, la solution la plus simple serait de supposer qu'il s'appelait Jean. Mais, dans l'état actuel des connaissances, cette hypothèse ne peut pas être vérifiée. Selon la préface de l'édition française, Canini et Le Secq se connaissaient personnellement, ayant passé sept ans ensemble dans divers

263 I. LE SECQ, Histoire de l'élection et covronnement du Roy des Romains [.. .], Paris 1613; [ID.], Abregé de la bvlle doree de l'Emperevr Charles IIII. [...], Paris 1612.

264 [ID.], Histoire de l'élection et covronnement dv Roy des Romains [...], Paris 1633; dans les deux éditions, la Bulle d'or se trouve p. 1-29 de l'»Abrégé«.

265 [Girolamo Canini D'ANGHIARI], Sommaria historia della elettione, e coronatione del Rè de' Romani. Compresa in vn breve discorso sopra la Bolla d'oro di Carlo Quarto Imperadore [...], Venise 1612; [ID.], Compendio della bolla di Carlo Qvarto Imperadore [... ], ibid. 1612. 266 Cf. Guido BRAUN, Ein italienischer Blick auf Kaiserwahl und Kurfürstenrat im frühen 17. Jahrhundert und seine Vermittlung in Frankreich: Girolamo Canini und I. Le Secq, dans: Frühneuzeit-Info 17 (2006), p. 70-93.

${ }^{267}$ L'auteur parle seulement de la capitulation au singulier comme s'il n'en existait qu'une seule.

268 Cf., par exemple, Avrea Bvlla Karoli IIII. Rom. Impeatoris: ex archiuo illustrissimi princ. electoris palatini, Spire 1611. 
pays d'Europe ${ }^{269}$. D'après Cioranescu270, I. Le Secq est également l'auteur de deux petites brochures: une "Egloga. A Madama «271, signée Le Secq, et l'»Ode saphiqve rimée \& mesurée. A la Reyne ${ }^{272}$, avec la même indication d'auteur. Ces deux textes littéraires sont en réalité des panégyriques dédiés à Marie de Médicis. Mais, pour notre étude, ils n'ont qu'un intérêt mineur, si ce n'est qu'ils montrent l'ancrage de la pensée de notre auteur, qui était dévoué à la fois à la monarchie française et au clergé ultramontain.

L'histoire de l'élection impériale fut d'abord publiée en 1613 en français. Bien que la deuxième partie, qui contenait la Bulle d'or, portât un frontispice particulier daté de 1612, il nous paraît probable que les deux parties aient été publiées et vendues ensemble, car seul le frontispice de la première partie faisait mention du privilège royal, alors que ce privilège (en date du 24 octobre 1612) était imprimé à la fin de la deuxième partie. Comme nous venons de le dire, les deux parties furent réimprimées en 1633. Le Secq dédia sa traduction française du livre de Canini au cardinal Du Perron, qui était un homme politique influent, en sa qualité de membre du Conseil de régence, et qui professait des idées ultramontaines ${ }^{273}$. Jusqu'en 1608 , il avait représenté la France auprès du Saint-Siège. Force est de constater que non seulement l'auteur du livre (Canini), mais aussi son traducteur (Le Secq) entretenaient des relations privilégiées avec des personnages proches du SaintSiège ou du Collège cardinalice. Le Secq attribue à Canini une très bonne connaissance de l'histoire et de la doctrine de la foi, et estime que le sujet de son livre est nouveau et intéressant, mais aussi difficile et épineux. Il rapporte que Canini lui avait envoyé cet ouvrage ${ }^{274}$ et qu'il décida sur-le-champ de le traduire en français. En ce qui concerne sa méthode de traduction (qui fut également appliquée pour sa version de la Bulle d'or et pour celle de la capitulation de Charles Quint), Le Secq avoue qu'il ne traduisit pas certains noms propres allemands et italiens, puisque leur traduction lui aurait posé de grands problèmes. Par conséquent, il faut estimer que Le Secq connaissait moins bien son sujet que Canini275. Le Secq traduisit plutôt fidèlement son ouvrage. Mais, comme dans chaque traduction, on repère parfois dans sa version de Canini des changements sémantiques ${ }^{276}$. Dans sa traduction de la capitulation impériale, on note que Le Secq traduisit par "ga-

269 Cf. LE SECQ, Histoire, ici la préface »Av Lectevr« (1613), fol. ē-ē iij' .

$270 \mathrm{Cf}$. CioranesCu, Bibliographie de la littérature française du dix-septième siècle, $t$. II, p. 1283.

271 Publiée s.l.n.d.; dix pages in-4\%.

272 Également publiée s.l.n.d.; quatre pages in $-4^{\circ}$. Cette brochure a certainement été rédigée à l'occasion du mariage franco-espagnol entre Louis XIII et l'infante Anne d'Autriche. Le traité scellant leur union fut conclu à Madrid, le 20 août 1612 .

273 Cardinal Jacques Davy Du Perron (1556-1618). Au sujet de ce personnage, voir T. DE MOREMBERT, article »Du Perron (Jacques Davy)«, dans: Dictionnaire de biographie française, vol. XII (1970), col. 339-341.

274 Cf. LE SECQ, Histoire (1613), en particulier l'»Epistre« (six pages non paginées au début du livre, ici fol. ã iij).

275 Cf. ibid., la »Preface«, ici fol. ē iij-ē iij'. Dans les éditions de 1612/1613 et de 1633, la capitulation se trouve p. 31-37 de l'»Abregé«.

276 Cf. BRAUN, Ein italienischer Blick, p. 83. 
belles« le terme italien »gabella«, employé par Canini dans la version italienne. Or, au début du XVII ${ }^{e}$ siècle, en français, la gabelle, initialement appliquée à d'autres formes de l'impôt indirect, avait la seule signification de l'impôt sur le sel, alors que le concept italien signifiait toujours tous les impôts levés sur les vivres, ce qui correspondait effectivement au véritable sens de l'original allemand de la capitulation impériale de Charles Quint ${ }^{277}$.

La réédition de 1633 est presque identique à la première édition de 1612 et 1613. L'éditeur, Robert Bertault, retira seulement la dédicace et le nom du traducteur. Mais il réimprima même le privilège du 24 octobre 1612. Alors que le frontispice faisait mention d'un privilège royal, Bertault n'ajouta à cette édition aucun privilège valable pour sa personne. La réédition comporte également les mêmes erreurs typographiques que la première édition, tandis que l'orthographe des frontispices est différente. Il n'est pas surprenant que ce livre ait été réédité en français en 1633, car les livres français et les éditions françaises des lois fondamentales du Saint-Empire étaient rarissimes avant 1648.

En ce qui concerne les versions françaises intégrales de la Bulle d'or, l'on peut distinguer deux groupes de traduction différents: étant donné la particularité des traductions respectives de l'invocatio Dei, au début de la Bulle d'or, nous qualifions le premier de groupe »individuë«, le deuxième de groupe »indivisible«. La traduction de ce dernier groupe remonte à Heiss ${ }^{278}$. Celui-ci publia pour la première fois sa traduction de la Bulle d'or en 1684; cette édition eut un tel succès que l'on sépara même la traduction de la Bulle d'or du reste de l'ouvrage ${ }^{279}$. La première traduction fut publiée par Abraham de Wicquefort, dans sa monographie sur l'élection impériale, publiée à l'occasion de l'interrègne suivant la mort de Ferdinand III, en 1657, et précédant l'élection de Léopold Ier, en 1658280. D'abord publiée en $1658^{281}$, elle fut rééditée à l'occasion de l'élection de Charles VI, en $1711^{282}$. Le chapitre VII de cet ouvrage contient une version française - la pre-

\section{Cf. Canini, Compendio, p. 17; Le SeCQ, Abregé (1612), p. 34.}

278 HeIss, Histoire de l'Empire (1685), t. III, p. 322-376, ici p. 322: "Au Nom De La Sainte $\&$ indivisible Trinité. Ainsi soit-il $\ll$. La traduction de cette pièce se trouve dans toutes les éditions de l'»Histoire de l'Empire «.

279 HeIss, Histoire de l'Empire (1684), t. II, p. CCXCVIJ-CCCLIV. À la Bibliothèque nationale, on trouve un exemplaire de la Bulle d'or qui a été coupé de l'édition de 1684 de Jean Heiss (BNF: M.10449; exemplaire non identifié avec l'ouvrage de Heiss dans les catalogues). Cet exemplaire contient uniquement les p. CCXCVIJ-CCCLIV du t. II (c'est-à-dire le texte intégral de la Bulle d'or); en haut de la p. CCXCVIJ, on a noté: »10 janvier 1356 \%. 280 Au sujet de son élection et de sa capitulation impériale, cf. BÉRENGER, Léopold Ier, en particulier p. 199-235, avec un résumé des points les plus importants de cette capitulation. La France exerça une influence importante sur sa rédaction. En se fondant sur la relation finale de Battista Nani, qui mettait en relief la mauvaise réputation des Espagnols dans l'Empire et même chez les Autrichiens, Bérenger conclut: "Pour une fois, les intérêts français s'accordaient parfaitement avec ceux des princes allemands et de leurs sujets« (ibid., p. 219). 281 [Abraham DE WICQUEFORT], Discovrs historiqve de l'élection de l'Emperevr et des électevrs de l'Empire. Par le resident de Brandebourg, Paris 1658.

282 Édition de Rouen, 1711 (voir ci-dessus). 
mière version intégrale, autant que nous le sachions - de la Bulle d'or ${ }^{283}$. Le texte du chapitre VII de l'édition de Rouen de 1711 correspond très exactement à la première édition de $1658^{284}$. D'une édition à l' autre, le texte de la traduction française de la Bulle d'or n'a donc pas subi de modifications majeures ${ }^{285}$. Outre la Bulle d'or, l'ouvrage de Wicquefort offre également une version française de la capitulation de l'empereur Ferdinand III (1636)286. Dans l'édition de 1711, c'est également le chapitre XVIII qui contient la capitulation de cet empereur. En général, le texte correspond très exactement au chapitre XVIII de l'édition de 1658, abstraction faite de l'orthographe et de la ponctuation, mais on note aussi d'autres différences mineures ${ }^{287}$.

L'événement qui fournit l'occasion de la publication de la première édition fut, on vient de le dire, l'élection impériale de 1658. En effet, la vacance du trône impérial qui avait incité la diplomatie française à combattre la candidature du Habsbourg, était un événement majeur non seulement pour les Allemands, mais aussi pour les Français. Même si Wicquefort ne publia son livre qu'en 1658, la publication de celui-ci était déjà prévue depuis $1657^{288}$. Finalement, le livre fut donné au public le $1^{\text {er }}$ avril 1658 , à peu près trois mois et demi avant l'élection de Léopold ${ }^{289}$. Dans son épître dédicatoire, Wicquefort évoque cet engouement des Français, qui cherchaient alors à se renseigner par tous les moyens sur l'histoire et le droit public germanique relatif à l'élection de l'empereur. Si ses remarques peuvent être interprétées comme une convention littéraire, la réalité était tout à fait conforme à ses propos, et le livre fut un succès fulgurant, puisqu'il fut encore réédité plusieurs fois au XVIII e siècle, parfois à titre anonyme. Même en 1745, à l'heure de l'élection de Charles VII, son ouvrage fut encore réimprimé. Ainsi, l'histoire confirma l'espoir que certains interlocuteurs avaient donné à Wicquefort, en lui conseillant de ne pas poursuivre son projet d'écrire une histoire générale d'Allemagne, mais de faire un livre sur l'élection de l'empereur, puisque même un livre mauvais ne manquerait pas de connaître un très grand succès auprès du public 290 . Il est vrai également que les Français n'avaient pas alors à leur disposition de bons livres traitant de l'élection impériale. L'ouvrage de Canini tra-

283 Dans l'édition de 1658, p. 59-110.

284 Dans l'édition de 1711, p. 69-129.

285 Textes presque identiques, abstraction faite de l'orthographe et de la ponctuation. Mais, de toute évidence, on a révisé la traduction; par exemple, au chapitre XIX, qui reproduit le formulaire latin de la "procuration «, une omission a été corrigée.

286 Dans l'édition de 1658 , chapitre XVIII (p. 433-475), capitulation de Ferdinand III p. 447-474.

287 Par exemple, une erreur s'est glissée dans l'édition de 1711: à l'article 51, on lit »ayant « au lieu de la leçon correcte »ayent« (la bonne leçon se trouve dans l'édition de 1658, p. 473). 288 En effet, dès le mois d'octobre 1658, le libraire Augustin Courbé eut le privilège d'imprimer et de publier le traité de Wicquefort. Cf., à la fin de l'ouvrage (p. [520]), »Extraict du Priuilege du Roy «; registré par la communauté des libraires le 26 octobre 1657.

${ }^{289}$ Cf. ibid.: »Les Exemplaires ont esté fournis. Acheué d'imprimer pour la premiere fois le 1. Avril 1658«.

$290 \mathrm{Cf}$. la dédicace, ibid., fol. ã ij-ã iij. Pour l'origine de l'ouvrage, cf. aussi l'avertissement de l'éditeur au lecteur, repris dans l'édition de Rouen 1711, fol. ã 2 - [ã 5], ici fol. ã 3' - ã 4. 
duit en français par Le Secq était en réalité le seul véritable livre français sur ce problème. Toutefois, en 1658, Wicquefort note un changement fondamental: un intérêt du public cultivé pour l'élection ${ }^{291}$, qui devait dépasser le seul cercle des hommes politiques et des diplomates, puisque, pour ces personnages, des mémoires manuscrits auraient suffi. Rappelons que, en 1636, à l'occasion de l'élection de Ferdinand III, Richelieu avait fait rédiger un grand nombre de mémoires sur l'élection impériale ${ }^{292}$. Or, en 1658 , ces mémoires dont la connaissance était limitée à un petit groupe de professionnels ne suffisaient plus. Il fallait satisfaire la demande des élites, ce qui pouvait aussi servir à légitimer la politique française. Toujours est-il que la publication de l'ouvrage de Wicquefort fut surtout une entreprise éditoriale et commerciale.

Or, les remarques de Wicquefort permettent aussi une autre interprétation, qui nuance un peu le tableau que nous venons de dresser. En effet, Wicquefort dit clairement que les Français s'intéressaient sinon uniquement, du moins tout particulièrement à l'élection de l'empereur, alors que son projet d'écrire une histoire générale de l'Allemagne ne reçut pas le même encouragement de la part de ses interlocuteurs. Même s'il dépassa lui-même les bornes étroites que son sujet lui avait fixées ${ }^{293}$, cette réserve explique pourquoi l'on devait encore attendre quelques années avant de voir publier en français des ouvrages historiques généraux sur l'Allemagne. Toutefois, il convient de noter que cet auteur choisit délibérément une approche historique de son sujet, alors que d'autres écrivains se limitaient à des prises de position politiques ${ }^{294}$. Outre l'élection en général, Wicquefort attachait aussi une grande importance à chaque électeur en particulier, à sa dynastie et à ses territoires, afin de comprendre les enjeux de cette élection pour chacun.

La première version intégrale de la Bulle d'or, fondement de tout le droit électoral de l'Empire germanique, était donc due à un diplomate étranger. En traduisant cette bulle, Wicquefort préféra la clarté à la beauté du style ${ }^{295}$. Comme on le

291 Cf. [WICQUEFORT], Discovrs historique de l'élection de l'Emperevr et des électevrs de l'Empire, 1658, fol. ã ij'-ã iij: "La France auoit pris si peu de part à celles [sc. les élections] qui se sont faites depuis plus de six vingts ans, que l'on y auoit negligé d'estudier vne matiere, dont la connoissance sembloit estre inutile, \& que l'on n'auoit point eu d'occasion d'apprendre. Mais depuis peu les reuolutions, dont l'Allemagne s'est veuë menacée, apres vn interregne, qui a laissé aux interessez le loisir de faire diuerses negotiations, ont donné aux curieux l'enuie de s'informer de l'estat des affaires de ces quartiers là, \& des particularitez d'vne des plus celebres actions, qui se fassent en l'Europe«.

292 Nous les avons étudiés au premier chapitre.

293 En ce qui concerne le sujet que l'auteur avait abordé, on note qu'il n'évoque pas seulement les questions d'actualité. Plusieurs chapitres abordent le problème de l'origine de l'élection impériale et du collège des sept électeurs, cf., entre autres chapitres, [WICQUEFORT], Discours historique de l'élection de l'Empereur, Rouen 1711, p. 24-39.

294 L'avertissement »Au lecteur « souligne ces deux approches différentes. À ces politiques, l'éditeur oppose le dessein de Wicquefort, qui consistait à écrire en tant qu'historien, selon le vieil idéal de Tacite, cf. ibid., fol. ã 2-[ã 5], ici fol. ã 2'-ã 3'.

295 Cf. dans [ID.], Discovrs historique de l'élection de l'Emperevr et des électevrs de l'Empire, 1658, l'avis »Av Lectevr« (fol. ẽ j-ẽii j', ici fol. ẽ iij), qui évoque, d'un côté, »le tour \& 
verra, la deuxième traduction, qui évinça celle de Wicquefort, fut également l'œuvre d'un résident étranger, plus précisément de Jean Heiss. Malgré le handicap linguistique que les différentes préfaces de ces ouvrages, rédigés par des auteurs étrangers, ne manquaient presque jamais d'évoquer, la France faisait donc régulièrement appel à leur secours pour s'informer du droit public d'Allemagne. Cela montre que ces étrangers qui vivaient en France, en particulier à Paris, constituaient une main-d'œuvre indispensable, car il n'y avait pas de Français qui auraient pu les remplacer. On verra que cette situation changea fondamentalement au XVIII' siècle.

La réédition de 1711 fut très certainement le résultat des préparatifs de l'élection de Charles VI, qui mit de nouveau l'élection impériale au centre de la politique européenne. Au mois de mai 1711, cinq mois avant que le nouvel empereur ne fût élu, le 12 octobre, les autorités françaises non seulement approuvèrent la publication de cette nouvelle édition, mais préconisèrent qu'elle devait être donnée au public sans délai ${ }^{296}$. Le »Discovrs historiqve« sur l'élection impériale fut également publié en annexe de l'»Ambassadeur«, écrit par le même auteur ${ }^{297}$. Toutefois, les premières éditions de l'»Ambassadeur«, publiées en 1680 et $1681^{298}$, puis en $1682^{299}$, année de la mort de l'auteur, après les travaux préparatoires donnés au public depuis 1676, quand Wicquefort était emprisonné en Hollande, ne contenaient pas cet appendice. En revanche, pour la première fois, il fut inséré dans l'édition de 1689. Par la suite, dans l'édition de $1715^{300}$, le »Discours historique « a été reproduit en annexe dans le deuxième volume ${ }^{301}$, tout comme la version

la perfection, qui est ce que l'on aime $\&$ admire aujourd'huy le plus dans vn liure «, et, de l'autre, le fait »qu'il est tres-difficile, de rendre bien Françoises les pieces Allemandes«, telles que la Bulle d'or et les capitulations.

296 Cf. dans [WICQUEFORT], Discours historique de l'élection de l'Empereur, Rouen 1711, l'»Approbation «, au début du livre, à la fin de la table des matières: "J'ay lû par ordre de Monseigneur le Chancelier un Livre intitulé, Discours Historique de l'Election de l'Empereur, \& des Electeurs de l'Empire, par le Sieur Wicfort Résident de Brandebourg. Cet Ouvrage mérite d'être incessamment réimprimé. Fait à Paris le 25. de May 1711. Signé, La Marque-Tilladet «.

297 [Abraham] DE WICQUEFORT, L'Ambassadeur et ses fonctions [...], 2 vol., Cologne [= lieu d'impression fictif] 1689-1690. Le »Discovrs historiqve« (frontispice particulier p. [57], sans mention du lieu ni de la date de publication, texte p. [59]-266 [deuxième pagination]) contient la traduction française de la Bulle d'or (p. 87-109) et les remarques de Wicquefort sur cette loi fondamentale (p. 109-116). On y trouve également la traduction française de la capitulation impériale de Ferdinand III (p. 240-250).

298 ID., L'Ambassadeur et ses fonctions [...], 2 vol., La Haye 1680-1681; ouvrage dédié à Georges-Guillaume, duc de Brunswick-Lunebourg.

299 ID., L'Ambassadeur et ses fonctions [... ], 2 vol., La Haye 1682; ouvrage également dédié à Georges-Guillaume, duc de Brunswick-Lunebourg.

${ }^{300}$ ID., L'Ambassadeur et ses fonctions [...]. Seconde partie, 2 vol., Cologne [lieu de publication fictif] 1715.

${ }^{301}$ ID., Discours historique de l'élection de l'Empereur, et des électeurs de l'Empire. Par le resident de Brandebourg; ibid., t. II, deuxième pagination, p. [67]-325; avec une dédicace à Séguier, duc et pair et chancelier de France. 
française de la Bulle d'or et celle de la capitulation de l'empereur Ferdinand III ${ }^{302}$. En revanche, l'édition de 1724 a été imprimée sans le "Discours historique et sans ces deux lois fondamentales; cette édition a été préparée au moins partiellement par Jean Barbeyrac, traducteur d'un traité latin sur la justice compétente pour juger les ambassadeurs, qui a été inséré dans le deuxième volume ${ }^{303}$. Toutefois, l'auteur de la préface de cette édition mentionne, comme l'un des principaux ouvrages dus à cet écrivain politique, le "Discours historique de l'élection de l'Empereur «, composé en 1657, »à l'occasion de l'élection future de l'Empereur Léopold«, alors que Wicquefort était résident de l'électeur de Brandebourg auprès de la cour de France ${ }^{304}$. Dans la préface, l'éditeur explique l'omission du »Discours historique« par deux raisons: premièrement, depuis la rédaction de ce traité, en 1657, un grand nombre d'ouvrages étaient parus, livres que l'éditeur juge meilleurs et plus exacts que le traité de Wicquefort; deuxièmement, il note que ce traité n'a aucun rapport intrinsèque avec l'»Ambassadeur «, puisque ce dernier livre est consacré à un sujet tout à fait différent ${ }^{305}$. Pourtant, dans l'édition revue et publiée en $1730^{306}$, le »Discours historique « fait partie du deuxième volume ${ }^{307}$; comme dans les éditions précédentes, il comprend la version française de la Bulle d'or, les remarques de Wicquefort sur cette loi fondamentale ainsi que sa traduction de la capitulation de Ferdinand III ${ }^{308}$. Toutes les traductions françaises de la Bulle d'or publiées en annexe à l'»Ambassadeur« de Wicquefort appartiennent au groupe »individuë« 309 . On sait que dès 1682 ce dernier livre de Wicquefort a été traduit en allemand ${ }^{310}$.

Le groupe »indivisible « comprend deux traductions légèrement différentes: la première remonte à Jean Heiss; la deuxième se trouve chez Simon Jacquet. Deux différences dans la traduction du début du préambule de la Bulle d'or permettent

302 Bulle d'or: ibid., p. 101-127; les remarques de l'auteur sur la Bulle d'or: ibid., p. 127 135; la capitulation de Ferdinand III: ibid., p. 290-303.

303 ID., L'Ambassadeur et ses fonctions [...]. Nouvelle édition. Augmentée d'un traité du juge competent des ambassadeurs [...], 2 vol., La Haye 1724. Cette édition contient un "Avertissement《 expliquant l'histoire de l'»Ambassadeur « de Wicquefort et donnant des renseignements biographiques sur l'auteur, qui avait passé plus de trente ans de sa vie à Paris.

304 Ibid., t. I, »Avertissement«, sans mention de l'auteur (non paginé), fol. **-[**4]', ici fol. **.

305 Cf. ibid., fol. [**4].

306 Abraham DE WICQUEFORT, L'Ambassadeur et ses fonctions [...]. Nouvelle édition augmentée [...]. Seconde édition revue $\&$ augmentée dans les notes du traducteur, 2 vol., Amsterdam 1730; collection factice de plusieurs livres de différents auteurs (pagination multiple). ${ }^{307}$ Discours historique de l'élection de l'Empereur, et des électeurs de l'Empire. Par le résident de Brandebourg; ibid., t. II, p. [57]-267 (deuxième pagination); la dédicace est signée »D.W.R.D.B.« (p. [60]).

308 Bulle d'or: ibid., p. 87-109; remarques de l'auteur sur la Bulle d'or: ibid., p. 109-115; capitulation de Ferdinand III: ibid., p. 240-250.

309 Nous employons cette orthographe, puisqu'elle est majoritaire, mais on trouve aussi l'orthographe »individue «.

$310 \mathrm{Cf}$. le chapitre IX. 
de les qualifier respectivement de traduction »chose «/»chandeliers « 311 et "présente «/»flambeaux « ${ }^{312}$. En 1711, la Bulle d'or fut publiée dans le sixième volume des »Voyages historiques de l'Europe « ${ }^{313}$. Le quinzième et dernier chapitre est entièrement consacré à la Bulle d'or et contient une version française de cette loi fondamentale, divisée en trente chapitres ${ }^{314}$. Cette édition reproduit le texte de la version »indivisible « ${ }^{315}$. La traduction n'est pas annotée. L'auteur donne une brève introduction à l'histoire de la Bulle d'or, son importance dans la Constitution du Saint-Empire et les originaux de ce document ${ }^{316}$. Cette introduction fut presque littéralement recopiée, en 1741, dans l'édition de la Bulle d'or publiée par l'imprimeur-libraire André Chevalier à Luxembourg ${ }^{317}$. Cet auteur adhère à la théorie du gouvernement mixte. Il considère que des éléments démocratiques, aristocratiques et monarchiques sont mêlés dans la Constitution du Saint-Empire en général ${ }^{318}$, avec une prédominance des états de l'Empire ${ }^{319}$, excluant formellement les hypothèses selon lesquelles il s'agirait d'une démocratie, d'une aristocratie ou d'une monarchie (absolue ou limitée) ${ }^{320}$.

L'année 1711 fut une année faste pour la publication de la Bulle d'or. C'était l'année de l'élection de Charles VI, un événement avec des conséquences sur

311 Heiss, Histoire de l'Empire (1685), t. III, p. 322: »Charle[s] Par la Grace de Dieu, Empereur des Romains toûjours Auguste \& Roi de Boheme, à la memoire perpetuelle de la chose [...] il a ôté leurs chandeliers du lieu où ils étoient, afin qu'ils soient aveugles $\&$ conducteurs d'aveugles $\ll$.

312 JACQUET, Recueil precieux, t. I, p. 1-16 et 257-317, ici p. 5-6: „Charles, Par la grace de Dieu, Empereur des Romains, toujours Auguste \& Roi de Bohëme, en perpetuelle mémoire de la présente [...] il a ôté leurs flambeaux du lieu où ils étoient, afin qu'ils soient aveugles \& conducteurs d'aveugles «. Cf. aussi ID., Droit public d'Allemagne, t. IV, p. 246-317.

${ }^{313}$ La Bulle d'Or, les ceremonies de l'élection et du couronnement de l'Empereur; l'origine, les droits, les prerogatives des électeurs, princes \& états de l'Empire. Et ce qu'il y a de plus curieux en Allemagne. Le tout contenu dans le sixiéme volume des Voyages historiques de l'Europe, Paris 1711. Avec une carte des dix cercles de l'Empire. Le privilège imprimé au début du volume (après l'»Avis« au lecteur et la table des chapitres, mais avant la carte) date du 10 mai 1711 et fut registré le 15 du même mois.

314 Ibid., p. 227-383.

315 Sous-groupe »chose»/»chandeliers«.

316 La Bulle d'Or, 1711, p. 221-226.

317 Cf. La Bulle d'Or, suivie de la sanction pragmatique \& loi perpétuelle. Suivant la copie imprimée à Amsterdam, Luxembourg 1741, p. 3-5. Avec un seul changement notable par rapport au texte de 1711: la mention de la mort de l'empereur Charles VI, qui a donné lieu à la publication de 1741. Dans cette dernière édition, la traduction de la Bulle d'or se trouve p. 5-59.

318 Dans les États territoriaux, il existe des formes pures de monarchie (princes ecclésiastiques et séculiers), d'aristocratie et de démocratie (villes gouvernées par les nobles ou par le peuple); La Bulle d'Or, 1711, p. 65-66.

319 „En un mot; [l'empereur] c'est un Prince sur le Trône Imperial; mais ce sont les Etats de l'Empire qui y donnent la Loy. Il faut donc conclure que ce Gouvernement est meslé de Démocratie, puisque les Peuples y sont appellez; d'Aristocratie, à cause que les Electeurs \& les Princes y tiennent la principale autorité; \& de Monarchie, parce que l'Empereur revêtu des ornemens de la Royauté, preside aux Etats de l'Empire, qui ne peuvent rien faire sans luy, comme il ne peut rien faire sans eux «, ibid., p. 67-68.

320 Ibid., p. 66-67. 
toute l'Europe en pleine guerre de la Succession d'Espagne. Cela explique sans doute l'engouement des lecteurs français pour cette loi fondamentale. Du mois de mai au mois de juillet 1711, l'éditeur parisien Jollet fit imprimer le texte de la Bulle d'or en français, sans préface, sans commentaire ni annotation ${ }^{321}$. De toute évidence, il s'agit d'une publication de circonstance, destinée à satisfaire le plus rapidement possible le désir du public de connaître le texte de cette loi réglant l'élection du successeur de l'empereur Joseph Ier, décédé le 17 avril 1711. Les différentes datations laissent supposer que l'ouvrage ait été distribué en trois livraisons contenant respectivement les articles I à IX, X à XXI et XXII à XXX de la Bulle d'or, avec, toutefois, une pagination continue. Cette manière de publication serait une preuve de la forte demande du public de se voir renseigné très rapidement sur le texte de la Bulle d'or, trop rapidement pour que les ateliers de l'imprimeur puissent y satisfaire d'un seul coup. Ce livre reproduit la traduction du groupe »indivisible «322. La même année parut à Toulouse une autre édition de la Bulle d'or, qui elle est incomplète, mais contient en revanche des explications sur les prérogatives des empereurs et des électeurs. Il semble bien que, comme l'édition parisienne, cette publication toulousaine de la Bulle d'or en langue française devait initialement comporter trois livraisons. En effet, les deux premières livraisons reproduisent les articles I à IX et Xà XXI de la Bulle d'or. Toutefois, la troisième livraison fait défaut. Cette édition comporte d'ailleurs une autre indication précieuse: sur le frontispice, l'éditeur a fait imprimer le prix de l'ouvrage. C'est ainsi que nous savons que la première livraison, y compris les explications sur les prérogatives des empereurs et des électeurs, coûta quatre sous, et la deuxième, deux sous ${ }^{323}$. La première livraison comprend quarante-neuf pages; la deuxième, vingt-deux pages et l'adresse de l'éditeur ${ }^{324}$. Dans la première livraison, un peu plus de dix-sept pages sont consacrées aux électeurs ${ }^{325}$. En les déduisant du nombre intégral des pages, on arrive à un prix de deux à trois sous pour la première livraison sans ces explications sur les électeurs. En ajoutant deux sous pour les neuf derniers chapitres de la Bulle d'or, qui manquent dans l'édition de Toulouse, on arrive à un prix de six à sept sous, en 1711, pour le texte intégral de la Bulle d'or en français, sans commentaire ni documents supplémentaires, dans une édition en

321 La Bulle d'Or, Paris 1711 (publiée chez Jollet). La date de »May 1711 « se trouve imprimée en bas de plusieurs pages et semble bien correspondre à la date de l'impression, cf. ibid.,

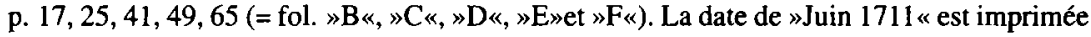

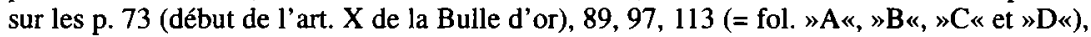
celle de "Juillet 1771 «p. 121 (début de l'art. XXII de la Bulle d'or), 137, 145, 161 (= fol. $» A \ll, » B \ll, » C \ll$ et $» D \ll)$.

322 Sous-groupe »chose»/»chandeliers«, ibid., p. 1-164.

${ }^{323}$ La Bulle d'Or, contenant ce qui doit s'observer à l'élection des Empereurs. Avec quelques autres pieces curieuses sur le même sujet. Et une instruction concernant la dignité \& les prérogatives des électeurs de l'Empire [...], Toulouse 1711; deuxième frontispice: Suite de la Bulle d'Or [...], ibid. 1711 .

324 Le frontispice est toujours compris dans ces paginations, ibid., p. [1] et [1 bis].

325 Ibid., p. 32-49 (première pagination). 
petits caractères 326 . Comme dans l'édition parisienne, ce livre reproduit la traduction du groupe »indivisible «327. On constate que, très certainement, l'éditeur ne connaissait pas bien la Bulle d'or, puisque, à la fin de la première livraison, c'està-dire à la fin de l'article IX, il note: »Fin de la Bulle d'Or «328. D'une préface que Loyau a ajoutée au début de la deuxième partie il résulte qu'il n'avait pris connaissance de la suite de la Bulle d'or qu'après la publication de la première livraison. Il est probable que les différentes livraisons publiées à Paris aient été envoyées en province, et que là on en ait pris connaissance au fur et à mesure que l'édition parisienne avança ${ }^{329}$.

En réalité, le traité sur les prérogatives des empereurs et des électeurs est l'ouvrage de l'abbé Louis de Courcillon de Dangeau (1643-1723), membre de l'Académie française et auteur d'une »Nouvelle metode de geografie historique«. En effet, un ouvrage portant exactement le même titre que celui mentionné dans la préface du traité des électeurs, dans l'édition toulousaine de la Bulle d'or et dans l'édition indépendante parisienne, parut d'abord à Paris, chez A. Lambin, en 1697, puis, également à Paris, chez D. Jollet, en $1706^{330}$. La partie de la »Nouvelle metode« que cet auteur donna au public en 1697 contient seulement les cartes du monde, de l'Europe et de la France. Comme l'auteur le précise dans les éditions de son traité »Des électeurs « ${ }^{331}$, la partie concernant l'Allemagne n'avait pas encore été publiée au moment où il décida de publier séparément la carte des électorats de l'Empire. Dangeau fit aussi publier à part des descriptions de son ouvrage volumineux ${ }^{332}$.

326 En déduisant toutes les pièces liminaires, les deux premières livraisons occupent cinquante pages dans l'édition toulousaine et cent vingt dans celle de Paris.

327 Sous-groupe $» c h o s e ~ « / » c h a n d e l i e r s \ll$, ibid., p. 3-32 (première pagination) et p. 3-22 (deuxième pagination).

328 Ibid., p. 32.

${ }^{329}$ Cf. ibid., p. [2] (deuxième pagination): "Depuis l'Impression de la Bulle D'Or qu'on a donné au Public, on a reçû cette Suite; comme elle contient des Articles très-curieux, \& sans lesquels on ne peut avoir une pleine connoissance de ce qui regarde l'Election de l'Empereur, on a crî faire plaisir de la donner aussi au Public, d'autant mieux, que sans cela cette Piece seroit imparfaite «.

330 [Louis DE COURCILLON DE DANGEAU], Nouvelle metode de geografie historique pour aprendre facilement, \& retenir long-tems la geografie moderne \& l'anciène [...], Paris 1697. Malgré la date de publication de l'ouvrage, certaines cartes sont datées de 1699. Ce volume contient exclusivement des cartes du monde, de l'Europe et du royaume de France. [ID.], Nouvelle metode de geografie historique [...], Paris 1706. Cet ouvrage contient quelques remarques sommaires sur les électeurs du Saint-Empire dans la description d'une carte du royaume de France et des pays limitrophes (p. 7-8).

331 [ID.], Des électeurs, s.l. 1695 (tract, 1 p. in-folio); [ID.], Des électeurs, Paris s.d. (14 p. in$8^{\circ}$ ).

332 Pour ces pièces, qui sont de toute évidence destinées à faire connaître l'ouvrage (une publicité avant la naissance du public moderne), nous ne connaissons qu'un seul exemplaire de chacune de ces deux éditions conservées dans le cabinet des manuscrits de la Bibliothèque nationale, comme documents insérés dans un volume de mélange de documents manuscrits et imprimés: [ID.], Nouvèle metode de geografie historique [...], Paris 1697 (BNF, Coll. Clairambault 492 fol. 73-90). En fait, c'est un tiré à part de l'avertissement au lecteur tiré de l'ouvrage de géographie historique du même auteur en plusieurs parties in-folio avec des no- 
Outre les rééditions de la Bulle d'or, l'année 1711 provoqua également de nouvelles éditions de l'ouvrage de Wicquefort sur l'élection de l'empereur. Or, il est intéressant de voir qu'un ouvrage qualifié généralement de réédition de sa monographie sur l'élection impériale, mais qui n'est en réalité qu'une anthologie de certains chapitres, parue à titre anonyme et sans privilège ${ }^{333}$, n'a pas puisé sa traduction de la Bulle d'or dans ce livre de Wicquefort; en effet, cette traduction appartient au groupe »indivisible « ${ }^{334}$. L'éditeur de cette nouvelle édition abrégée a omis la brève introduction que Wicquefort avait ajoutée au début de sa version française de la Bulle d'or ${ }^{335}$. Il est bizarre que tout en rééditant une partie de l'ouvrage de Wicquefort l'éditeur ait choisi d'y ajouter une traduction de la Bulle d'or différente ${ }^{336}$; étant donné qu'il ne semble pas avoir apporté un très grand soin à cette édition, qui paraît avoir été établie très rapidement pour profiter de l'occasion de l'élection de Charles VI et la débiter sans délai, il est improbable que la supériorité de la traduction du groupe »indivisible« ait guidé son choix. En revanche, on peut supposer qu'il ait délibérément puisé cette traduction dans un autre ouvrage afin de cacher l'origine du texte, puisque, du moins en France, un autre éditeur disposait, en 1711, du privilège exclusif de rééditer la monographie de Wicquefort. Cette hypothèse est confortée par le fait que l'éditeur de l'édition publiée sine loco, en 1711, a retiré toute allusion à l'auteur de l'ouvrage du frontispice et du texte même, et qu'il y a apporté certaines modifications propres à brouiller les pistes. En effet, nous savons par d'autres indices que Wicquefort était bien souvent l'objet de contrefaçons qui contribuèrent même à la ruine de Steuker, c'est-à-dire du premier libraire par lequel il avait fait publier l'»Ambassadeur «337. Mais, malgré ces modifications et malgré le choix d'une version de la Bulle d'or différente, il est clair que l'ouvrage publié anonymement en 1711 est l'œuvre de Wicquefort ${ }^{338}$. C'est chez lui, et pas directement »dans les sources les plus pures

tes manuscrites; [ID.], Nouvelle metode de geografie historique [...], Paris 1706 (BNF, Coll. Clairambault 492 fol. 91-177'). Pièce contenant le privilège, l'avertissement et la description des cartes et des pièces qui composent l'ouvrage du même auteur.

333 [Abraham DE WICQUEFORT], Discours historique de l'élection de l'Empereur, et de l'origine des électeurs de l'Empire, avec la Bulle d'Or de Charles IV. et la capitulation de Joseph I. Empereur dernier mort, [s.1.] 1711 [sans privilège].

334 Sous-groupe »chose«/»chandeliers «.

335 Voir ci-dessus.

336 Outre la traduction de la Bulle d'or, cette édition anonyme contient la version française de la capitulation impériale de Joseph Ier. Cf. [WICQUEFORT], Discours historique de l'élection, [s.l.] 1711, p. 122-184 et p. 203-265.

337 Cf. WICQUEForT, L'Ambassadeur, édition de 1724, préface de l'éditeur (non paginée), ici fol. ${ }^{* *} 3^{*}$ et fol. [**4]'. L'auteur de la préface souligne, en particulier, que les éditions datées de 1690 à Cologne constituent des contrefaçons.

338 En effet, l'édition de [WICQueforT], Discours historique de l'élection, [s.l.] 1711 est constituée d'un »Avis« au lecteur, fol. $)(2-)(2$ ', qui a remplacé celui de 1658 , de neuf chapitres du traité de Wicquefort, de la Bulle d'or, de remarques sur cette bulle et de la capitulation de Joseph Ier, avec des remarques à son égard. Les chapitres I à VI correspondent aux six premiers chapitres du traité de Wicquefort. Le chapitre VII correspond au chapitre XVIII de Wicquefort; le chapitre VIII, au chapitre XIX de Wicquefort, mais au lieu du titre initial (»De l'Election de l'Empereur «), il s'intitule: »De l'Election de l'Empereur, avec une Rela- 
du droit public d'Allemagne « que l'éditeur a puisé ce travail ${ }^{339}$, pour tirer profit de cette conjoncture très favorable à une telle entreprise éditoriale ${ }^{340}$.

En revanche, pour une autre édition anonyme et posthume du traité de Wicquefort, parue en 1741, après la mort de Charles VI ${ }^{341}$, on peut noter que l'éditeur choisit délibérément la traduction de Heiss au lieu de celle du résident de Brandebourg. Dans son »Avis« au lecteur, il souligne la plus grande clarté de la version de Heiss qui l'a incité à insérer ce texte à la place de la traduction plus obscure de Wicquefort. Le même éditeur remplaça la capitulation de Ferdinand III que Wicquefort avait publiée en 1658 par celle de Charles VI à cause des modifications que les capitulations impériales avaient subies entre-temps, notamment en ce qui concerne les règlements plus précis relatifs à la procédure de la mise au ban d'un prince de l'Empire. Dans son »Avis«, l'éditeur, dont l'identité nous échappe, évoque l'engouement du public européen pour les publications concernant l'élection impériale au lendemain du décès de Charles VI. Il ne veut pas cacher que le traité qu'il publie est dû à la plume d'un autre auteur, dont, cependant, il ne révèle pas l'identité. Cet ouvrage est donc classé parmi les livres anonymes à la Bibliothèque nationale; notre attribution à Wicquefort se fonde sur la comparaison avec son »Discovrs historique « de 1658. Par ailleurs, au moment de l'élection impériale suivante, en 1745, cet ouvrage fut réédité sans mention de l'auteur, avec le même titre, également à Amsterdam, comme l'édition précédente, et vendu par un libraire parisien, David ${ }^{342}$. Abstraction faite d'une accentuation différente sur le frontispice, les deux éditions sont fort semblables; on constate que même une erreur évidente dans une note de bas de page n'a pas été corrigée ${ }^{343}$. Mais les deux éditions ne sont pas tout à fait identiques ${ }^{344}$. Toutefois, l'édition de 1745 repro-

tion des ceremonies qui se sont faites à l'Election de Ferdinand III«. Le chapitre IX correspond au chapitre XX de Wicquefort. La capitulation de Joseph $\mathrm{I}^{\text {er }}$ et les remarques à ce sujet ne se trouvent pas dans le traité de Wicquefort, mais elles correspondent en partie littéralement aux remarques que Wicquefort a faites sur la capitulation de Ferdinand III.

339 Pour la citation, cf. ibid., "Avis «, fol. $)(2-)(2$ ', ici fol. $)(2$.

340 C'est la part de vérité dans les explications de l'éditeur: »L'Interregne present dans l'Empire d'Allemagne, \& l'Election prochaine de l'Empereur, fait l'entretien de bien des gens; chacun prend plaisir d'en raisonner selon sa portée, mais tous ne rencontrent pas juste, particuliérement ceux qui n'ont aucune teinture des affaires de ce pays là: c'est pour les édifier que l'on a entrepris de donner au public ce discours « (ibid.).

341 [Abraham DE WICQUEFORT], Traité historique de l'élection de l'Empereur, avec les ceremonies qui s'y observent; la Bulle d'Or, et tout ce qui concerne les fonctions \& prérogatives des électeurs, 2 t. en 1 vol., Amsterdam (édition vendue à Paris, chez Le Gras) 1741. Un autre tirage de cette édition a été mis sur le marché la même année avec la mention suivante: Et se vend à Paris, chez David fils; 2 vol.

342 Il n'est toutefois pas certain que ce libraire, David l'aîné, et celui qui avait vendu une partie de l'édition de 1741, David fils, soient la même personne. Cf. [Abraham DE WICQUEFORT], Traité historique de l'élection de l'Empereur, avec les cérémonies qui s'y observent; la Bulle d'Or, et tout ce qui concerne les fonctions \& prérogatives des électeurs, 2 vol., Amsterdam (édition vendue à Paris, chez David l'aîné) 1745 .

343 Ibid., t. I, p. 120, n. 1 (éditions de 1741 et de 1745).

344 Par exemple, dans la préface, t. I, p. IV, on note deux petites différences: »leurs préémi- 
duit, tout comme celle de 1741, la traduction de la Bulle d'or de Heiss ${ }^{345}$ et une traduction de la capitulation impériale de Charles VI ${ }^{346}$.

Les nombreuses traductions de la Bulle d'or que nous avons signalées montrent l'attention particulière que les savants du XVII et du XVIII ${ }^{e}$ siècle ont prêtée à cette loi fondamentale du Saint-Empire. Ses éditions françaises servaient non seulement aux personnes curieuses de connaitre le droit public allemand, mais aussi aux auteurs qui s'intéressaient à d'autres sujets de l'histoire politique et du droit des gens. Jean Rousset eut par exemple recours à la Bulle d'or dans son ouvrage sur l'ordre de préséance entre les princes de l'Europe et leurs ambassadeurs. En effet, dans les deux derniers chapitres de ce livre, il fournit un »Raisonnement Général« sur le rang des électeurs, des princes et des autres membres de l'Empire ainsi qu'un »Reglement touchant la Séance \& le Rang des Electeurs «, qui se fondent tant sur une analyse de la Bulle d'or que sur l'étude des juristes et des historiens qui ont traité ces problèmes ${ }^{347}$. Malgré cela, on note que ce sont surtout les élections impériales qui représentent des périodes fastes pour l'édition de la Bulle d'or, ce qui n'est pas étonnant, puisque cette loi fondamentale réglait l'élection du roi des Romains ou de l'empereur. Même à la fin de la période étudiée, de toute évidence, il y avait encore une demande de la part du public francophone d'avoir à sa disposition de nouvelles éditions du texte de la Bulle d'or en français, alors qu'une explication juridique détaillée de cette loi, comme en donnaient de nombreux ouvrages de droit public, n'était pas toujours jugée indispensable. C'est pourquoi on voit apparaître, par exemple en 1741, une édition du texte français, sans commentaire. Dans le contexte du manque d'un héritier mâle à Charles VI, il n'est pas surprenant que cette édition (en fait, il s'agit de deux éditions presque identiques, mais distinguées par une vignette différente ornant le frontispice) soit accompagnée d'une traduction française de la Pragmatique Sanction promulguée par cet empereur afin de régler la succession dans la maison d'Autriche ${ }^{348}$. Dans cette publication de la Bulle d'or, de toute évidence due à un éditeur favorable aux Habsbourg, on se limite à une brève introduction expliquant l'importance de cette loi fondamentale, son histoire, les originaux conservés et le cérémonial qui y est fixé $e^{349}$, sans ajouter d'autre commentaire. Sur le premier point, cette introduction va certainement trop loin, en prétendant que la Bulle d'or était le fondement des droits de tous les membres de l'Empire. De cette manière, elle occulte le sens ori-

nences« au lieu de »leur prééminence « et »a paru« au lieu de »a parue«. En revanche, la forme étrange du nom de Jean Heiss (»Heisse«) a été conservée.

345 Ibid., t. I, p. [81]-147.

346 Ibid., t. II, p. 259-342 et le »revers»de Charles VI p. 342-345.

347 [Jean] RousSET, Mémoires sur le rang et la préséance entre les souverains de l'Europe et entre leurs ministres réprésentans suivant leurs différens caractères [...]. Pour servir de supplement à l'Ambassadeur et ses fonctions de Mr. de Wicquefort, Amsterdam 1746, ici p. 184-203 et 204-237. Dans le premier chapitre, l'auteur fournit une liste très précise de l'ordre observé entre les différents états de l'Empire, en allant des querelles de préséance entre les électeurs jusqu'à l'ordre de séance des villes impériales.

${ }^{348}$ La Bulle d'Or, suivie de la sanction pragmatique (1741). Le texte de la Pragmatique Sanction a un frontispice particulier, malgré la pagination continue; p. [61].

349 Ibid., p. 3-5. 
ginal de la loi, qui concernait surtout les princes électeurs. D'ailleurs, l'introduction évoque explicitement la vacance du trône impérial, après la mort de Charles VI, qui devait inciter les lecteurs à se pencher sur ce document.

La traduction qui s'imposa en France est celle de Jean Heiss. Il est vrai qu'avant lui, autant que nous sachions, personne n'avait pris la peine de publier une traduction française intégrale de la Bulle d'or, à l'exception d'Abraham de Wicquefort. Or, sous l'Ancien Régime, la traduction de ce dernier fut déjà jugée moins claire, parfois même accusée d'être plus obscure que celle de Heiss. Sur le fond, les successeurs de Heiss ne modifièrent pas sa traduction de la Bulle d'or, mais ils augmentèrent le nombre de notes expliquant le texte; parfois, ces notes signalent des difficultés d'interprétation que même les juristes allemands avaient du mal à résoudre ${ }^{350}$. Le préambule de la Bulle d'or, dont l'original est entièrement rédigé en latin, met l'empereur et les électeurs en garde contre les sept péchés mortels, et appelle ces derniers à l'union. Il fait l'éloge des princes électeurs du Saint-Empire, en des termes qui sont devenus célèbres. Ces expressions ont servi non seulement à mettre en relief le rôle constitutionnel primordial des électeurs comme »colonnes« sur lesquelles reposait l'Empire, à l'époque moderne, mais aussi à caractériser leur fonction dans le titre de la dernière grande monographie qui leur a été consacrée à ce jour ${ }^{351}$.

\section{Les versions françaises des capitulations impériales}

Sous l'Ancien Régime, les auteurs français prêtèrent une attention toute particulière aux capitulations impériales, les tenant pour les lois fondamentales les plus importantes de l'Empire à côté de la Bulle d'or et de la paix de Westphalie ${ }^{352}$. Si,

$350 \mathrm{Cf}$., par exemple, HeIss, Histoire de l'Empire, 1733 (édition in- $8^{\circ}$ ), t. VI, note à propos du chapitre XVI de la Bulle d'or, concernant les »Pfahlbürger «: »Les Jurisconsultes Allemands ne sont pas d'accord entr'eux du véritable sens de cet article« (p. 424, n. a).

351 Axel GotTHARD, Säulen des Reiches. Die Kurfürsten im frühneuzeitlichen Reichsverband, 2 vol., Husum 1999 (Historische Studien, 457/1-2).

352 Les mémoires français sur les capitulations impériales sont très nombreux; en France, on trouve également des mémoires latins sur ce sujet, par exemple un mémoire bilingue (latin/ allemand sur deux colonnes) concernant la capitulation de Charles VI, cf. Ass. nat. 240: »Capitulatio Caroli VI Imperatoris« (titre, fol. 2; mémoire de 134 folios). Pour un commentaire français de la capitulation de François Ier, rédigé probablement au dépôt des archives des Affaires étrangères, cf. AE, MD All. 82 fol. 161-185': »Remarques sur les articles les plus intéressans de la capitulation de l'Empereur François Ier, mars 1755«, sur deux colonnes: résumé des articles de la capitulation à gauche, commentaire à droite; deuxième copie: $\mathrm{AE}$, MD All. 102 fol. 5-22'. Au dépôt des archives, on copiait aussi le texte des capitulations impériales; en règle générale, il s'agit d'extraits; cf., par exemple, l'»Extrait de l'article huit de la capitulation de l'Empereur Charles VII. Paragraphe trente et unième « (AE, MD All. 65 fol. 304-305); l'»Extrait de la capitulation impériale de l'Empereur Charles VI du 12 octobre 1711 et ratifié le 19e décembre de la mesme année «(AE, MD Autriche 2, fol. 234-244'), copie s.d. Parfois, on recopiait le texte intégral d'une capitulation; cf. la version française de celle de Joseph II (1764) dans AE, MD Autriche 38 fol. 74 et fol. 75-166. Pour des extraits des capitulations impériales conservées dans d'autres fonds, cf., par exemple, bibliothèque 
en droit public français, il n'y avait pas de type d'acte public comparable soumettant l'exercice du pouvoir souverain à certaines conditions négociées avant l'avènement du nouveau monarque, il en existait non seulement dans l'Empire, mais aussi dans d'autres pays d'Europe centrale, en particulier dans les monarchies électives et dans les royaumes avec une représentation étatique très influente ${ }^{353}$. La capitulation confirmait, voire augmentait les droits et les prérogatives des ordres, en particulier des électeurs. "Rédigée avant chaque élection, la capitulation impériale était la meilleure protection des libertés germaniques«, écrit Jean Bérenger ${ }^{354}$. Or, il y avait un décalage important entre la teneur des capitulations et la politique des empereurs. Certains d'entre eux prenaient une très grande liberté dans l'application des capitulations qu'ils avaient jurées à leur élection. Cependant, l'on constate que le respect que les empereurs accordèrent à leurs capitulations dans la deuxième moitié du XVIIe siècle et surtout dans la première moitié du XVIII diffère sensiblement du mépris avec lequel Charles Quint avait traité la sienne. Les Français étaient souvent conscients de cette différence entre norme juridique et réalité politique, une différence, voire une opposition, qui se dégageait particulièrement au travers du respect ou du non-respect des capitulations impériales ${ }^{355}$.

Initialement, Heiss avait publié uniquement la capitulation de Léopold Ier, qui avait été la dernière capitulation jurée par un empereur avant la publication de la première édition de son ouvrage. Même dans la troisième édition, parue en 1694, c'est-à-dire quatre ans après la rédaction de la capitulation de Joseph Ier, qui date de 1690 , Heiss continua à donner aux lecteurs uniquement la traduction de la capitulation de Léopold Ier (1658). En effet, en 1694, Léopold régnait toujours, alors qu'on ne savait pas encore si son successeur désigné, le roi des Romains Joseph, accéderait jamais à la tête de l'Empire. D'ailleurs, le récit chronologique de son »Histoire de l'Empire « finissait avec la paix de Westphalie, conclue sous Ferdinand III. En 1711, son premier continuateur, Bourgeois du Chastenet, y ajouta la

de l'Arsenal, ms. 4746 (fonds d'Espagnac), fol. 13-15': „Extrait de la capitulation impériale du 18e juillet 1658« (ibid., fol. 1-3': "Extrait de la Bulle d'or «; fol. 129-130: extraits du traité de Passau et de la paix de religion d'Augsbourg, etc.); Lille, bibliothèque municipale, fonds Godefroy, ms. 123, p. 209-211, 212-217: »Extraict de la capitulation impérialle et promesse faite aux électeurs par l'Empereur Ferdinand second, en l'an mil six cens dix-neuf, après son élection« [par Théodore Godefroy].

353 Pour la Hongrie, cf. Jean BÉRENGER, Tolérance ou paix de religion en Europe centrale? (1415-1792), Paris 2000 (Bibliothèque d'histoire moderne et contemporaine, 3), p. 272, n. 2: »Il est, évidemment tentant de comparer les anciens coutumes et termes parlementaires de Hongrie à ceux du Saint-Empire«, en particulier la »capitulation électorale« des empereurs qui, dans une certaine mesure, correspondait au diplôme de couronnement des rois de Hongrie.

354 ID., Léopold I Ier, p. 218.

355 Plusieurs auteurs soulignent cette divergence. Cf., par exemple, le "Memoire contenant les constitutions de l'Empire « (1705), p. 14 et p. 27-28, qui définit la capitulation comme "une espece de Contrat que les Electeurs font avec l'Empereur aprés l'avoir élû, \& avant que de consentir qu'il soit couronné «, contrat à l'observation duquel l'empereur s'oblige par serment. 
capitulation de Joseph I ${ }^{\mathrm{er}}$, qui était monté sur le trône après le décès de Léopold, en 1705. Au lieu de remplacer la capitulation de Léopold, Bourgeois du Chastenet établit une édition synoptique des deux capitulations. En mettant en caractères italiques les différences entre les deux textes, il permettait aux lecteurs de comprendre facilement leurs divergences (additions, changements et omissions). Alors que Heiss avait terminé son récit chronologique en 1648, Bourgeois du Chastenet estima qu'il fallait le continuer jusqu'à son époque, tout en respectant le style et surtout la brièveté de l'ouvrage de son prédécesseur. Il mit également à jour la description des états de l'Empire et les généalogies des maisons princières allemandes ${ }^{356}$. Pour des raisons qu'il explique très brièvement dans l'introduction de sa nouvelle édition de l'»Histoire de l'Empire«, cet auteur augmenta non seulement le nombre des documents publiés en annexe, mais ajouta aussi un commentaire plus dense à ceux qui s'y trouvaient déjàa ${ }^{357}$. Vogel, pour sa part, reprit cette traduction synoptique des capitulations de Léopold et de Joseph et, après ces deux documents, ajouta encore celle de Charles VI (1711) ${ }^{358}$. Le fait que, en 1731, Vogel ait publié les traductions françaises de trois capitulations impériales atteste l'importance qu'il accordait à ces documents.

Or, les capitulations subirent des changements importants au cours des Temps modernes. C'est la raison pour laquelle elles ont eu l'histoire la plus compliquée de toutes les lois fondamentales qui aient été traduites en français. Il faut dire qu'il s'agit d'un corpus toujours augmenté, à chaque avènement. L'histoire de leur traduction en langue française est rythmée par les élections qui se sont déroulées dans l'Empire: l'élection difficile de Léopold Ier en 1658, élu pendant l'interrègne, non vivente Imperatore, a donné lieu à la traduction de Wicquefort. Cela est aussi vrai pour les élections du XVIII siècle, qui ont toujours donné lieu à la publication des capitulations. Toutefois, l'»Histoire de l'Empire « de Heiss constitue une exception à cette règle, puisque cet ouvrage était conçu pour donner au public toutes les lois fondamentales de l'Empire (sauf la Paix publique de 1495), sans répondre à une demande précise de la part des lecteurs concernant les capitulations. On note surtout une augmentation importante du nombre des publications depuis

356 Cf. HeIss, Histoire de l'Empire (1711), t. I, »Avis« sur la nouvelle édition, fol. a iij-a iij', ici fol. a iij. Et de souligner la continuité par rapport au style et à la méthode narrative. Pour les différentes capitulations, cf. les éditions de 1684, t. II, p. CCIX-CCLIJ; 1685, t. III, p. 226-271; 1694, t. III, p. 226-271; 1711, t. V, p. 373-481.

357 Cf. Heiss, Histoire de l'Empire (1711), t. I, fol. a iij'. À la fin de cette préface, les notes qui furent ajoutées aux lois de l'Empire ainsi que tous les autres changements faits par rapport aux éditions antérieures de cet ouvrage étaient explicitement attribués à Bourgeois du Chastenet, plénipotentiaire subdélégué du duc et de la duchesse d'Orléans aux conférences de Francfort concernant les affaires palatines.

358 En 1715, l'éditeur hollandais Guillaume de Voys avait déjà publié cette capitulation parmi les »Pieces ajoutées à cette Edition de Hollande«, cf. HEISs, Histoire de l'Empire, édition de 1715, t. IV, p. *3' (table des matières) et p. 448-514 (capitulation de Charles VI). Vogel ne connaissait peut-être pas cette édition publiée à La Haye. Cf. également ibid.,

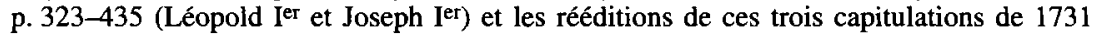
(in- $8^{\circ}$ ), t. VIII, p. 97-293; 1731 (in-4o), t. III[/2], p. 186-244; 1733 (in-8 ${ }^{\circ}$ ), t. VIII, p. 15273; 1733 (in-4), t. II, p. 589-673. 
1740; cela vaut aussi pour la Bulle d'or. L'explication est évidente: l'interrègne qui suivit la mort de Charles VI, la perte de la dignité impériale que subit provisoirement la maison de Habsbourg, l'élection difficile de Charles VII, puis celle de François Ier constituèrent autant d'éléments pour soutenir l'intérêt du public francophone concernant les événements dans l'Empire. En outre, les auteurs justifient leurs publications en soulignant que les capitulations sont devenues les lois fondamentales les plus importantes, puisque plus récentes que la paix de Westphalie et les autres lois fondamentales datant des siècles précédents, et donc plus proches des réalités constitutionnelles et politiques qui ont existé dans l'Empire, à l'exception de la Bulle d'or, parce que l'élection de l'empereur se déroulait toujours selon les règles qu'elle avait prescrites.

Il faut noter que le baron Jean-François de Spon publia, dans les années 1740, en langue française, dans la ville de l'élection des rois des Romains et des empereurs du Saint Empire romain germanique, à Francfort-sur-le-Main, les capitulations impériales de Charles VII et de François Ier. L'édition de la première capitulation fut imprimée deux fois, en 1742 et $1743^{359}$, l'édition de la seconde capitulation parut en $1746^{360}$. S'il est permis de dire que Jean Heiss fit connaître la paix de Westphalie aux Français, on doit tenir Jean-François Spon pour celui qui propagea la connaissance des capitulations impériales des années 1740 en France. À propos de l'auteur ou, pour mieux dire, du traducteur des deux capitulations de Charles VII et de François Ier, on doit tout d'abord noter qu'il s'était aussi fait connaître comme traducteur de Pufendorf, dès 1728. À l'époque, il avait également publié une traduction française de la capitulation impériale de Charles VI. Ces traductions étaient accompagnées de notes de bas de page politiquement tendancieuses, qui valurent à leur auteur l'animosité des Impériaux et l'interdiction (sinon effective, du moins théorique) de son livre en France ${ }^{361}$. Ce personnage intéressant, qui servit d'intermédiaire culturel entre la France et le Saint-Empire, naquit à Strasbourg, le 10 octobre 1696. Il était le fils de Jean-Henri Spon (ou Spohn), un juriste originaire de Molsheim, qui, après des études de droit à Strasbourg, était devenu bailli du directoire de la noblesse immédiate de Basse-Alsace

359 [Jean-François, baron DE SPON], La Capitulation de l'Empereur Charles VII. Avec des remarques instructives touchant l'état et le gouvernement actuel de l'Empire, ainsi que sur les questions du droit public d'Allemagne les plus remarquables, Francfort/M. 1742; autre édition Francfort/M. 1743. Les errata de l'édition de 1742 n'ont pas été corrigés dans celle de 1743; l'éditeur s'est contenté de reproduire la liste des vingt-deux corrections, placée après la p. $\gg 506 \ll[=482]$, comme dans la première édition.

360 [ID.], La Capitulation de l'Empereur François combinée, avec la capitulation de l'Empereur Charles VII. qui en fait la base et ou on a joint des remarques historiques et politiques, Francfort/M. 1746. Il s'agit en fait d'une réimpression de l'ouvrage précédent, auquel on a ajouté des extraits de la capitulation de François Ier et d'autres pièces. Au lieu de corriger les errata des éditions de 1742 et de 1743 , on s'est contenté de reproduire la liste des vingt-deux corrections, placée après la p. " 506 « $[=482]$, comme dans les éditions antérieures.

${ }^{361}$ Samuel DE PUFENDORF, Etat de l'Empire d'Allemagne [...], 2 parties, Strasbourg 1728. Pour sa traduction de Pufendorf, à laquelle Spon a d'ailleurs ajouté une traduction française de la capitulation de Charles VI (seconde partie, p. [1]-180, version française de la capitulation de Charles VI avec les explications très longues du traducteur), cf. le chapitre VIII. 
et s'était établi à Strasbourg, à la fin du XVIIe siècle, après avoir été nommé directeur de la chancellerie municipale. Le grand-père de Jean-François Spon était Christophe Spon, receveur du grand chapitre de la cathédrale de Strasbourg. JeanFrançois était donc de souche alsacienne ${ }^{362}$ et issu d'une famille de juristes. En 1732, il épousa à Strasbourg Anne Marie Françoise Gelb, fille de François Nicolas Gelb, membre du grand sénat de la ville. Par ses origines et par son mariage, JeanFrançois appartenait donc aux patriciens de Strasbourg. Comme son père, il fit des études de droit dans sa ville natale et entama une brillante carrière de juriste et de diplomate. Son origine alsacienne et ses études de droit à Strasbourg firent de lui un exemple typique d'un médiateur culturel faisant connaître le droit public germanique aux Français. Dès le début de sa carrière, Spon passa en effet pour un excellent connaisseur du droit allemand ainsi que de l'histoire et des affaires politiques du Saint-Empire. À Strasbourg, Spon accéda au poste de préteur royal. Au moment de l'interrègne qui suivit la mort de Charles VI (1740-1742), Spon fut envoyé comme conseiller à l'électeur de Bavière, soutenu par la France. Ce candidat remporta l'élection contre les Habsbourg et monta sur le trône sous le nom de Charles VII (1742-1745). Pour la première fois depuis la fin du Moyen Âge, la couronne impériale échappa aux Habsbourg. Ce fut un succès éclatant pour la diplomatie française. Spon avait été chargé de sa mission en Bavière par le cardinal de Fleury, ministre d'État de Louis XV. En 1728, c'est au même cardinal de Fleury que Spon aurait dédié sa traduction de Pufendorf. Après l'élection de Charles VII, Spon devint le secrétaire intime du nouvel empereur et se vit conférer le titre de baron. Par la suite, Charles VII le chargea de plusieurs missions diplomatiques. Il l'envoya comme résident impérial à Paris en janvier 1743, puis à Berlin au mois d'août. Spon resta dans la capitale du Brandebourg même après la mort de Charles VII, en qualité de ministre de Bavière. À la fin de la guerre de Succession d'Autriche, en 1748, Spon participa en la même qualité aux conférences pour la paix d'Aix-la-Chapelle. Après son retour dans sa ville natale, l'ancien diplomate obtint la charge de directeur de la chancellerie municipale ainsi que celle de syndic royal, en 1759, et, l'année suivante, se fit immatriculer au corps de la noblesse immédiate de Basse-Alsace. En 1773, il décéda à Strasbourg. Son fils François-Nicolas, licencié en droit de la faculté de Strasbourg, fut le dernier à exercer la charge, avant la suppresion de celle-ci sous la Révolution, de premier président du conseil souverain d'Alsace, comme successeur de FrançoisHenri de Boug, que nous avons déjà rencontré comme éditeur de la paix d'Osnabrück $^{363}$. Jean-François Spon était donc un bon connaisseur des affaires de l'Empire et surtout un proche collaborateur de Charles VII, dont il traduisit la capitu-

$362 \mathrm{Par}$ son ancienneté, cette famille était établie dans l'électorat de Mayence, probablement dans la région de la Bergstraße, route entre Darmstadt et Heidelberg, mais elle avait migré en Alsace avant 1627.

363 Après quelques années d'exil en Angleterre, François-Nicolas de Spon renoua avec la tradition fondée par son père Jean-François: il passa au service de la Bavière en sa qualité de conseiller privé du prince-électeur. En 1800, il mourut à Londres. 
lation. En revanche, il s'était brouillé avec la cour de Vienne en 1728, au cours de l'affaire de sa traduction du fameux »Etat de l'Empire « de Pufendorf ${ }^{364}$.

Au début, le projet de l'édition des capitulations de Charles VII et de François Ier fut une entreprise personnelle de Spon, comme l'explique une »Lettre du Libraire à l'Auteur «, signée François Varrentrapp ${ }^{365}$. Selon cette lettre, Spon avait remis son manuscrit à cette maison d'édition pour en faire tirer une vingtaine d'exemplaires, à ses propres frais. Or, selon Varrentrapp, ce livre, finalement, bien que publié en Allemagne, cherche une grande partie de son public en France même; ce témoignage de l'éditeur peut servir de preuve au fait que les éditeurs tenaient la publication d'une version française d'une capitulation impériale pour une entreprise éditoriale rentable. Or, même dans les éditions publiées entre 1742 et 1746, Spon n'a pas seulement traduit les capitulations: il y a ajouté des notes qui ont particulièrement retenu l'intérêt de l'éditeur, car elles dépassent le simple commentaire de ces capitulations, examinant les caractères réguliers et irréguliers de la Constitution du Saint-Empire. En ce qui concerne le texte même des deux capitulations, un »Avis« au lecteur annonce qu'elles sont, pour la plupart des articles, identiques. Les observations précises de Spon témoignent du collationnement exact dont le manuscrit, confronté aux originaux allemands, à fait l'objet avant sa publication.

Par ailleurs, la situation que l'on n' avait plus connue depuis de nombreuses décennies, et qui se présenta avec la mort de Charles VI, en 1740, et l'interrègne qui la suivit, focalisa l'attention des Français sur un aspect qui ne passait pas, en règle générale, au premier plan de leurs préoccupations à l'égard de l'Allemagne: la vacance du trône impérial. Si la situation n'était pas inédite, on put surtout trouver des réponses aux questions qui se posèrent alors, dans les lois fondamentales de l'Empire, surtout dans la Bulle d'or et dans les capitulations impériales. C'est le continuateur de Dumont, Jean Rousset ${ }^{366}$, qui, d'après Barbier ${ }^{367}$, composa un

364 Pour la biographie de Spon et pour sa famille, cf. Jean-Marie ScHMITT, article »Spon, Jean François, baron de«, dans: NDBA, t. 35 (2000), p. 3708; ID., article "Spon (Spohn), Jean Henri火, dans: NDBA, t. 35 (2000), p. 3708; ID., article »Spon, François Nicolas, baron de «, dans: NDBA, t. 35 (2000), p. 3708; Seymour, baron DE SPON, Les barons de Spon d'Alsace, dans: Annuaire de la Société historique et littéraire de Colmar XXVI (1976-1977), p. 49-56 (avec la reproduction d'un portrait, p. 51). À l'exception de celle du baron de Spon, toutes ces contributions n'évoquent point la traduction de 1728 , pas plus que ses répercussions diplomatiques. SPON, Les barons, p. 52, mentionne brièvement la traduction et l'annotation de Pufendorf publiées en 1728. Pour une notice biographique de Spon, cf. également AE, MD Alsace 37 fol. 405-408; des lettres de Spon (fol. 398-404 et fol. 409-423) donnent des informations supplémentaires sur sa vie.

365 Nous nous référons ci-après, sauf indication contraire, à l'édition de 1746, car elle est la plus complète. La lettre s'y trouve après le frontispice, p. [I]. La même lettre se trouvait également dans l'édition de 1742 , p. [I].

366 En 1739, il publia un supplément au »Corps universei« de Jean Dumont.

367 L'auteur d'après BARBIER, Dictionnaire des ouvrages anonymes, t. III, col. 230 . Cf. la note manuscrite dans l'exemplaire M.14820, avant le frontispice. Barbier connaît une autre édition de 1742 que nous n'avons pas vue. Comme la première édition, elle serait parue chez P. Mortier à Amsterdam. En revanche, il ignore celle de 1745 (voir ci-dessous). 
ouvrage assez volumineux sur ce problème ainsi que sur les autres questions liées à l'élection de l'empereur et aux électeurs du Saint-Empire. Ce traité fut publié à deux reprises, sous le titre de "Memoires instructifs «, à Amsterdam, en 1741 et en 1745368. Il est composé de quatre mémoires différents. Le troisième mémoire intitulé »De la Capitulation Impériale \& des Interêts de l'Empire ${ }^{369}$ donne la version française de la capitulation de l'empereur Charles Quint ${ }^{370}$, pièce que Rousset a tirée de Ziegler ${ }^{371}$, comme il explique lui-même ${ }^{372}$, ainsi que la traduction en français de la capitulation de Charles VI ${ }^{373}$. Au total, Rousset reproduit trois lois fondamentales: la Bulle d'or et les capitulations impériales de Charles Quint et de Charles VI, ainsi que le projet de la capitulation perpétuelle de 1711. Rousset considère le Saint-Empire comme une aristocratie, conservant seulement en apparence quelques caractéristiques de la monarchie ${ }^{374}$, alors que du temps de l'instauration de l'empire sous Jules César et de sa restauration sous Charlemagne, il était monarchique ${ }^{375}$. La localisation de la souveraineté correspond à la théorie de Bodin: en Allemagne, elle résiderait dans la diète ${ }^{376}$, terme qui, pour Rousset, est synonyme d'Empire ${ }^{377}$. L'interprétation de la capitulation impériale constitue un élément essentiel dans l'argumentation de Rousset, qui, en vertu de l'article XX

368 [Jean RouSSET], Memoires instructifs, I. Sur la vacance du trone imperial. II. Les droits des electeurs et de l'Empire [...], 1 vol., Amsterdam 1741; 2 t. en 1 vol., ibid. 1745. La seule différence majeure entre l'édition de 1741 et celle de 1745 est l'adjonction de deux tables des matières: au t. I, entre l'avertissement et la p. [1], et au t. II, vers la fin, entre les pages 124 et 125.

369 RousseT, Memoires (1741), p. 132-263; ID., Memoires (1745), t. I, p. 156-313 (titre en principe identique, ponctuation et accentuation différentes).

370 RousSET, Memoires (1741), p. 138-152; ID., Memoires (1745), t. I, p. 164-181.

${ }^{371}$ Ziegler a publié cette pièce en 1711, cf. Christoph ZIEGLER, Wahl=Capitulationes, Welche mit denen Rőmischen Kăysern und Kŏnigen/ Dann des H. Ro̊m. Reichs Churfürsten [...] auffgerichtet/ vereiniget und verglichen [...], Francfort/M. 1711, p. 7-22. Aujourd'hui, on se reportera à l'édition de WALDER, Kaiser, Reich und Reformation 1517 bis 1525, p. 2534.

372 Rousset, Memoires (1741), p. 138; ID., Memoires (1745), t. I, p. 164.

373 RousseT, Memoires (1741), p. 155-238; ID., Memoires (1745), t. I, p. 185-284. Pour une traduction de la Bulle d'or et de la capitulation perpétuelle, cf. ID., Memoires (1741), p. 9-80, 293-362; ID., Memoires (1745), t. I, p. 12-93, t. II, p. 35-113.

$374 »$ Dans le Gouvernement de l'Empire ce qu'il y a de Monarchique, semble n'y avoir été conservé que pour donner plus de lustre $\&$ plus de force $\&$ de grandeur à l'Aristocratie«, RousSET, Memoires (1741), p. 119. L'auteur admet, toutefois, que cette question est litigieuse parmi les juristes de l'Empire: »Les Savans, dans les Universitez d'Allemagne, ont longtems disputé sur la nature du Gouvernement de l'Empire. Quelques-uns ont soutenu qu'il étoit Monarchique, d'autres ont démontré qu'il étoit Aristocratique«, ibid., p. 116-117. Â cet endroit, il omet la théorie du gouvernement mixte, mais il mentionne ailleurs son existence (par exemple, ibid., p. 119).

375 Cf. ibid., p. 117-118.

376 »Nous avons dit que la Puissance Souveraine, ou la Souveraineté de l'Allemagne réside dans l'assemblée de ses Etats convoqués en Diète«, ibid., p. 120.

377 „Je crois qu'en voilà assez pour prouver que la Souveraineté réside dans l'Empire; ou, si l'on veut, dans la Diète générale de l'Empire, dont les Constitutions \& les Recès sont les Loix fondamentales, avec certains Traitez solemnels, comme celui de Westphalie«, etc.; ibid., p. 127-128 (citation p. 127). 
de la capitulation de Charles VI, attribue le droit de proscription (souvent mentionné pour marquer la soumission des ordres au pouvoir de l'empereur) aux états eux-mêmes ${ }^{378}$.

Nous pouvons constater que, surtout au XVIII' siècle et tout particulièrement depuis 1740 , les capitulations impériales et l'élection du roi des Romains constituèrent des objets de prédilection dans les ouvrages français concernant le droit public germanique. Parfois, ces publications se penchent sur un problème très précis. C'est le cas, par exemple, de la »Représentation impartiale«, une brochure publiée à titre anonyme à La Haye, en $1751^{379}$. Cette publication s'inspire très certainement des bruits qui couraient alors en Allemagne au sujet d'une éventuelle élection d'un roi des Romains ${ }^{380}$. L'auteur de la brochure se pose une question très concrète: la question de savoir si, outre le collège électoral qui avait le droit exclusif à l'élection d'un empereur, celui des princes pouvait concourir à l'examen de la question préalable de savoir s'il convenait de passer à l'élection d'un roi des Romains, question que les juristes de l'Empire appelait en latin la question »an«381. Il s'agissait d'un problème fondamental qui était souvent âprement discuté par les électeurs et par les princes. En fait, ces derniers cherchaient à gagner de l'influence sur l'élection impériale en s'associant aux discussions préalables, alors que les électeurs voulaient éviter que les princes n'empiètent sur leurs prérogatives qu'ils tenaient pour exclusives. Selon cet auteur, les journaux allemands et français avaient publié des articles sur ce problème, dont il ne partageait pas les conclusions. Il cherche à défendre sa propre solution du problème en fondant son argumentation sur l'examen juridique des lois de l'Empire et sur l'histoire. Cet auteur conclut que tout ce qui touche à l'élection impériale est une affaire entièrement et exclusivement réservée aux seuls électeurs, sans que les princes ne puissent légitimement invoquer le droit de concourir ne serait-ce qu'à la question de savoir s'il convient véritablement d'élire un nouvel empereur ${ }^{382}$. Enfin, il estime que la situation dans laquelle le Saint-Empire se trouvait en 1751 demandait que le collège électoral élût un roi des Romains.

C'est cet intérêt particulier que les auteurs de langue française et leur public apportèrent à l'élection impériale qui explique aussi le nombre tout à fait considérable des traductions françaises des capitulations des empereurs. Car, outre les éditions déjà évoquées, beaucoup d'auteurs et d'éditeurs firent publier leurs traductions. Parmi les traductions françaises intégrales des capitulations impériales, il

378 Ibid., p. 126-127. Au sujet de sa perception du gouvernement de l'Allemagne, voir aussi, dans l'édition de 1741 , p. 116-130, 157, 160-162,169-170 et 248 .

379 Représentation impartiale de ce qui est juste à l'égard de l'élection d'un Roi des Romains selon les loix et les coutumes du Saint Empire Romain, La Haye 1751.

$380 \mathrm{Cf}$. le début de cet écrit (divisé en vingt et un paragraphes ou chapitres), qui évoque ces rumeurs. Le fait que l'auteur explique qu'il ne publie pas son petit livre pour ces discussions actuelles apparaît comme une convention littéraire, cf. ibid., p. [3].

381 „Si outre le Collège des Electeurs, celui des Princes n'étoit pas autorisé de concourir à une affaire de cette conséquence, du moins quant à la question AN?«, ibid.

382 Cf. ibid., p. 63. 
faut encore mentionner la version publiée par Scheid, qui, en 1754, publia la capitulation de François Ier de $1745^{383}$. Il y ajouta les passages des deux capitulations précédentes (c'est-à-dire de celles de Charles VI de 1711 et de Charles VII de 1742) qui avaient été omis ou modifiés ${ }^{384}$. En revanche, Scheid a mis en italique les passages ajoutés ou modifiés dans la capitulation de François $\mathbf{I}^{\mathrm{er}}$ par rapport à celles de ses deux prédécesseurs ${ }^{385}$. Comme pour la paix de Westphalie et la Bulle d'or, il donne des »traductions corrigées « ${ }^{386}$. L'auteur note que la capitulation de François Ier »est presque conforme« à celle de Charles VII. En revanche, il constate que plusieurs additions et modifications distinguent ces deux capitulations de celle de Charles VI ${ }^{387}$. Cette édition n'est pas annotée. En principe, sa traduction correspond à celle qui a été publiée par Spon. Mais, comme pour le traité d'Osnabrück, Scheid a évidemment revu la traduction de Spon et, très certainement, il l'a confrontée au texte original latin, puisque certaines corrections témoignent de sa réflexion sur la traduction de concepts juridiques ${ }^{388}$. Il corrige aussi certaines omissions de Spon ${ }^{389}$. En conclusion, il faut admettre que sa traduction est plus précise et plus réussie que la version publiée par $\operatorname{Spon}^{390}$. Avant Scheid, Mauvillon avait repris la traduction de la capitulation de François Ier de l'ouvrage de Spon, l'insérant dans son »Droit public germanique« paru en $1749^{391}$. Dans la plupart des cas, Mauvillon avait conservé les passages moins exacts de la version de Spon que Scheid corrigea quelques années plus $\operatorname{tard}^{392}$.

383 SCHEID, Traité sistématique, t. IV, p. [1]-142.

384 Ibid., p. 143-146 et p. 147-148.

385 Cf. ses explications à ce sujet dans l'»Avertissement« au lecteur, ibid., p. [III]-VI, ici p. [III]-IV.

386 Ibid., p. [III]. Pour sa traduction de la Bulle d'or, cf. ibid., p. 327-483.

387 Ibid., p. [III].

388 C'est, par exemple, dans l'article Ier, alinéa II que Scheid opte pour l'emploi du mot »Droits« au lieu de »jurisdiction« chez Spon. Cf. SPON, Capitulation, p. 33; ScHEID, Traité sistématique, t. IV, p 4 .

389 On note que Spon oublia, par exemple, de mentionner le droit civil dans le dernier article de la capitulation, alinéa VII. Cette référence fut rajoutée par Scheid. Dans le même article, même alinéa, Scheid traduit de manière plus exacte la formule par laquelle l'empereur s'obligea de respecter et d'exécuter les articles de sa capitulation. Cf. SPON, Capitulation, p. 436; SCHEID, Traité sistématique, t. IV, p 138.

390 Pour démontrer la plus grande exactitude de la version de Scheid, on peut également alléguer le début de l'article XVI relatif à la justice. Selon la traduction de cet auteur, François Ier promit de procurer que dans le Saint-Empire, la justice "prenne, ait \& conserve son cours légitime«. Spon raccourcit ce passage en faisant promettre à l'empereur de veiller à ce que la justice y »ait son cours légitime«. À l'alinéa II, Scheid remplace l'expression »l'Empire d'Allemagne« par »l'Empire de la Nation Allemande«, une traduction qui est plus proche de la formule de chancellerie utilisée en Allemagne. Cf. SPON, Capitulation, p. 309-310; SCHEID, Traité sistématique, t. IV, p 82-83.

391 [MAuvillon], Le Droit public germanique (1749), t. Il, p. [1]-138. Cette édition ne comporte pas de commentaire. Cf. aussi l'édition de 1756, t. II, p. 1-138.

392 En ce qui concene les exemples que nous avons allégués ci-dessus, la traduction de Mauvillon correspond dans presque tous les cas à celle de Spon. En revanche, on note une exception où elle n'est ni tout à fait conforme à la version de Spon ni à celle de Scheid: Mau- 
Outre les différentes capitulations que nous venons de nommer, on traduisit également en français l'édition de toutes les capitulations et du projet pour une capitulation perpétuelle que Johann Christian Müldener avait publiée en allemand, en $1697^{393}$. La version française parut en $1750^{394}$. Cet ouvrage est composé de trois parties: la première consiste dans la comparaison des dix premières capitulations impériales qui datent de la période 1519-1690 (traduction synoptique). La deuxième partie est également comparative: elle confronte la capitulation de Charles VI au projet pour une capitulation perpétuelle de $1711^{395}$. Enfin, la troisième partie contient les capitulations de Charles VII et de François Ier. Les deuxième et troisième parties sont donc formées par des continuations de l'ouvrage de Müldener. Selon l'approbation datée à Paris, le 4 mars 1750 , et signée Secousse, ainsi que selon le privilège du roi, datant du 19 du même mois ${ }^{396}$, l'ouvrage fut traduit de l'allemand par un certain Besset de La Chapelle ${ }^{397}$. On sait peu de choses sur ce personnage, si ce n'est que, outre l'ouvrage de Müldener, cet écrivain du XVIII ${ }^{e}$ siècle traduisit à titre anonyme plusieurs livres anglais en français, par exemple la »Correspondance du chevalier de Rob-Cecil avec Jacques VI«, une »Histoire d'Écosse sous le règne de Marie Stuart et de Jacques VI« ainsi qu'un »Tableau historique et politique de la Suisse « ${ }^{398}$. À sa traduction du livre de Müldener, le traducteur joignit un »Discours Preliminaire«, dans lequel il donnait un aperçu du droit allemand, en particulier de la place des capitulations impériales dans le système du droit public du Saint-Empire ${ }^{399}$.

Il est évident que l'idéal de traduction connu sous le nom des "belles infidèles" ne fut pas retenu comme idéal de la traduction de ce genre d'acta publica. Dans la première phrase de son "Discours Preliminaire«, le traducteur lui-même donne une définition à la fois de sa méthode de traduction et des capitulations impériales:

villon écrit »Jurisdictions« au pluriel, au lieu de »jurisdiction« au singulier chez Spon et "Droits« chez Scheid, ibid., p. 4.

393 Johann Christian MüLDENER, Capitulatio harmonica: d.i. Der Allerdurchlauchtigsten Rómischen Kåyser und Ko̊nige [. . . W Wahl=Capitulationes [...], 2 t. en 1 vol., Hall in Sachsen 1697

394 [Johann Christian] MüLDENER, Capitulation harmonique [...], continuée jusqu'au tems présent: Ou traduction exactement litterale, et mot pour mot; et concordance generale de toutes les capitulations des Empereurs [...], Paris 1750.

395 Le traducteur a remplacé par cet acte plus moderne le projet pour une capitulation perpétuelle publié par Müldener en 1697, qui n'était plus d'actualité au temps de la traduction. Pour une traduction de la capitulation de Charles Quint, cf. ibid., première partie, p. LI[I]JLXIIJ.

396 Ces deux pièces se trouvent dans MüLDENER, Capitulation harmonique, placées au début du volume, entre la »Table des Articles« et le »Discours Preliminaire«.

397 Même au dépôt des archives on recourait à l'édition des capitulations impériales par Besset de La Chapelle; cf. la note "Sur l'introduction des nouveaux princes dans le collège des princes de l'Empire «, sous-titre: »Note sur l'article Ier $\$ 5$ de la capitulation de François $I^{\mid{ }^{e r}} \ll$; cette note date probablement de 1751 (AE, MD All. 107 fol. 305).

$398 \mathrm{La}$ »Nouvelle Biographie générale« donne les initiales de son prénom: N.-P. Cf. l'article biographique »Besset de la Chapelle (N.-P.)«, dans la Nouvelle Biographie générale, t. 5 (1853), col. 812-813.

399 MüldDENER, Capitulation harmonique, p. J-XLIIJ. 
"J'entreprens de donner une traduction exactement littérale \& mot pour mot, du pacte que les Electeurs, tant en leur nom qu'en celui de tous les Etats de l'Empire, font avec un Roi des Romains lors de son Election «400. Le début de ce discours dévoile l'esprit méthodique du traducteur. Sur le plan de la traductologie, on note qu'il adhère à l'idéal de la traduction »mot pour mot«, qui, à cette époque, était moins en vogue en France qu'en Allemagne, du moins dans la littérature, mais qui semblait devoir s'imposer dans les deux pays pour traduire les documents à caractère officiel. Besset, pour sa part, défend cette approche comme l'unique manière de bien traduire les actes publics en général, et en particulier les capitulations impériales: »L'élégance, dans la traduction d'un Acte, c'est la fidélité « «01. Ce traducteur définit le Saint-Empire comme un »gouvernement mixte « 402 .

Dans son »Histoire générale d'Allemagne«, un autre exemple est donné par Joseph Barre, qui reproduit, en 1748, une traduction française de la capitulation impériale de Charles Quint, en tant que première capitulation jurée par un nouveau roi des Romains lors de son élection et »fondement de celles qui ont été ensuite jurées par les Empereurs « ${ }^{403}$. On constate que la traduction publiée par Barre ne correspond pas à la version française publiée par Rousset, en 1741 et en 1745 , ni à la traduction publiée par Besset de La Chapelle, en 1750. Les trois traductions sont indépendantes les unes des autres. La version de Barre contient une erreur importante dans l'article concernant les langues admises dans l'administration publique de l'Empire ${ }^{404}$. Dans la traduction des lettres patentes de Charles Quint,

400 Ibid., p. J. Au sujet de son idéal et de sa méthode de traduction, cf. également ibid., p. XXVIIJ-XXIX et XLV-XLVIJ.

401 Ibid., p. XXVIIJ.

402 Ibid., p. XIJ.

403 Cf. [Joseph] BaRRE (S.J.), Histoire générale d'Allemagne [...], 10 t. en 11 vol., Paris 1748 , ici t. VII, deuxième partie (placée au début du vol. VIII), p. 1107-1113, citation p. 1107, n. a; édition partielle comprenant le préambule et les vingt-cinq premiers articles de cette capitulation que, au total, Barre a divisée en trente-quatre articles. Il ne renvoie à aucune édition française antérieure, mais à deux éditions de Goldast et à une reproduction de Limnaeus. Pour l'importance de cette capitulation, il se réfère à Pfeffinger (à son sujet, voir le chapitre VIII).

${ }^{404}$ Barre traduit ainsi cet article: „Dans les écritures \& les actes de l'Empire, nous ne nous servirons d'autre langue que de l'Allemande ou de la Latine, si ce n'est pour les endroits qui sont hors de l'Empire où d'autres langues sont en usage«, BARRE, Histoire générale d'Allemagne, t. VII/2, p. 1111. Or, dans cet article de la capitulation de Charles Quint, le lieu n'est en aucune manière précisé, mais il est certainement question des endroits dans l'Empire où l'on parle une autre langue que l'allemand et le latin. C'est donc la traduction de Rousset qui est correcte: $»$ Nous ne devons \& ne voulons pas nous servir d'aucune autre langue, que de l'Allemande, ou de la Latine dans tous les Actes \& Ecrits publics. Si ce n'est, que cela arrive dans des Endroits, où ordinairement une autre langue seroit en usage, alors il est permis à Nous \& aux nôtres, de Nous y servir aussi de la langue de ce paiss" (ROusSET, Memoires instructifs, 1741, t. I, p. 144-145). Cette traduction correspond très exactement à l'original allemand: »Darzue in schriften und handlungen des reichs kain ander zunge oder sprach gebrauchen lassen, wann die Teutsch oder Lateinisch zung; es wer dann an orten, da gemeinlich ein andere sprach in ubung und gebrauch stuend, alsdann mugen wir und die unsern uns derselbigen daselbs auch behelfen «, WALDER, Kaiser, Reich und Reformation, p. 25-34 (capitulation de Charles Quint), ici p. 29. 
Barre met en caractères italiques le passage essentiel définissant la nature de la capitulation de l'empereur, qui fut conclue "par forme de contract \& d'obligation réciproque ${ }^{405}$. Ainsi, Barre donne une définition du caractère juridique de la capitulation comme contrat.

Parmi les capitulations impériales postérieures à 1648 et antérieures à 1756, on constate qu'un seul acte ne fut pas publié indépendamment en version française. Il s'agit de la capitulation de Ferdinand IV, mort en 1654, avant d'accéder à l'empire. Cependant, cette capitulation faisait partie de la "Capitulation harmonique « de Müldner, traduite en français par Besset en 1750, et Jean Dumont offrait une version latine de la capitulation de 1653 au public européen. Même cette capitulation d'un roi des Romains qui n'accéda jamais à l'empire était donc accessible aux élites françaises dans une version française comme partie de l'édition synoptique des capitulations de tous les empereurs des XVIe et XVII siècles, et dans une traduction particulière latine pour ceux qui lisaient le latin ${ }^{406}$.

\section{Les traductions françaises de la Paix publique de 1495, du traité de Passau de 1552 et de la paix de religion d'Augsbourg de 1555}

La Paix publique »perpétuelle« de 1495 (Ewiger Reichslandfriede) constitue une exception parmi les lois fondamentales: selon nos recherches, aucune traduction française n'en fut publiée. En revanche, certains auteurs en ont résumé les principaux articles ${ }^{407}$. Toujours est-il que dans les livres imprimés on ne trouve qu'une traduction française de la confirmation de la Paix publique insérée dans le recès de l'Empire qui fut promulgué à Augsbourg, en 1548408. L'auteur de cette traduction, Simon Jacquet, traduit le terme allemand "Allgemeiner Landfrieden" par "Paix generale de l'Empire". Cependant, la paix de 1495 fut bien traduite, car on peut en trouver des copies manuscrites. La copie la plus complète date de la seconde moitié du XVIe siècle. Elle est conservée dans la collection Dupuy de la

405 BARRE, Histoire générale d'Allemagne, t. VII/2, p. 1107.

406 Cet acte du 26 juin 1653 est publié en latin dans Dumont, Corps universel, t. VI/2 (1728), document $n^{\circ}$ XVII, p. 59-65. Comme éditions antérieures, Dumont signale celle de Thuldenus, dans sa »Tractatuum Historico-Politicorum Parte II.«, dont il tira le texte latin, et, en allemand, les »Wahl=Capitulationes « de Ziegler, le »Jus publicum romano germanicum « de Limnaeus et les »Acta Publica« de Londorp. Cf. également ZIEGLER, Wahl=Capitulationes, p. 157-199.

407 Cf., par exemple, SPON, Capitulation, p. 68-69, n. o; ibid., p. 69-70, n. p, le commentateur de la capitulation de Charles VII résume les principales clauses de la paix de religion de 1555 (par erreur, il mentionne comme lieu de conclusion la ville de Passau, où fut signé le traité de 1552, au lieu de celle d'Augsbourg).

408 JACQUET, Droit public d'Allemagne, t. IV, p. 333-374; ID., Recueil precieux, t. I, p. 333374. Ces ouvrages offrent aussi des traductions de la paix publique de 1548, de l'ordonnance d'exécution de 1555 et du dernier recès de 1654 ainsi que de la capitulation de Joseph II de 1764, cf. ID., Droit public d'Allemagne, t. IV, p. 333-374, t. V, p. 1-38, t. VI, p. 206-347 et 348-488; ID., Recueil precieux, t. I, p. 333-374, 1-38 (deuxième pagination), t. II, p. 206347 et $348-488$. 
Bibliothèque nationale, et prend aussi en considération les lois impériales postérieures à 1495 qui devaient affermir la Paix publique ${ }^{409}$. Or, dans la période qui va de 1648 à 1756 , les auteurs ont certes parlé de cette paix publique, mais leur attention ne s'est jamais vraiment attardée sur ce document.

L'on doit noter que, outre la traduction partielle de la Paix publique de l'Empire de 1495 , ce manuscrit contient une traduction de certains articles de la Bulle d'or et une version française des articles d'Augsbourg de 1555. Les traductions de ce dernier document sont très rares. Le droit religieux allemand compte, en effet, parmi les sujets que les auteurs de langue française ont tendance à négliger. Le traité de Passau a d'abord été publié par Jean Heiss, en 1684, dans l'»Histoire de l'Empire «. Sa traduction ${ }^{410} \mathrm{a}$ subi de très légères modifications dans les rééditions datant du XVIIIe siècle ${ }^{411}$. Par exemple, on note que le titre est très légèrement différent à celui des éditions du XVII e siècle ${ }^{412}$. À la traduction proprement dite, ses continuateurs (en l'occurrence Vogel ou les éditeurs néerlandais) ont ajouté des "Remarques « sur la paix de religion et sur le traité de Passau, en $1733^{413}$. En 1731, Vogel y ajouta également un commentaire tendancieux sous forme de notes de bas de page qui expliquent, par exemple, que depuis la bataille de Mühlberg, l'intention de Charles Quint avait été de mettre sous son joug tous les princes d'Allemagne et de casser leurs privilèges, de sorte que »l'Allemagne est redevable au Roi de France de ce qui lui reste de liberté « 414 . Le commentateur prétend également que »les Princes d'Allemagne avoient cedé au Roi« Henri II les villes de Metz, Toul, Verdun et Strasbourg ${ }^{415}$. La traduction de Heiss ${ }^{416}$ a été reprise

409 BNF, Coll. Dupuy 587 fol. 22-58'. Pour des raisons paléographiques et orthographiques, on peut dater cette copie de la seconde moitié du XVI ${ }^{e}$ siècle ou du tout début du XVIIe. C'est donc la plus ancienne traduction de la Paix publique de 1495 que nous ayons retrouvée au cours du dépouillement des fonds de bibliothèques et d'archives, bien antérieure à la première traduction imprimée par nous repérée, dans l'»Histoire de l'Empire « de Jean Heiss, dont la première édition fut publiée en 1684.

410 Nous la citons d'après l'édition publiée en 1685: HeIss, Histoire de l'Empire (1685), t. III, p. 272-293.

411 Par la suite, nous citerons la dernière réédition, qui date de 1733 , édition in- $8^{\circ}$. Pour le traité de Passau, cf. cette dernière édition, t. VI, p. 481-514.

412 Cf. HeIss, Histoire de l'Empire (1685), t. III, p. 272: »Transaction Conclûë \& ratifiée à Passau le deuxiéme jour d'Aoust l'an mil cinq cens cinquante deux, sous l'autorité de Charles V. Empereur toûjours Auguste, entre Ferdinand Serenissime Roi des Romains, \&c. \& quelques Etats d'Allemagne, dite la paix publique «. Édition de 1733 (in-8 ${ }^{\circ}$ ), t. VI: »Transaction Concluë \& ratifiée à Passau, sous l'autorité de Charles V. Empereur toujours Auguste, entre Ferdinand Sérénissime Roi des Romains, \&c. \& quelques Etats d'Allemagne, dite la Paix Publique. Le 2. d'Août 1552«. En outre, dans l'ouvrage original de Heiss, la fin du traité porte le titre »Signature«, mal à propos, puisqu'il ne s'agit pas des signatures, cf. HEIss, Histoire de l'Empire (1685), t. III, p. 292. Dans les éditions postérieures à 1700, ses continuateurs ont remplacé ce titre par la note suivante: »La conclusion du Traité est ainsi énoncée«; cf., par exemple, l'édition in $-8^{\circ}$ de 1733 , t. VI, p. 513.

413 Ibid., p. 515-519 (p. 400-401, dans l'édition in-40). Comme cette remarque sur la paix de religion et sur le traité de Passau ne se trouve pas dans les éditions antérieures à 1733, nous devons très certainement l'attribuer à Vogel ou aux éditeurs hollandais.

414 Cf. HeIss, Histoire de l'Empire (1731), édition in-40, t. III, deuxième partie, p. 30, n. b.

415 Cf. ibid., n. a. 
dans les ouvrages publiés par Jacquet à la fin de l'Ancien Régime: son »Droit public « 417 et son »Recueil precieux « ${ }^{418}$. La traduction est identique à la version publiée dans les rééditions de l'»Histoire de l'Empire " datant du XVIIIe siècle, à quelques exceptions près ${ }^{419}$. Jacquet a aussi repris les titres que Heiss avait donnés à chaque chapitre du traitét ${ }^{420}$, mais il a omis les notes de bas de page que ses continuateurs y avaient ajoutées. La version française publiée par Heiss est également mentionnée dans le »Corps universel «21. Des tomaison et pagination indiquées par Dumont il résulte que celui-ci consulta la deuxième ou la troisième édition de l'»Histoire de l'Empire«, publiées respectivement en 1685 et en $1694^{422}$.

La paix de religion d'Augsbourg, charte religieuse de l'Allemagne depuis $1555^{423}$, confirmée et élargie aux calvinistes par la paix de Westphalie, a aussi été

416 Cf. Heiss, Histoire de l'Empire (1685), t. III, p. 272-293.

417 JACQUET, Droit public d'Allemagne, t. IV, p. 375-403.

418 JACQUET, Recueil precieux, t. I, p. 375-403.

419 On note, par exemple, que le titre du document est plus proche de l'édition de 1733 de l'»Histoire de l'Empire « de Jean Heiss, mais qu'il en diffère très légèrement; cf. HEIss, Histoire de $1^{\prime}$ Empire (1733; in-8 ${ }^{\circ}$ ), t. VI, p. 481 (= t. II, p. 388, in-40); cf. également JACQUET, Recueil precieux, p. 375. Le titre du dernier paragraphe correspond également aux rééditions de l'»Histoire de l'Empire « datant du XVIII' siècle (par exemple, celle qui est citée ci-dessus, édition in- $8^{\circ}$ de 1733 ), qui diffèrent, à cet égard, de celles du XVII ${ }^{e}$ siècle, cf. JACQUET, Recueil precieux, p. 403.

420 À cet égard, on constate une seule différence mineure concernant le titre du chapitre $\mathrm{V}$, cf. HeIss, Histoire de l'Empire (1685), t. III, p. 285 (titre identique dans l'édition de 1733, in- $8^{\circ}$, p. 503); erreur dans JACQUET, Recueil precieux, p. 392. En outre, dans le titre du chapitre VIII, l'orthographe de la ville de Goslar est sensiblement différente, HeIss, Histoire de l'Empire (1685), t. III, p. 288: »Gollard«; l'édition in- $8^{\circ}$ publiée en 1733, p. 509 a la meilleure leçon: »Goslar «; JACQUET, Recueil precieux, p. 397: „Coslar «.

421 DumonT, Corps universel, t. IV/3 (1726), document $n^{\circ}$ XVIII, p. 42-47, ici p. 42. Dumont signale aussi plusieurs éditions latines et allemandes. Cet auteur imprime une version latine du traité. En revanche, on constate que pour la paix d'Augsbourg et la Declaratio Ferdinandea Dumont ne signale aucune édition française. Il publie ces documents respective-

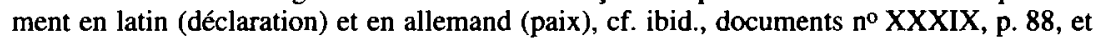
$\mathrm{n}^{\circ} \mathrm{XL}$, p. 88-93.

422 En effet, dans les deux éditions de HeIss, Histoire de l'Empire (1685 et 1694), le traité de Passau commence au t. III, p. 272.

${ }^{423}$ Pour les principaux ouvrages allemands et français sur la paix de religion d'Augsbourg, cf. les éléments de bibliographie cités par Thomas BrocKMANN, article »Religionsfriede«, dans: Lexikon für Theologie und Kirche, t. VIII, Fribourg/Brisgau ${ }^{3} 1999$, col. 1054. Pour les publications récentes, en particulier pour une évaluation des travaux de Konrad Repgen, cf. Martin HECKEL, Konfessionalisierung in Koexistenznöten. Zum Augsburger Religionsfrieden, Dreißigjährigen Krieg und Westfälischen Frieden in neuerer Sicht, dans: Historische Zeitschrift 280 (2005), p. 647-690. Pour l'histoire de la paix d'Augsbourg, on peut toujours se reporter au grand classique de Moriz RITTER, Deutsche Geschichte im Zeitalter der Gegenreformation und des Dreissigjährigen Krieges (1555-1648), 3 vol., Stuttgart, Berlin 1889-1908 (Bibliothek deutscher Geschichte, VIII; réimprimé à Darmstadt, en 1974), en particulier vol. 1. La meilleure analyse récente en français des pratiques de la paix religieuse

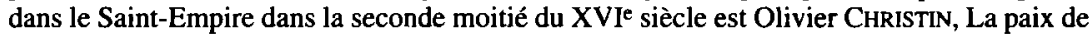
religion. L'autonomisation de la raison politique au XVI ${ }^{\mathrm{e}}$ siècle, [Paris] 1997. La commémoration du $450^{e}$ anniversaire de la paix d'Augsbourg en 2005 a donné lieu à un certain nombre de publications en Allemagne; cf., par exemple, Axel GoTTHARD, Der Augsburger Religi- 
traduite par Heiss et publiée en 1684; puis cette traduction a été reprise dans les différentes rééditions de son ouvrage ${ }^{424}$. Apparemment, la désignation de »Paix de religion " passait pour un concept spécifiquement allemand en France ${ }^{425}$. Avant la paix de Westphalie, on trouve aussi d'autres traductions françaises du titre de cette charte. En effet, dans la version française de l'édit de Restitution de 1629 publiée dans le »Mercure françois«, puis dans le »Corps universel« de Dumont, en 1728, plusieurs termes désignent cette paix d'Augsbourg: »Paix pour la religion «, »Traicté de Paix pour la Religion«, »Edict de Paix pour la Religion«, »Edict de Pacification pour la Religion ${ }^{426}$. Mais ces variantes étaient facilement compréhensibles. C'est la traduction de la paix de religion de Heiss (1684) qui s'imposa en France; en 1775, Boug la reproduisit au début de l'édition des »Ordonnances d'Alsace « qu'il avait entreprise ${ }^{427}$. Avec sa traduction de la paix de religion, Heiss trouva enfin un dernier successeur dans le personnage de Jacquet, qui a repris cette traduction pour l'insérer dans son "Droit public d'Allemagne« en $1782^{428}$, et dans son »Recueil precieux « des lois constitutionnelles du Saint-Empire en $1787^{429}$. Même le titre du document est identique ${ }^{430}$. Or, si dans l'ouvrage de Heiss les différents paragraphes ne sont pas numérotés, Jacquet a divisé le texte en vingt et un articles. En revanche, Heiss a indiqué les principaux objets des paragraphes en marge du texte et Jacquet a renoncé à ces notes marginales. Les deux auteurs reproduisent les paragraphes du recès d'Augsbourg concernant la paix religieuse ainsi que le préambule et la fin du recès, sans commentaire ni annotation.

onsfrieden, Münster 2004 (Reformationsgeschichtliche Studien und Texte, 148); ID., Der Augsburger Religionsfrieden und Franken, dans: Zeitschrift für bayerische Landesgeschichte 68 (2005), p. 555-572; Carl A. HofFMANN et al. (dir.), Als Frieden möglich war. 450 Jahre Augsburger Religionsfrieden. Begleitband zur Ausstellung im Maximilianmuseum Augsburg [16.6.-16. 10.2005], Ratisbonne 2005.

424 Cf. notamment HeISs, Histoire de l'Empire (1685), t. III, p. 294-305; (1733, in-80), t. VI, p. 520-535.

425 Au milieu du XVIIIe siècle, un historien de la paix des Pyrénées expliqua que »la convention de 1555 [...] est connue dans l'Empire sous le nom de paix de Religion « - cette remarque suggère qu'il n'en allait pas de même en France, cf. [Luc CoURCHETET D'EsNANS], Histoire des négociations, et du traité de paix des Pyrénées, Amsterdam, Paris 1750, t. I, p. 108.

426 Nous citons cette traduction d'après DumONT, Corps universel, t. V/2 (1728), document $\mathrm{n}^{\circ}$ CCCIX, p. 564-569, ici en particulier p. 567-569.

427 Boug D'Orschwiller, Ordonnances d'Alsace, t. I, p. XXXVIII-XLII (»Extrait Du Recès ou Résultat conclu entre Ferdinand, Roi des Romains, \& les États de l'Empire, en la Diete d'Ausbourg, l'an 1555, que l'on appelle communément La Paix De Religion «). Le texte est très exactement le même que dans l'ouvrage de Heiss; mais le premier président du conseil souverain d'Alsace a retiré les notes de bas de page, et dans le résumé des articles concernant les affaires séculières de l'Empire, Boug (p. XLII) écrit »particulieres « au lieu de "séculiéres « (cf. HeISs, Histoire de l'Empire [1733, édition in-8º], t. VI, p. 534 [= t. II, p. 407 dans l'édition in-4ㅇ); de plus, la fin de ce résumé diffère un peu du texte de Heiss, mais ces divergences n'ont aucun intérêt.

428 Cf. JACQUET, Droit public d'Allemagne, t. IV, p. 404-419.

429 Cf. ID., Recueil precieux, t. I, p. 404-419.

430 Abstraction faite des différences d'orthographe et de ponctuation. Cf. HeIss, Histoire de I'Empire (1685), t. III, p. 294; JACQUET, Recueil precieux, t. I, p. 404. 
Les paragraphes qui ne sont pas cités in extenso, sont très brièvement résumés ${ }^{431}$. Le paragraphe résumant ces clauses, inséré en lettres italiques avant la conclusion du traité, est d'une teneur identique dans les ouvrages de Heiss et de Jacquet ${ }^{432}$. En ce qui concerne la déclaration de Ferdinand Ier relative à cette paix (Declaratio Ferdinandea), on note également que Jacquet a repris la traduction française de Heiss $^{433}$. Il l'a insérée dans son »Droit public « en $1782^{434}$, et dans son »Recueil precieux en $1787^{435}$. Non seulement la traduction, mais aussi le titre que Jacquet a donné à ce document remontent à Heiss ${ }^{436}$. Comme ce dernier, Jacquet reproduit le texte de la déclaration de Ferdinand ler sans annotation ${ }^{437}$.

À ces traductions imprimées il faut ajouter les manuscrits. Le dépôt des Affaires étrangères possédait un certain nombre de mémoires traitant des problèmes religieux, par exemple un mémorandum intitulé »Des trois Relligions en Allemagne dont l'exercice est permis ou toléré«, daté du mois de mars 1741 , qui donne un catalogue de l'appartenance confessionnelle des villes et des territoires de l'Empire ${ }^{438}$; ce mémoire contient aussi des résumés très sommaires du traité de Passau, de $1552^{439}$, et de la paix de religion d'Augsbourg, de $1555^{440}$. Or, malgré ces traductions (moins nombreuses que celles des autres lois fondamentales), beaucoup d'auteurs français ne comprenaient pas bien le droit de religion allemand $^{441}$. Les documents français relatifs au droit de religion sont rares ${ }^{442}$.

431 Cf. HeIss, Histoire de l'Empire (1685), t. III, p. 304.

432 Cf. ibid.; JACQUET, Recueil precieux, t. I, p. 418.

433 Cf. HeIss, Histoire de l'Empire (1685), t. III, p. 306-308.

434 Cf. JACQUET, Droit public d'Allemagne, t. IV, p. 419-422.

435 Cf. ID., Recueil precieux, t. I, p. 419-422.

436 Cf., pour le titre, HeISS, Histoire de l'Empire (1685), t. III, p. 306; JACQUET, Recueil precieux, t. I, p. 419.

${ }^{437}$ Contrairement à Heiss, Jacquet finit avec la signature de Ferdinand Ier. Heiss a ajouté une mention omise par Jacquet, HeISs, Histoire de l'Empire (1685), t. III, p. 308. Ces contre-signatures qui y étaient évoquées ont, de toute évidence, été jugées inutiles par Jacquet.

438 Copie: AE, MD All. 12 fol. 184-190'; la datation a été ajoutée par une autre main que celle du copiste.

439 Ibid., fol. 186'-187: »Des édits de pacifications [sic] pour la relligion en Almagne [sic], Bohême et Silésie«, le premier paragraphe de cette partie du document concerne les règlements de 1552.

440 Ibid., fol. 187-187': "Édit de pacification d'Augsbourg l'an 1555«; le résumé précise que cette paix ne permet que l'exercice du catholicisme et celui de la confession d'Augsbourg, mentionnant aussi le Reservatum ecclesiasticum et le ius reformandi (sans employer ces termes techniques).

441 Beaucoup d'auteurs français n'avaient que des connaissances approximatives en matière de droit de religion. Certains confondaient même le traité de Passau (1552) avec la paix d'Augsbourg (1555), cf., par exemple, BNF, F.fr. 14619: "Mémoires sur l'Allemagne. Première partie « (titre, fol. [II]); note, fol. [I]: "L'auteur est monsieur Le Blanc connu par ses négotiations et par son traité des mon[n]oies«; ici p. [240]: „L'on n'avoit pas si bien réglé par le traitté de Passau fait en 1555, les différents de la religion en Allemagne, qu'ils [sic] ne reste encore aux catholiques et aux protestants des prétextes plausibles de se désunir par les diverses interprétations que chacun donnoit aux termes du traitté «. Mais l'auteur indique la date correcte de 1552, ibid., p. [183], et fait même preuve de connaissances solides à l'égard de certaines matières. Probablement, ce texte fut rédigé entre 1690 et 1710. 


\section{Conclusion}

L'inventaire de toutes les éditions des traités de Westphalie en langue française parues sous l'Ancien Régime révèle le nombre considérable d'au moins quinze éditions intégrales du traité d'Osnabriick et de vingt-six du traité de Münster entre 1651 et 1787, ainsi que trois résumés du traité d'Osnabrück, parus de 1648 à 1650 , et sept résumés du traité de Münster, parus durant la même période. Il faut y ajouter une réédition de l'abrégé de ce dernier traité datant de 1674, et il ne s'agit que des éditions qui sont encore conservées aujourd'hui. On peut conjecturer qu'au moins une autre édition intégrale du traité de Münster doit avoir vu le jour. Â ces éditions s'ajoutent des extraits publiés dans des recueils de documents officiels ou dans des ouvrages de jurisprudence. Sont conservées, de plus, plusieurs copies manuscrites de traductions ou de résumés ainsi que des imprimés annotés des traités de Westphalie, au Quai d'Orsay et dans plusieurs bibliothèques en France, comme à la bibliothèque municipale de Dijon, par exemple. La publication des traités de paix constituait, sous l'Ancien Régime, un véritable enjeu commercial. Ces éditions visaient en particulier, mais pas exclusivement, les hommes d'État, les négociateurs, les historiens et les publicistes. Elles s'adressaient également aux hommes cultivés en général. En ce qui concerne la forme de gouvernement du Saint-Empire, les idées divergentes des historiens et des juristes qui expliquent ces traités ne sont pas liées à l'emploi d'un vocabulaire spécifique. Les idées ne sont pas attachées aux mots, mais aux notions.

Après la paix de Westphalie, la Bulle d'or fut la loi fondamentale du Saint-Empire, avec le plus grand nombre de traductions françaises aux XVIIe et XVIIIe siècles: vingt-sept traductions intégrales et deux abrégés publiés entre 1612 et 1787. Mais en additionnant toutes les capitulations impériales qui furent traduites et publiées en français, on arrive à un chiffre encore plus considérable: cinquante traductions publiées également de 1612 à 1787 , dont deux abrégés. La capitulation de Charles VI arrive en tête avec douze publications; celle de Léopold Ier fut publiée onze fois; pour Joseph Ier, il y en a sept; six pour Charles Quint (dont deux abrégés); cinq pour François Ier; quatre pour Ferdinand III; trois pour Charles VII;

442 Cependant, il y a des exceptions considérables; cf., par exemple, le volume AE, MD All. 71 , qui contient plusieurs notes relatives au $»$ Droit que les Allemands appellent jus eundi in partes « (»Mémoire sur le droit des états de l'Empire de se séparer en deux parties, à l'effet d'opérer que la pluralité de suffrages ne puisse pas avoir lieu«); ces notes sont datées de 1757 et de 1759 (AE, MD All. 71 fol. 142-147 [première citation fol. 142], fol. 276-282, 283284', 285-293 et 294-302 [deuxième citation fol. 294]). Le volume contient également un "Essai d'interprétation « de l' article V de la paix d'Osnabrück (cet article concerne le droit de religion), daté de 1759 (ibid., fol. 303-307), et une note intitulée »Les principes que monsieur Pfeffel établit dans son mémoire sur le droit de réformer des états de l'Empire « du mois d'octobre 1759 (ibid., fol. 328-330). Cf. aussi la »Dissertation sur l'uniformité des suffrages requise dans les diètes de l'Empire«, en particulier »dans les causes de religion«, de 1733 (AE, MD All. 74 fol. 155-224). En outre, le volume AE, MD All. 8 (1521-1620) contient des traductions de déclarations impériales relatives au droit de religion (par exemple, l'édit de Charles Quint de 1521 et le fameux édit de Restitution de Ferdinand II de 1629). 
enfin, deux pour Joseph II. À cela s'ajoute la publication synoptique de toutes les capitulations des souverains des XVIe et XVIIe siècles, qui comprend aussi la capitulation de Ferdinand IV, roi des Romains mort avant d'accéder à la tête de l'Empire. Parmi les autres lois de l'Empire, on note deux traductions de la paix publique de 1548; deux de l'ordonnance d'exécution de 1555; deux du recès de 1654 , qui était le dernier recès de l'Empire, puisque la diète de Ratisbonne s'éternisa sans pouvoir voter de recès jusqu'à la dissolution du Saint-Empire à l'aube du XIX ${ }^{\mathrm{e}}$ siècle. Enfin, on compte trois traductions de projets pour une capitulation impériale perpétuelle, qui était un sujet souvent débattu par la diète de l'Empire depuis la deuxième moitié du XVIIe siècle, sans qu'elle soit capable de voter l'un des projets discutés.

En ce qui concerne les lois fondamentales en général, les publications de la période antérieure à 1648 sont peu nombreuses: quatre abrégés. Dans la période qui va de 1649 à 1699 , la donne change déjà considérablement: à cette époque, on repère au moins dix-neuf traductions intégrales. Ce chiffre ne cesse de croître au XVIII' siècle: cinquante et une traductions intégrales pour la période 1700-1739. En l'espace de dix-sept ans, de 1740 à 1756, on compte trente traductions intégrales. Enfin, pour la fin de l'Ancien Régime, entre 1757 et 1789, on dénombre encore dix-sept traductions intégrales. Dans ce contexte, il faut aussi souligner que les documents publiés au XVIII e siècle, notamment les capitulations impériales, étaient souvent beaucoup plus longs que les lois publiées précédemment, et que très souvent les auteurs accompagnaient leurs traductions d'un commentaire très détaillé. En règle générale, le travail des traducteurs a été d'une très grande qualité, malgré quelques ambiguités et certaines erreurs mineures, qui ne manquent presque jamais dans toutes les traductions. En ce qui concerne la terminologie, nous pouvons dresser le même constat que pour les traductions des traités de paix de Westphalie: encore chancelante avant 1648 , la terminologie française relative au droit public allemand est définitivement forgée dans la seconde moitié du XVIIe siècle. Depuis ce temps-là, non seulement les traducteurs, mais aussi les juristes, les historiens, les géographes et les diplomates qui décrivent le Saint-Empire en langue française utilisent les mêmes termes que les historiens de nos jours. Au XVIII e siècle, un certain nombre de termes techniques, qui sont hors d'usage à l'heure qu'il est, même pour les spécialistes du Saint-Empire, ont été introduits dans les traductions françaises des lois fondamentales et dans le langage des juristes et des historiens.

La Bulle d'or et les capitulations impériales furent souvent traduites à toutes les époques que nous avons distinguées dans le paragraphe précédent. En revanche, les lois fondamentales relatives au droit religieux de l'Empire ne suscitèrent l'intérêt des traducteurs que dans la deuxième moitié du XVIIe siècle: avant l'»Histoire de l'Empire« de Jean Heiss (1684), leurs traductions intégrales semblent faire défaut, quoiqu'elles datent du XVIe siècle et que la guerre de Trente Ans ait aussi été une guerre de religion. $A$ contrario, tout au long de la période étudiée, de la veille de la guerre de Trente Ans à la Révolution française, on assiste à une diversification constante. Même dans les années 1780 , on continue à s'intéresser 
aux plus anciennes lois fondamentales de l'Empire (la Bulle d'or et les lois relatives au droit de religion du $\mathrm{XVI}^{e}$ siècle), dont on publie des traductions à côté des capitulations impériales récentes. Cependant, dans la période suivant la mort de Charles VI, durant laquelle le trône impérial échappa temporairement à la maison d'Autriche, posant le problème de l'élection impériale au cœur des politiques française, allemande et européenne, les publications de la Bulle d'or et des capitulations des empereurs furent particulièrement nombreuses: huit éditions de la Bulle d'or, dix-neuf éditions de capitulations impériales (dont la grande édition synoptique de toutes les capitulations des XVIe et XVIIe siècles que nous n'avons comptée que comme une seule publication) et trois éditions de projets pour la capitulation perpétuelle. À cette époque, qui va de la mort de Charles VI à l'éclatement de la guerre de Sept Ans, nous n'avons pas retrouvé d'éditions françaises des autres lois fondamentales du Saint-Empire (à l'exception de la paix de Westphalie), c'est-à-dire que, de toute évidence, l'attention du public francophone était entièrement focalisée sur le droit de l'élection impériale.

Le rythme des élections s'accélère, avec un pic de la production de traductions françaises durant la période 1741-1756. Nous n'avons pas trouvé d'éditions françaises pour les années $1613-1632,1634-1657,1659-1683,1686-1688,1690$ $1693,1695-1704,1706-1710,1712-1714,1716-1727,1729-1730,1732,1734$ $1740,1744,1747,1751-1753,1755,1757-1774,1776-1781,1783-1786$ et 1788-1789443. De la veille de la guerre de Trente Ans à l'éclatement de la guerre de Sept Ans, le nombre d'années sans publications françaises des lois fondamentales de l'Empire diminue donc, alors que les intervalles redeviennent plus importants après 1756. L'année avec le plus grand nombre d'éditions est 1711: au total, douze éditions, dont six traductions de la Bulle d'or. Ce chiffre s'explique par la mort de l'empereur Joseph $\mathrm{I}^{\mathrm{er}}$ et l'avènement de Charles $\mathrm{VI}^{444}$. Outre les intervalles, le délai entre la promulgation d'une loi fondamentale et sa publication en français tendait à diminuer avant 1756 . En 1658 et au moment des élections impériales ultérieures, les auteurs et les éditeurs proposèrent à leurs lecteurs des éditions des capitulations des empereurs morts avant l'élection qui était à l'ordre du jour. En 1658, on publia la capitulation de Ferdinand III, élu en 1636. En revanche, selon nos recherches, la capitulation de Léopold Ier, élu en 1658, ne fut publiée en français qu'en 1684. En 1711, la capitulation la plus récente qui est alors publiée est celle de Joseph Ier (1690). La capitulation de Charles VI, élu en 1711, sera publiée en français en 1715 (à La Haye), sa deuxième édition date de 1728 (c'est la première publiée en France). A contrario, celle de Charles VII est traduite en français dès l'année de son élection (1742); celle de François $\mathrm{I}^{\text {er }}$ est donnée au public dans l'année suivant son élection (c'est-à-dire en 1746), et encore faut-il

443 Ont été prises en compte les années 1612 (publications de Canini et Le Secq) à 1789 (Révolution française).

444 Même chiffre de douze publications annuelles en 1731 et en 1733, mais à cette époque, le nombre considérable d'éditions s'explique par la publication de plusieurs éditions de l'»Histoire de l'Empire « de Jean Heiss, dont chacune contient six lois fondamentales depuis l'adjonction de la capitulation de Charles VI à partir de 1715 . 
prendre en considération qu'il fut élu en septembre 1745 , alors que son prédécesseur l'avait été au mois de janvier 1742 . On constate donc à la fois une intensification et une actualisation des publications françaises. Finalement, il faut aussi noter que des résumés ou des extraits de lois fondamentales furent publiés dans certains ouvrages de droit et d'histoire, et que les fonds d'archives contiennent des traductions françaises partielles, notamment des capitulations impériales ${ }^{445}$. Leur existence accentue encore le résultat de notre analyse des traductions imprimées, qui a démontré que les versions françaises des lois fondamentales du SaintEmpire sont beaucoup plus nombreuses qu'on ne l'a supposé. Parfois, on peut saisir l'utilisation de ces traductions par les hommes politiques ${ }^{446}$. Plusieurs traducteurs se sont soumis à la tâche difficile qui consistait à traduire ces lois: des Français et des étrangers, en particulier des Allemands et des Alsaciens. Mais cela n'ôte rien au mérite qui revient à Jean Heiss en tant que principal traducteur des lois fondamentales du Saint Empire romain germanique. À partir de la publication du "Discours Historique « d'Abraham de Wicquefort en 1658, les lecteurs français disposèrent des premières bonnes traductions de la Bulle d'or et de la capitulation de Ferdinand III, mais pour les autres lois fondamentales de l'Empire germanique, il fallut encore attendre l'»Histoire de l'Empire « de Heiss en 1684.

445 On publie même des éditions partielles fondées sur d'autres éditions partielles plus complètes. Cf., par exemple, Boug D'ORSCHWILLER, Ordonnances d'Alsace, t. I, p. XXXVIXXXVIIl („Extrait De La Transaction conclue \& ratifiée à Passau sous l'autorité de Charles Quint, Empereur, toujours auguste, entre Ferdinand, Sérénissime Roi des Romains, \&c. \& quelques Etats d'Allemagne « [1552]: extraits et résumés du préambule et des chapitres I-III; cette traduction partielle correspond en principe littéralement à celle qui est publiée dans l'»Histoire de l'Empire « de Heiss, mais sans les notes de bas de page, et parfois, on note des différences mineures: par exemple, Boug, p. XXXVI, écrit »paternellement«, au préambule du traité, tandis que Heiss a la leçon correcte: »fraternellement«, cf. HeIss, Histoire de l'Empire [1733, édition in- $8^{\circ}$ ], t. VI, p. 482 [= t. II, p. 388 dans l'édition in- $4^{\circ}$ ]). Comme, en règle générale, ces éditions et ces copies partielles ne sont pas de nouvelles traductions, mais reproduisent des versions déjà connues, il ne serait pas utile d'en dresser une liste exhaustive. 446 En 1699, pour justifier le terme »prétendus reformez « dans la liste des lieux compris dans l'article IV de la paix de Ryswick, Louis XIV prétendait que la même expression avait été utilisée dans la capitulation impériale de 1658 . Or, Chamoy répondit à Torcy que cette expression n'était employée que dans la version française de cette capitulation; cf. Anna SINKOLI, Frankreich, das Reich und die Reichsstände 1697-1702, Francfort/M. et al. 1995 (Europäische Hochschulschriften, série III: Geschichte und ihre Hilfswissenschaften, 652), p. $165-166$. 
